\title{
Sulfur-Doped (Dibenzo)heptazethrene and (Dibenzo)octazethrene Diradicaloids
}

\author{
Chi Hao Eugene Chow ${ }^{\dagger}$ Hoa Phan, ${ }^{\dagger}$ Xiaojie Zhang, ${ }^{*}, J^{\ddagger}$ Jishan $\mathrm{Wu}^{*, \dagger, \perp}$ \\ 'Department of Chemistry, National University of Singapore, 3 Science Drive 3, 117543, \\ Singapore \\ Hebei Key Laboratory of Functional Polymers, Department of Polymer Materials and \\ Engineering, Hebei University of Technology, 8 Guangrong Street, Tianjin 300130, P. R. China \\ ${ }^{\perp}$ Joint School of National University of Singapore and Tianjin University, International \\ Campus of Tianjin University, Binhai New City, Fuzhou 350207, P. R. China
}

\section{Table of contents}

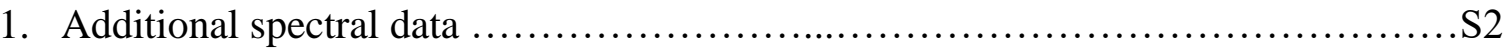

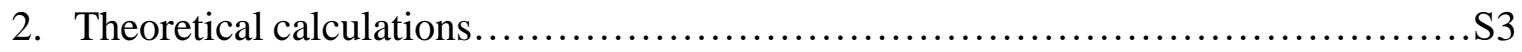

3. NMR and mass spectra of new compounds ...................................... 6

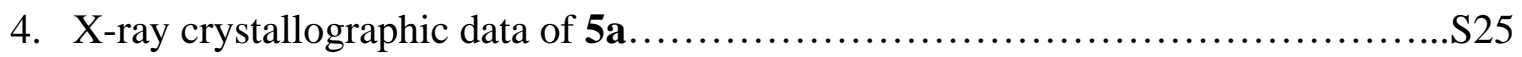

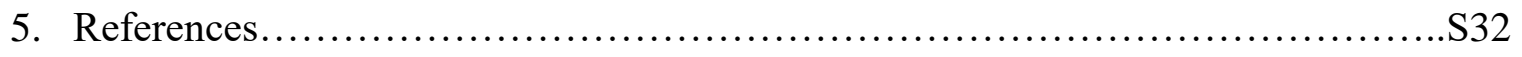

6. Appendix: Cartesian coordinates and other computational results................S33 
1. Additional spectral data
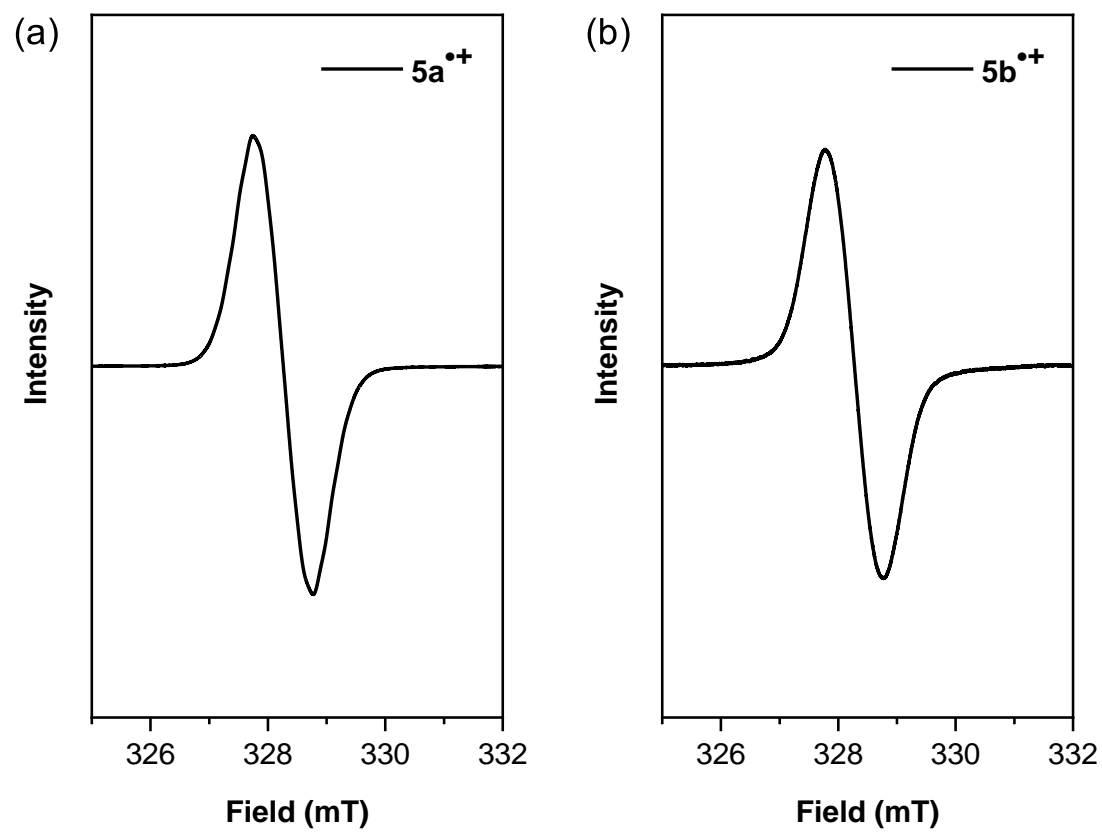

Figure S1.ESR spectra of (a) $\mathbf{5 a}^{\cdot+}$ and (b) $\mathbf{5} \mathbf{b}^{\mathbf{}}$ in $\mathrm{CH}_{2} \mathrm{Cl}_{2}$ solution at room temperature.
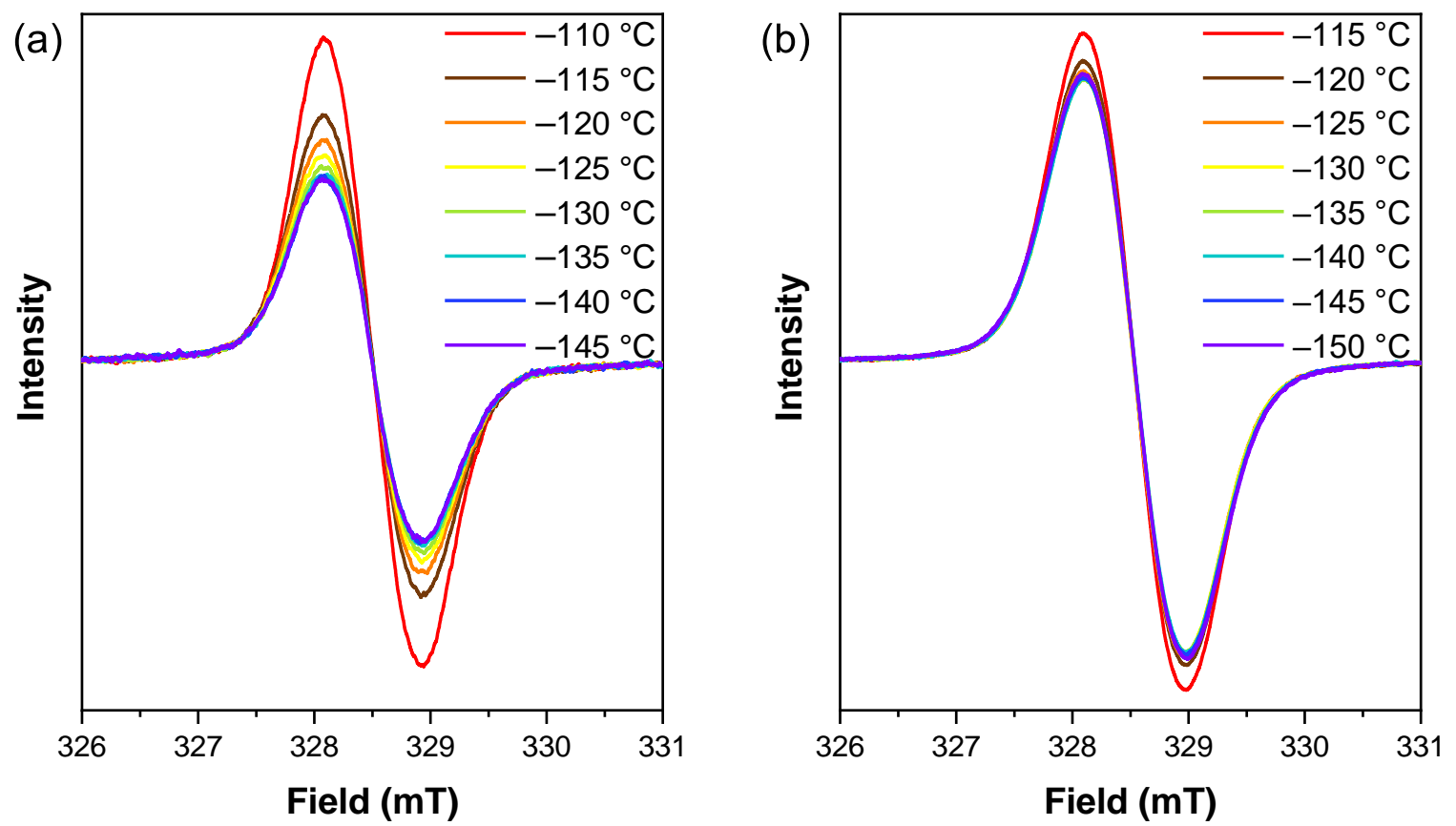

Figure S2. VT ESR spectra of (a) DBHZ-2S and (b) DBOZ-2S in frozen $\mathrm{CH}_{2} \mathrm{Cl}_{2}$ solution. 


\section{Theoretical calculations}

Theoretical calculations were performed with the Gaussian09 rev. D program suite. ${ }^{1}$ All calculations were carried out using the density functional theory (DFT) method with Becke's three-parameter hybrid exchange functionals and the Lee-Yang-Parr correlation functional (B3LYP) employing the 6-31G(d,p) basis set for all atoms. ${ }^{2}$ NOON (natural orbital occupation number) calculations were done by spin unrestricted UCAM-B3LYP/6-31G(d,p) method and the diradical character $\left(y_{0}\right)$ was calculated according to Yamaguchi's scheme: $y_{0}=1-(2 T /(1$ $\left.+T^{2}\right)$ ), and $T=\left(n\right.$ номо $\left.-n_{\text {LUMO }}\right) / 2$ ( $n$ номо is the occupation number $n$ of the HOMO, $n_{\text {LUMO is }}$ the occupation number $n$ of the LUMO). ${ }^{3}$ Time-dependent (TD) DFT calculations were done based on RB3LYP/6-31G(d,p) with CPCM as solvation method.

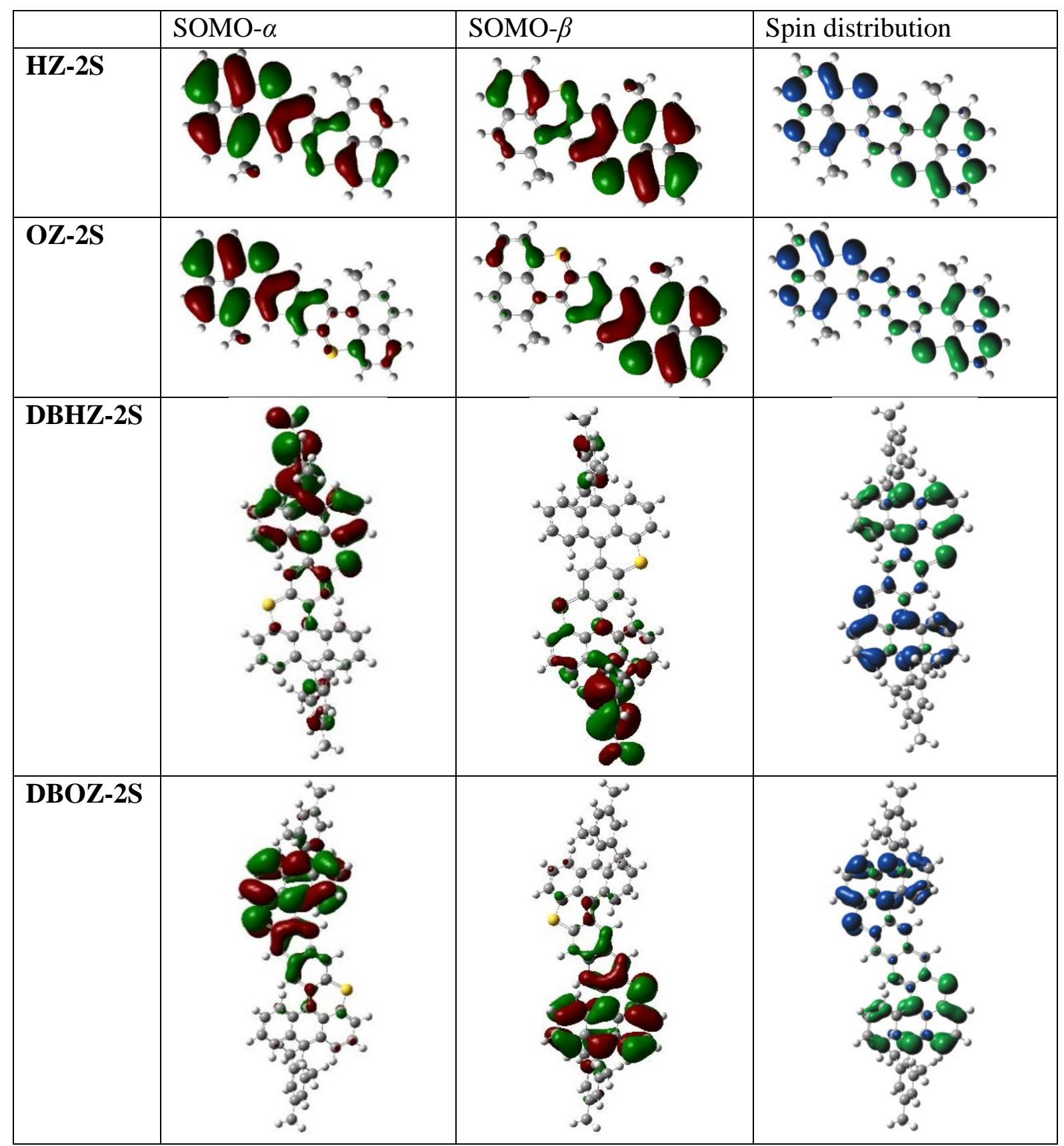

Figure S3. Calculated (UCAM-B3LYP/6-31G(d,p)) SOMO- $\alpha$ and SOMO- $\beta$ profiles and spin density distribution maps ( $\alpha$ spin $-\beta$ spin) for HZ-2S, OZ-2S, DBHZ-2S and DBOZ-2S. 


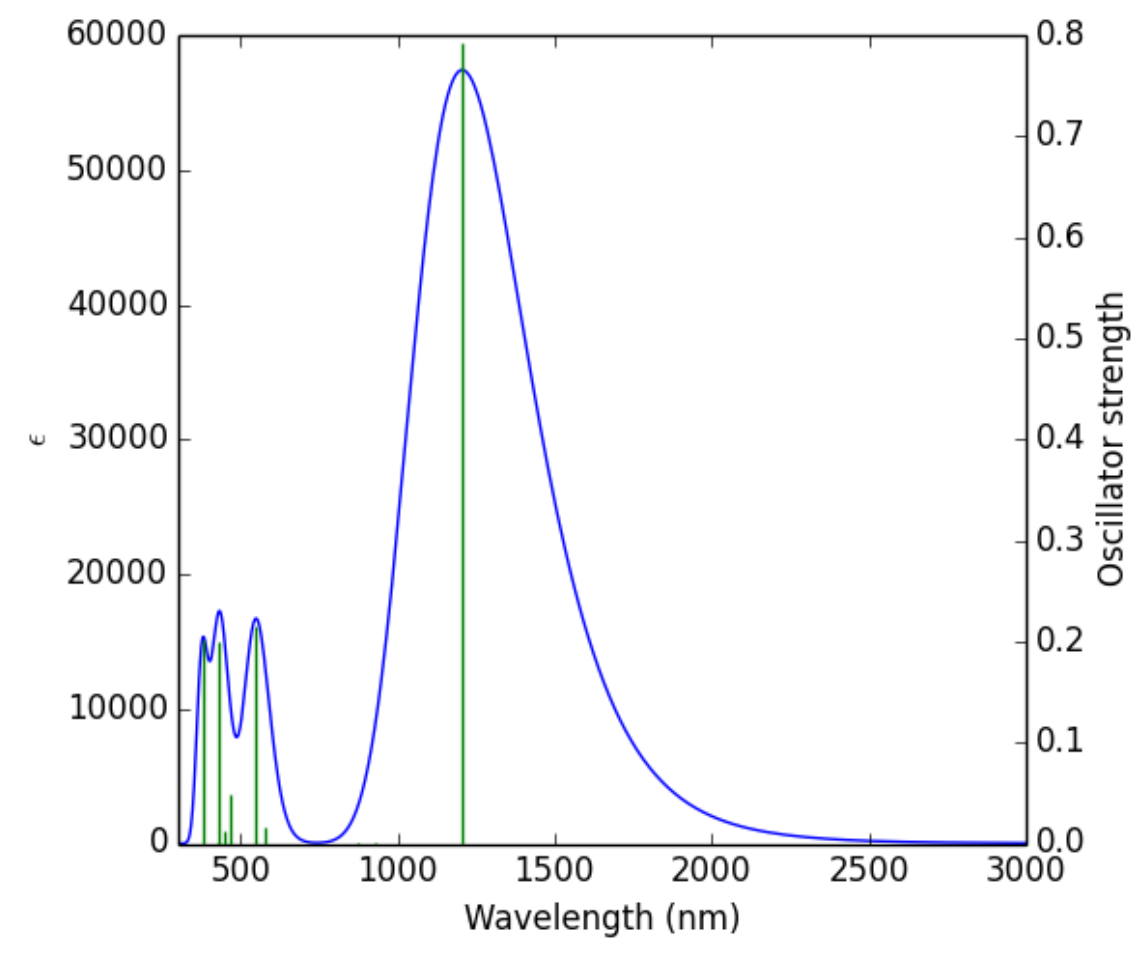

Figure S4. TD DFT simulated spectrum of DBHZ-2S.

Table S1. Selected TD-DFT (rB3LYP/6-31G) calculated energies, oscillator strength and compositions of major electronic transitions of DBHZ-2S.

\begin{tabular}{|l|l|l|}
\hline Wavelength $(\mathrm{nm})$ & Oscillator strength & Major contribution \\
\hline 379.3 & 0.2014 & H-1 $\rightarrow$ LUMO $(96 \%)$ \\
\hline 431.6 & 0.2004 & H-5 $\rightarrow$ L+1 $(95 \%)$ \\
\hline 549.2 & 0.2145 & $\begin{array}{l}\text { H-6 } \rightarrow \text { LUMO }(87 \%) \\
\text { H-8 } \rightarrow \text { LUMO }(9 \%)\end{array}$ \\
\hline 1205.5 & & HOMO $\rightarrow$ LUMO $(100 \%)$ \\
\hline
\end{tabular}




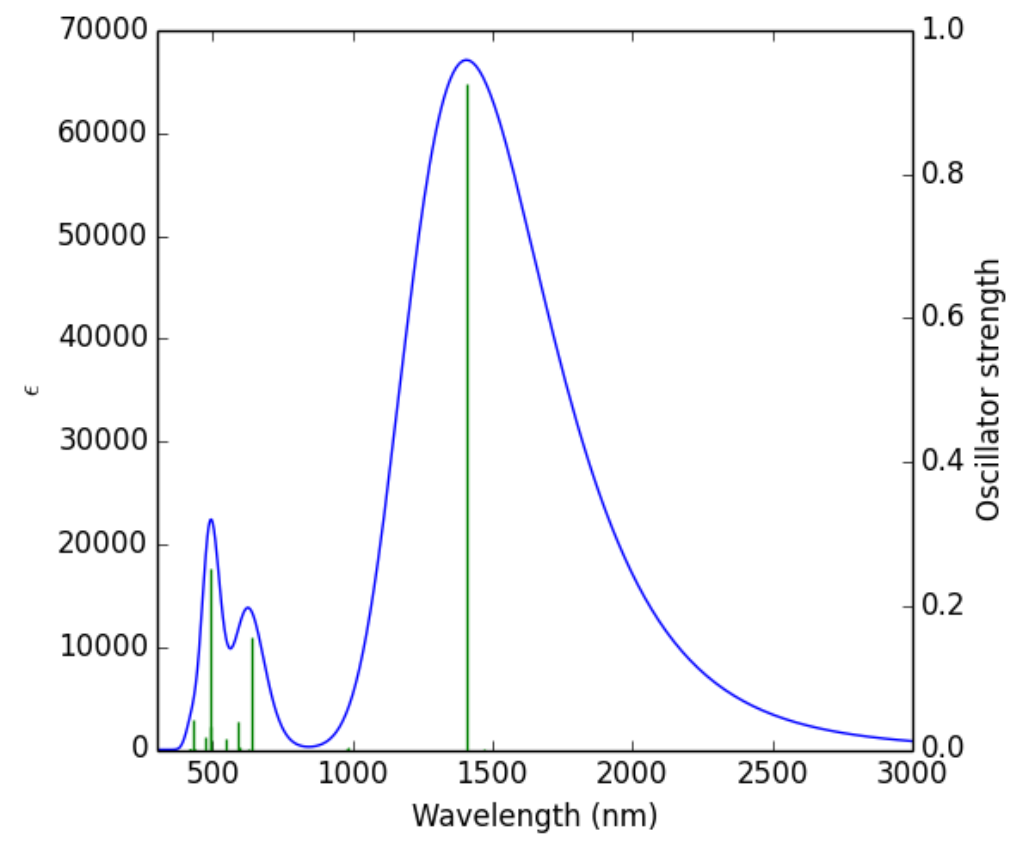

Figure S5. TD DFT simulated spectrum of DBOZ-2S.

Table S2. Selected TD-DFT (rB3LYP/6-31G) calculated energies, oscillator strength and compositions of major electronic transitions of DBOZ-2S.

\begin{tabular}{|l|l|l|}
\hline Wavelength $(\mathrm{nm})$ & Oscillator strength & Major contribution \\
\hline 495.1 & 0.2514 & $\begin{array}{l}\text { H-1 } \rightarrow \text { L+1 }(88 \%) \\
\text { HOMO } \rightarrow \text { L+2 }(10 \%)\end{array}$ \\
\hline 636.9 & 0.1561 & $\begin{array}{l}\text { H-8 } \rightarrow \text { LUMO }(10 \%) \\
\text { H-6 } \rightarrow \text { LUMO }(88 \%)\end{array}$ \\
\hline 1406.5 & 0.9248 & HOMO $\rightarrow$ LUMO $(100 \%)$ \\
\hline
\end{tabular}


3. NMR and mass spectra of new compounds

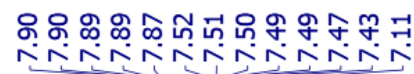

우쇾

Nํ
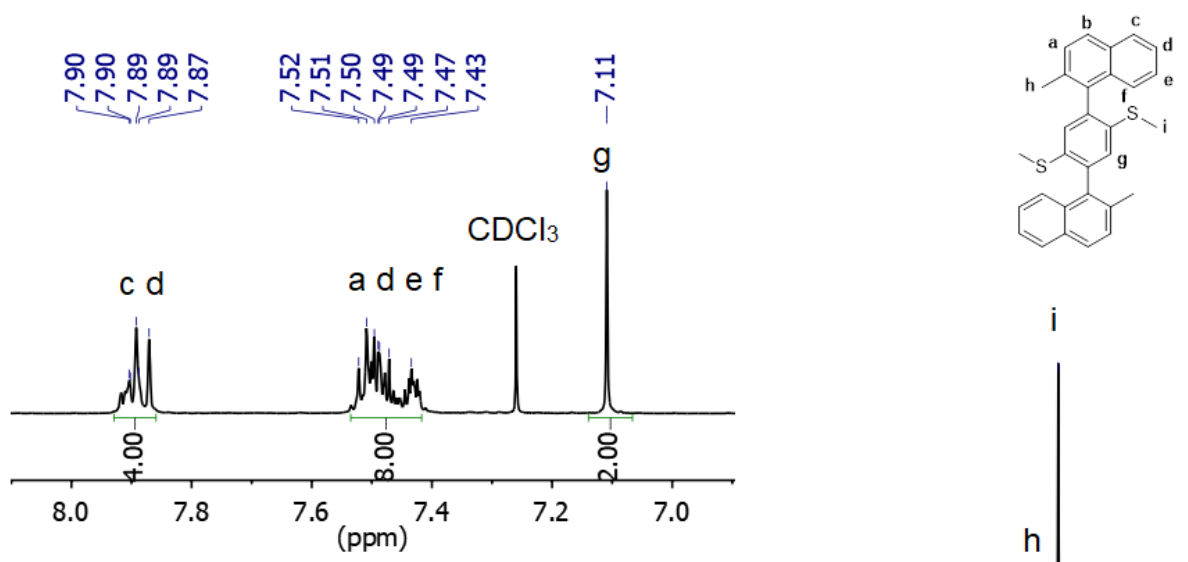

i

h

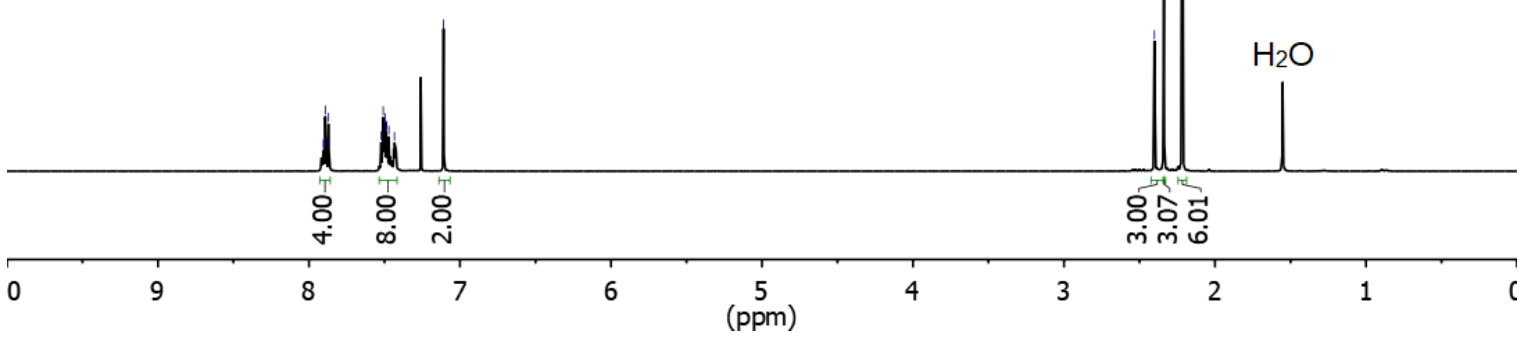

Figure S6. ${ }^{1} \mathrm{H}$ NMR spectrum of $\mathbf{3 a}\left(400 \mathrm{MHz}, \mathrm{CDCl}_{3}, \mathrm{rt}\right)$.

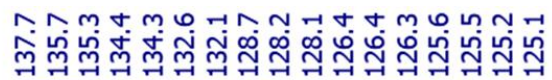

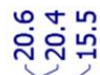

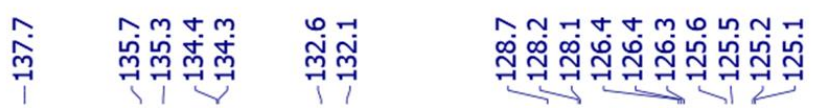

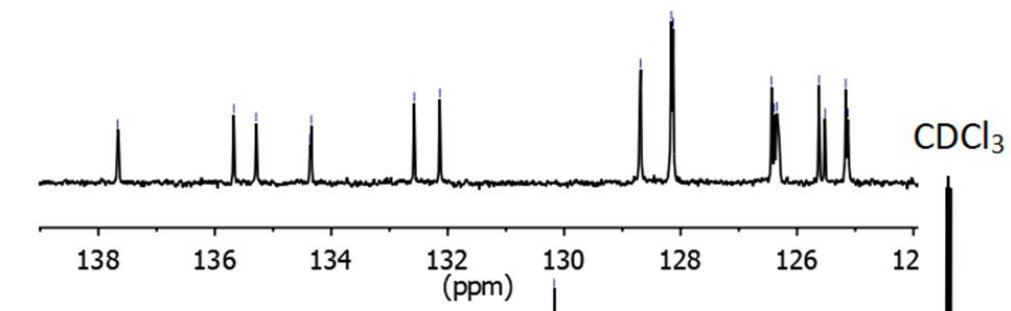

$\mathrm{CDCl}_{3}$

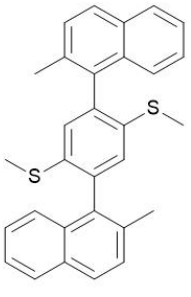

\begin{tabular}{lllll}
\hline 10 & 180 & 160 & 140 & 120
\end{tabular}

100
$(\mathrm{ppm})$

80

40

20

Figure S7. ${ }^{13} \mathrm{C}\left\{{ }^{1} \mathrm{H}\right\}$ NMR spectrum of $\mathbf{3 a}\left(500 \mathrm{MHz}, \mathrm{CDCl}_{3}, \mathrm{rt}\right)$. 

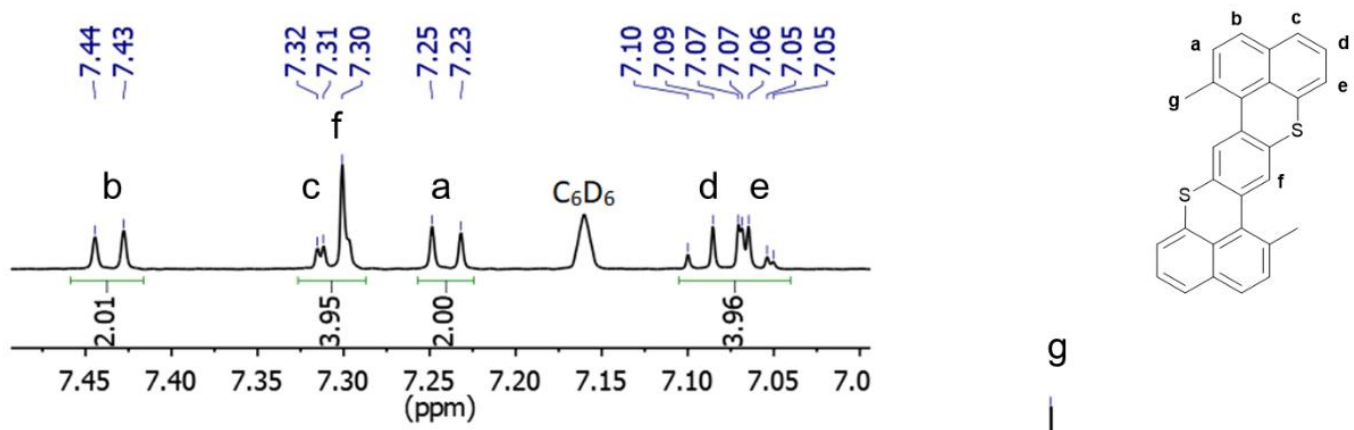

g

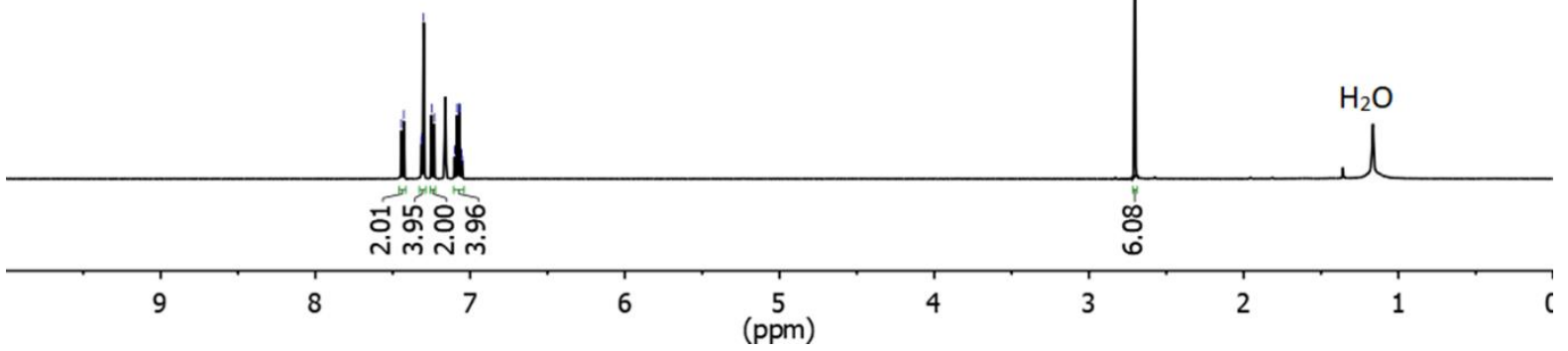

Figure S8. ${ }^{1} \mathrm{H}$ NMR spectrum of $5 \mathbf{a}\left(500 \mathrm{MHz}, \mathrm{CS}_{2} / \mathrm{C}_{6} \mathrm{D}_{6}, \mathrm{rt}\right)$.

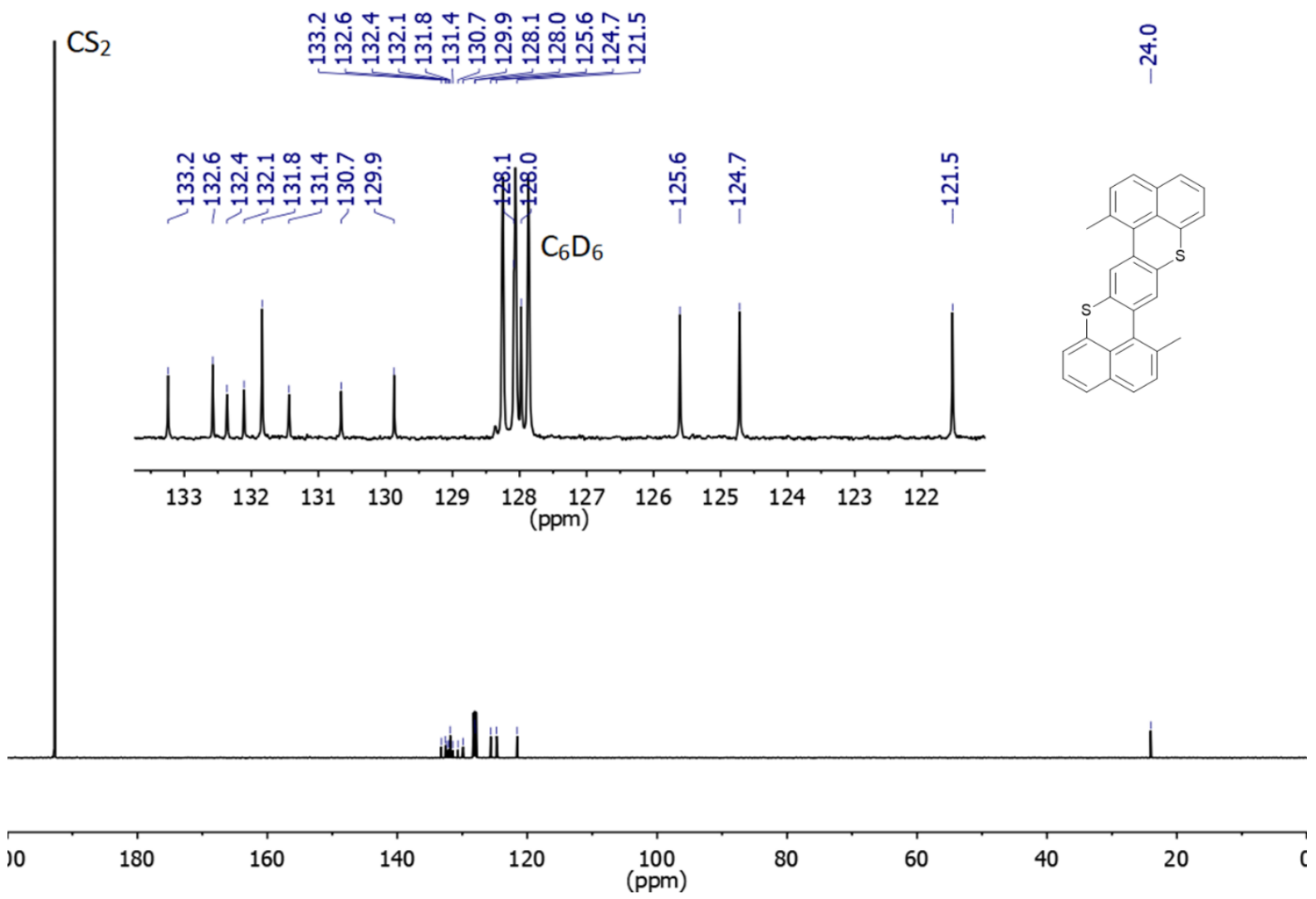

Figure S9. ${ }^{13} \mathrm{C}\left\{{ }^{1} \mathrm{H}\right\}$ NMR spectrum of $\mathbf{5 a}\left(500 \mathrm{MHz}, \mathrm{CS}_{2} / \mathrm{C}_{6} \mathrm{D}_{6}, \mathrm{rt}\right)$. 


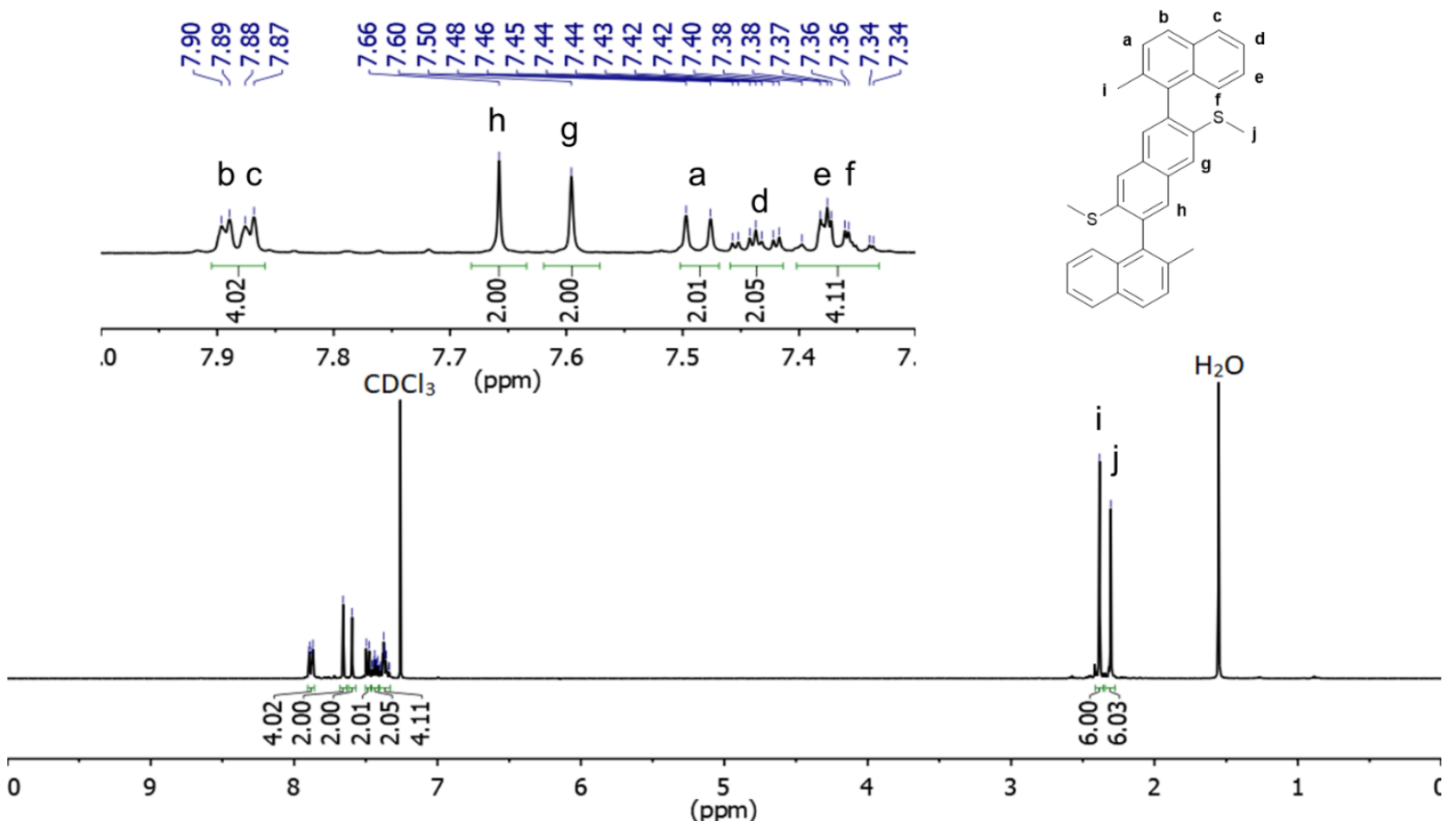

Figure S10. ${ }^{1} \mathrm{H}$ NMR spectrum of $\mathbf{3 b}\left(400 \mathrm{MHz}, \mathrm{CDCl}_{3}, \mathrm{rt}\right)$.

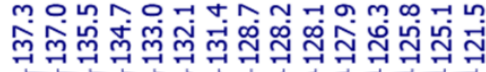

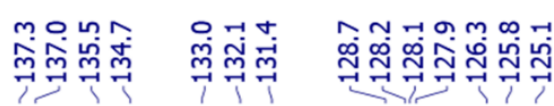

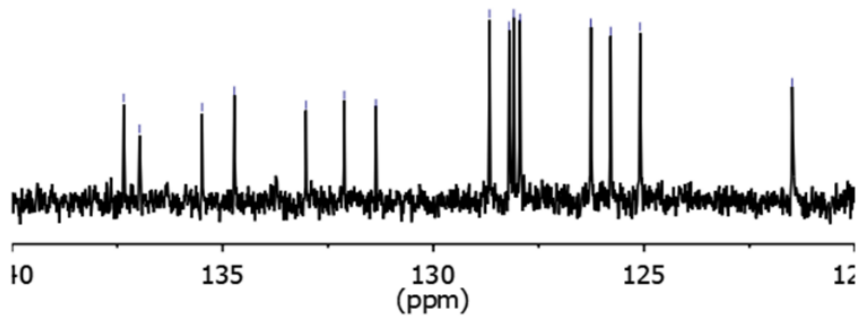

$\mathrm{CDCl}_{3}$

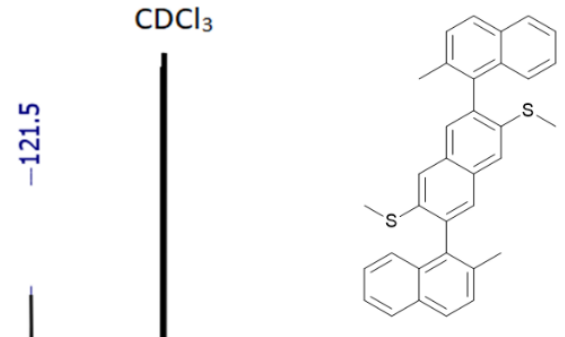

10190

Figure S11. ${ }^{13} \mathrm{C}\left\{{ }^{1} \mathrm{H}\right\}$ NMR spectrum of $\mathbf{3 b}\left(500 \mathrm{MHz}, \mathrm{CDCl}_{3}, \mathrm{rt}\right)$. 


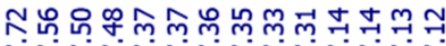

NNNNNNNRNR

$\stackrel{\substack{\infty \\ \text { i }}}{ }$
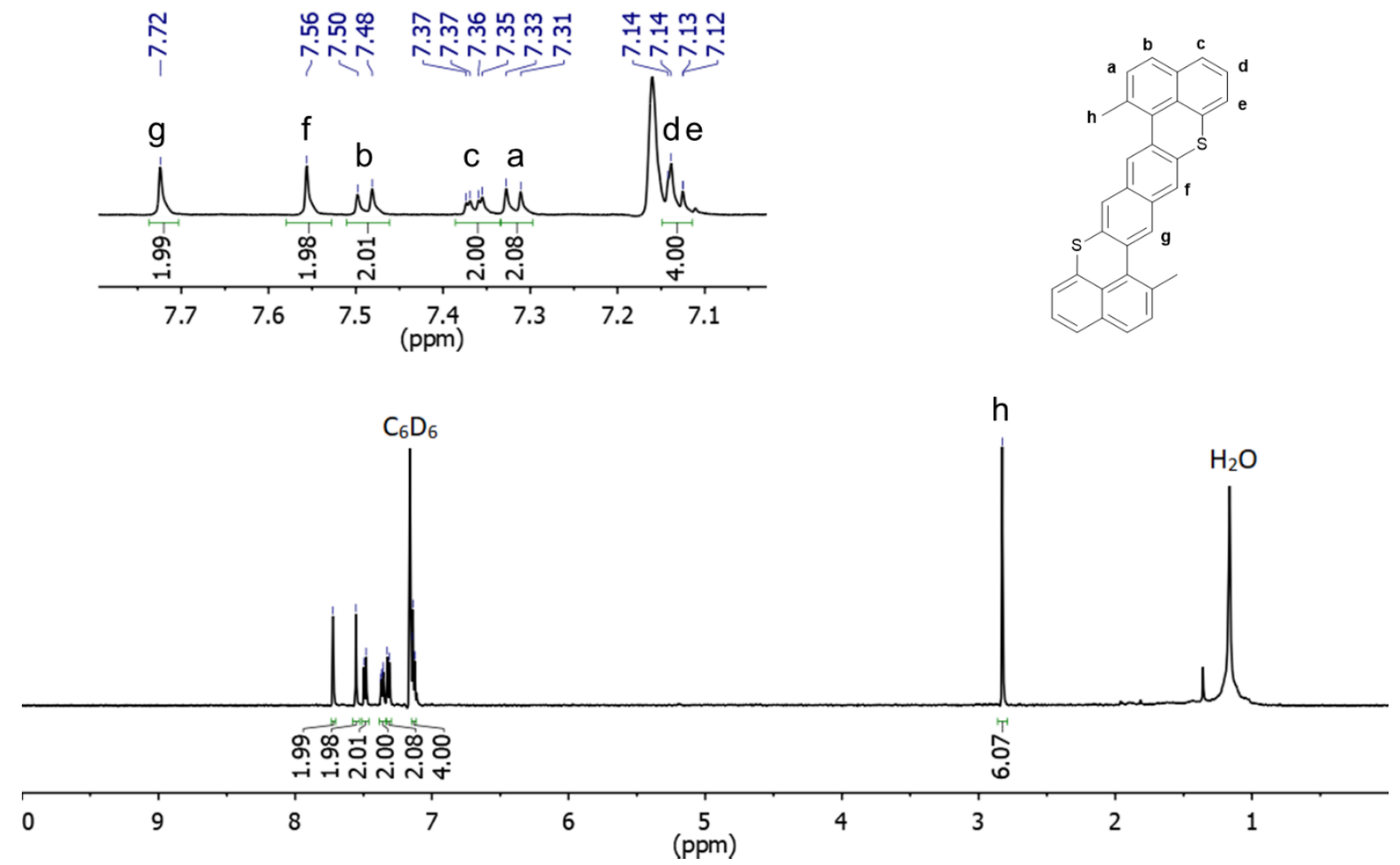

Figure S12. ${ }^{1} \mathrm{H}$ NMR spectrum of $\mathbf{5 b}\left(500 \mathrm{MHz}, \mathrm{CS}_{2} / \mathrm{C}_{6} \mathrm{D}_{6}, \mathrm{rt}\right)$.

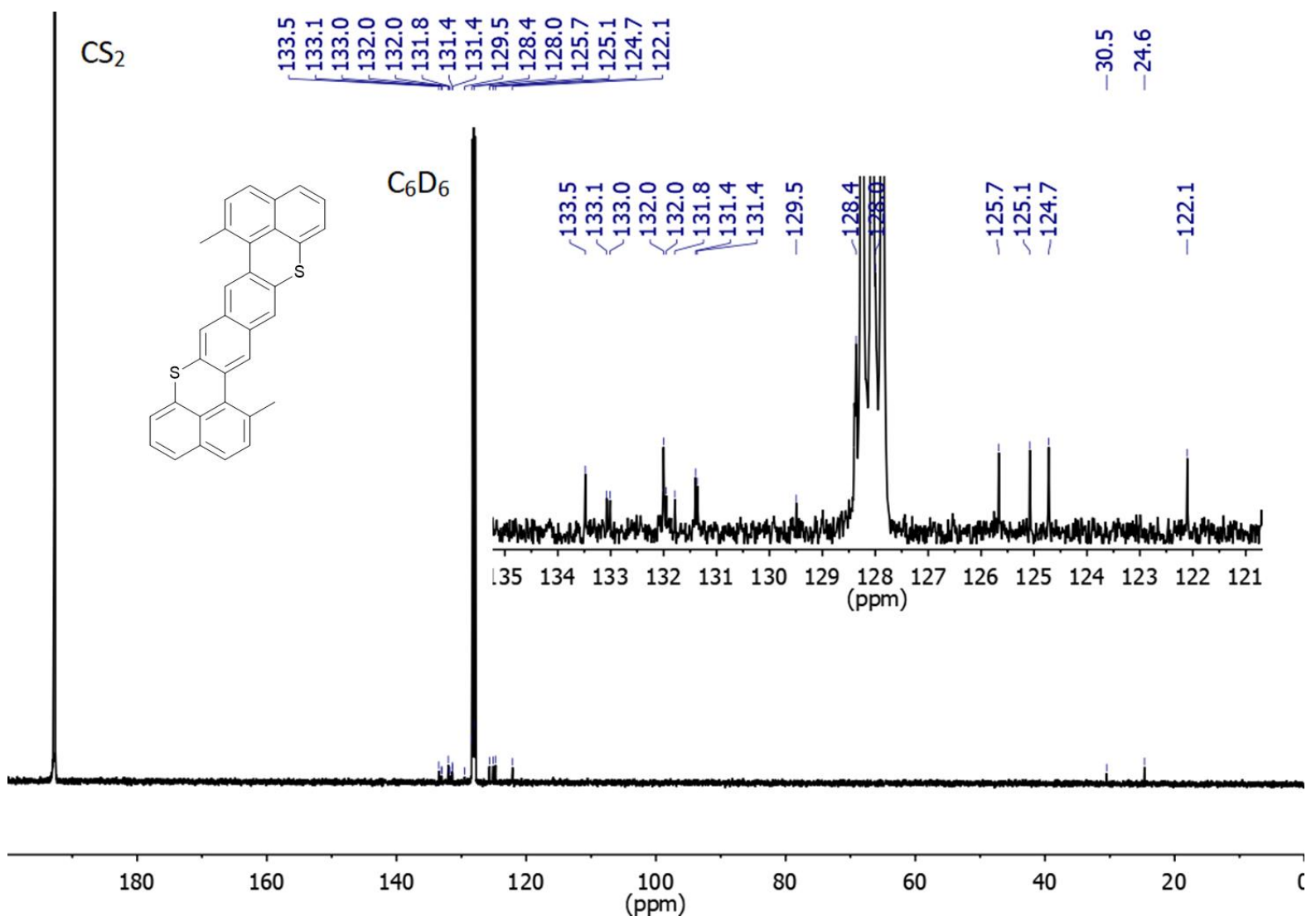

Figure S13. ${ }^{13} \mathrm{C}\left\{{ }^{1} \mathrm{H}\right\}$ NMR spectrum of $\mathbf{5 b}\left(500 \mathrm{MHz}, \mathrm{CS}_{2} / \mathrm{C}_{6} \mathrm{D}_{6}, \mathrm{rt}\right)$. 

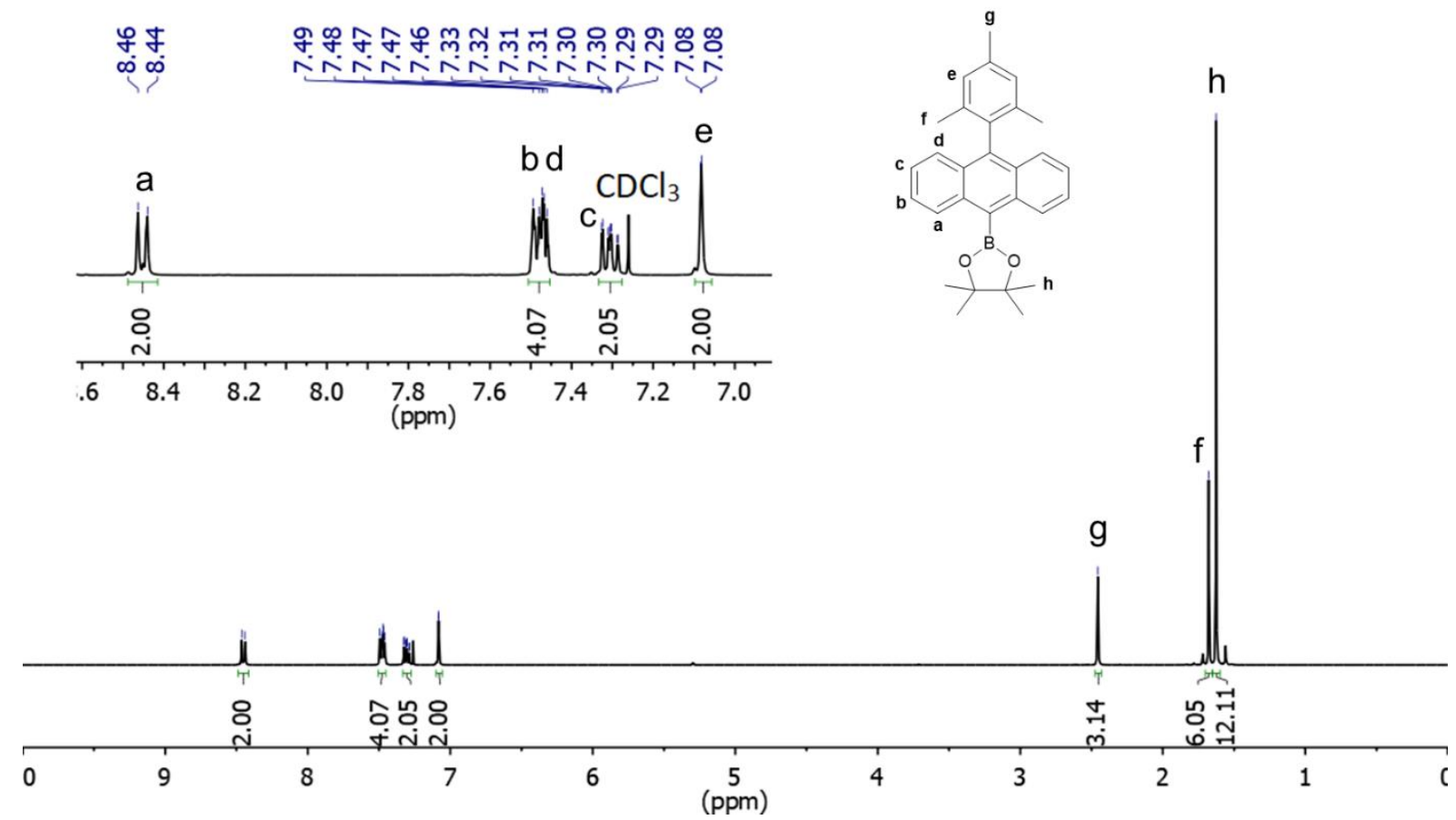

Figure S14. ${ }^{1} \mathrm{H} \mathrm{NMR}$ spectrum of $6\left(400 \mathrm{MHz}, \mathrm{CDCl}_{3}, \mathrm{rt}\right)$.
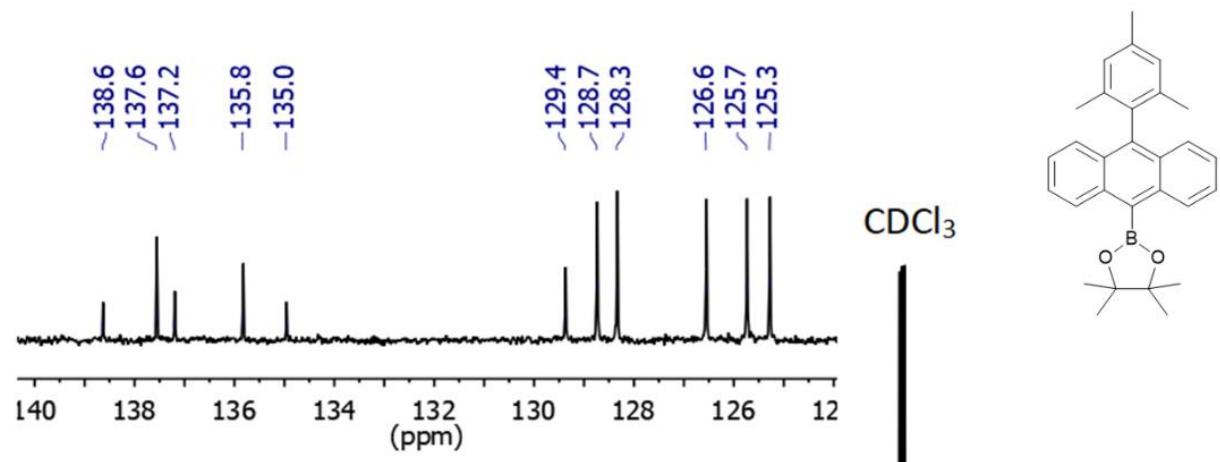

$$
140
$$

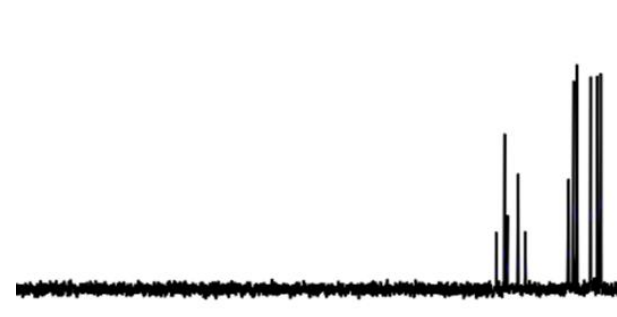

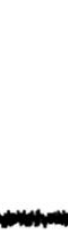

180

$180 \quad 160$

160

140

120

100
$(\mathrm{ppm})$

80

60

60

40

$20 \quad c$

Figure S15. ${ }^{13} \mathrm{C}\left\{{ }^{1} \mathrm{H}\right\}$ NMR spectrum of $6\left(400 \mathrm{MHz}, \mathrm{CDCl}_{3}, \mathrm{rt}\right)$. 

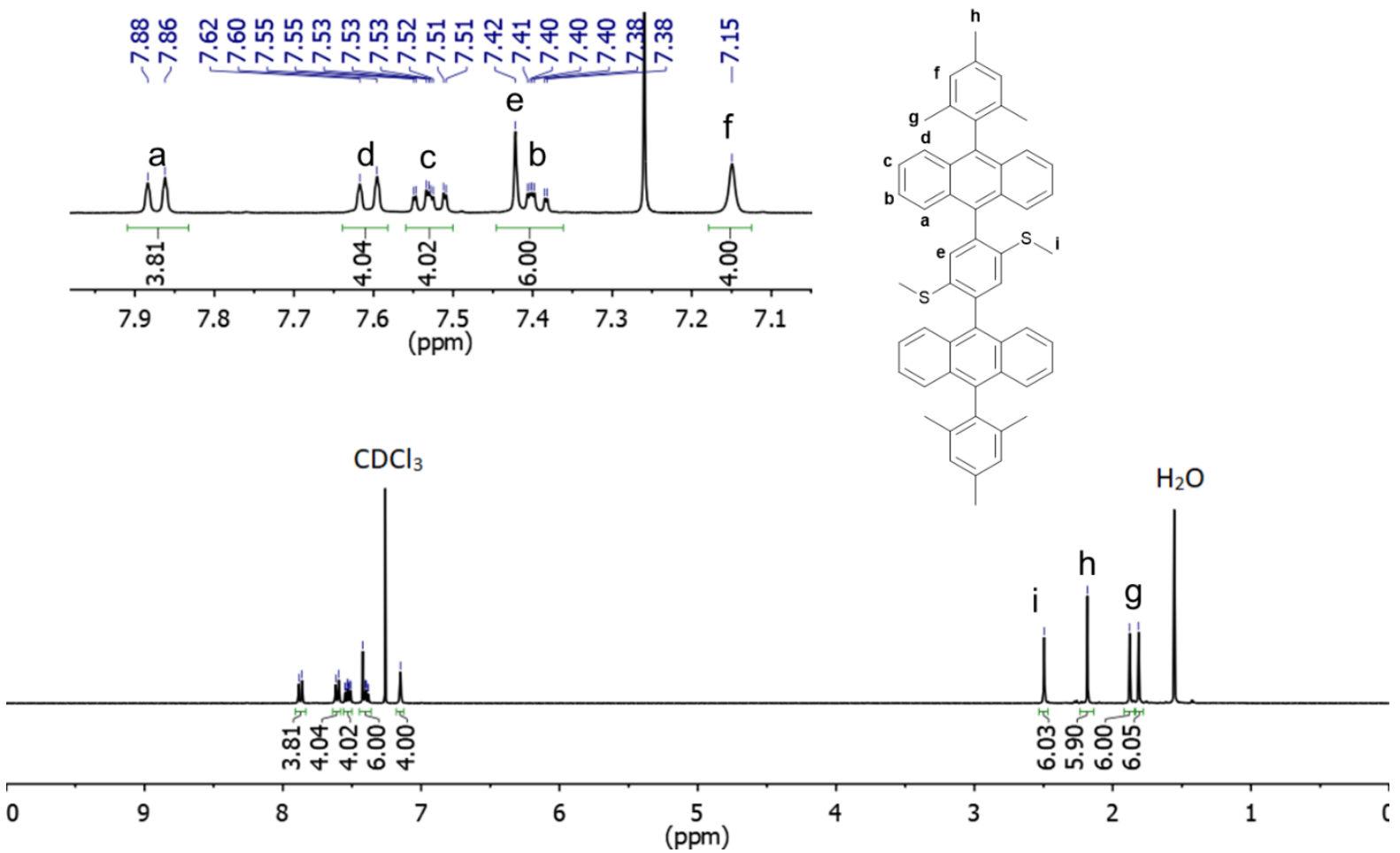

Figure S16. ${ }^{1} \mathrm{H}$ NMR spectrum of $7 \mathbf{a}\left(400 \mathrm{MHz}, \mathrm{CDCl}_{3}, \mathrm{rt}\right)$.

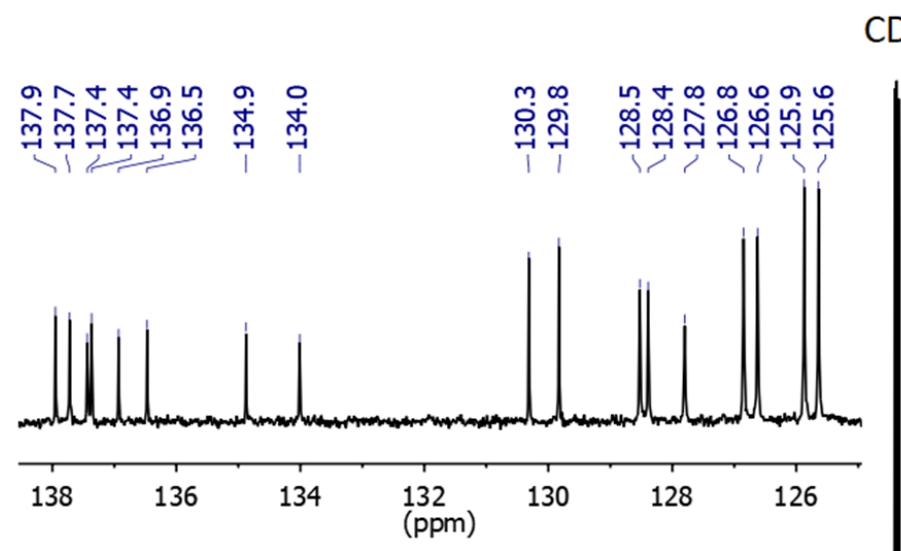

$\mathrm{CDCl}_{3}$

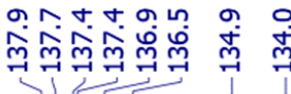

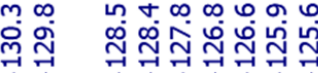
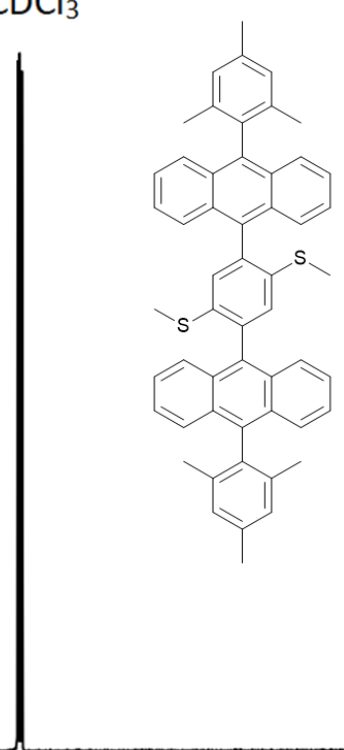

Hiin للف
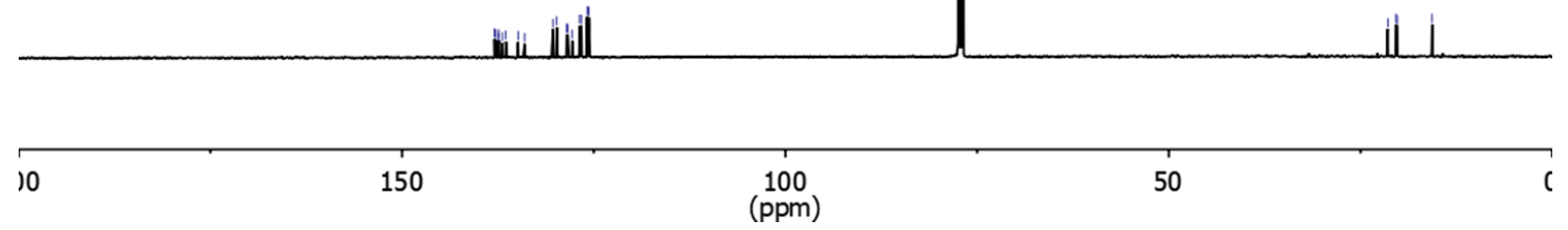

Figure S17. ${ }^{13} \mathrm{C}\left\{{ }^{1} \mathrm{H}\right\}$ NMR spectrum of $7 \mathbf{a}\left(500 \mathrm{MHz}, \mathrm{CDCl}_{3}\right.$, rt). 


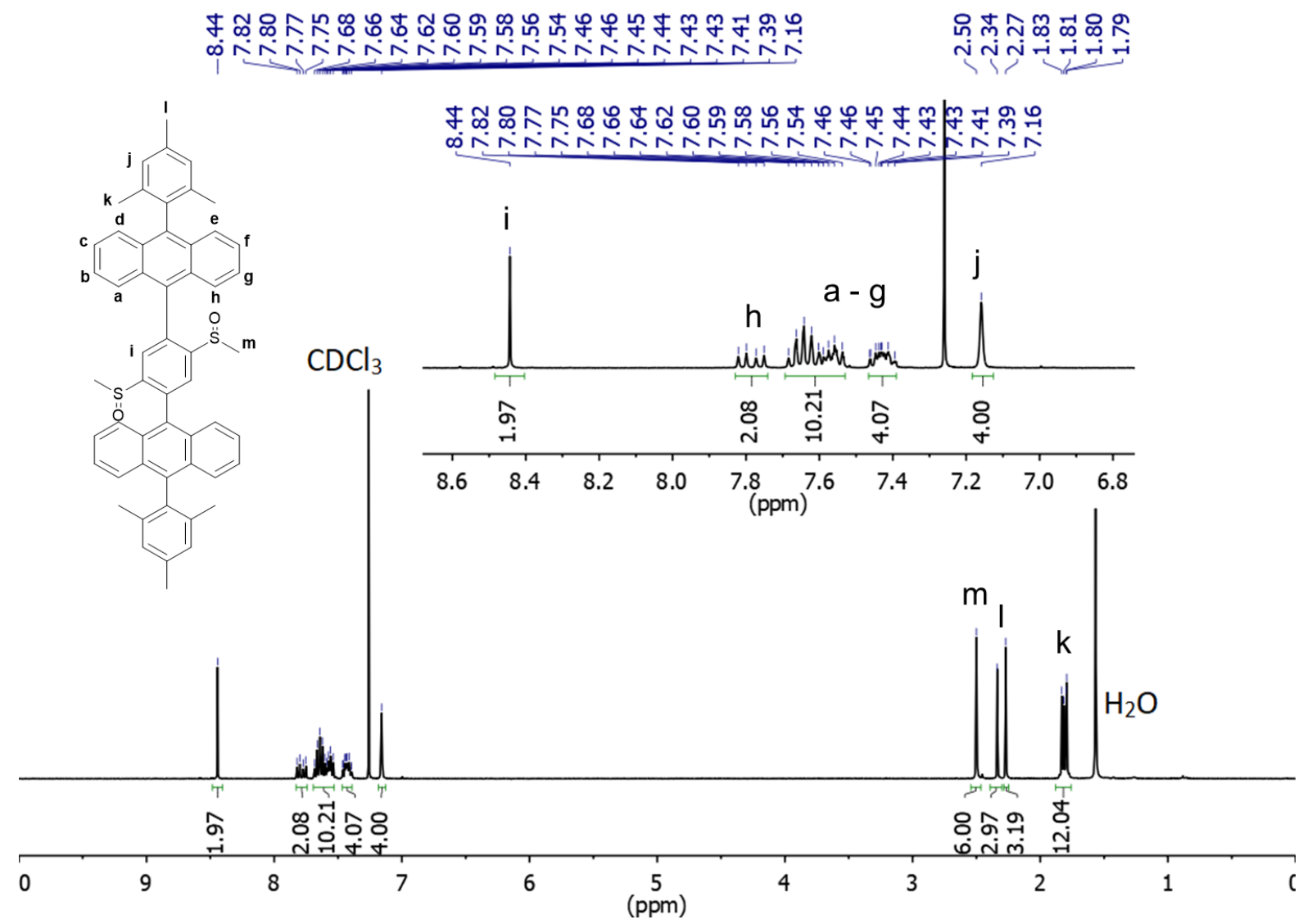

Figure S18. ${ }^{1} \mathrm{H}$ NMR spectrum of $8 \mathbf{a}\left(400 \mathrm{MHz}, \mathrm{CDCl}_{3}, \mathrm{rt}\right)$.

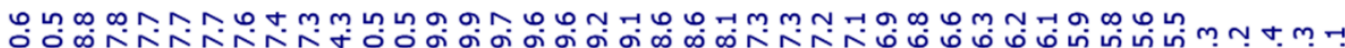

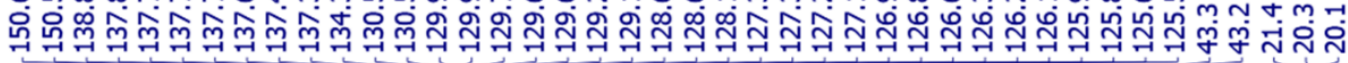

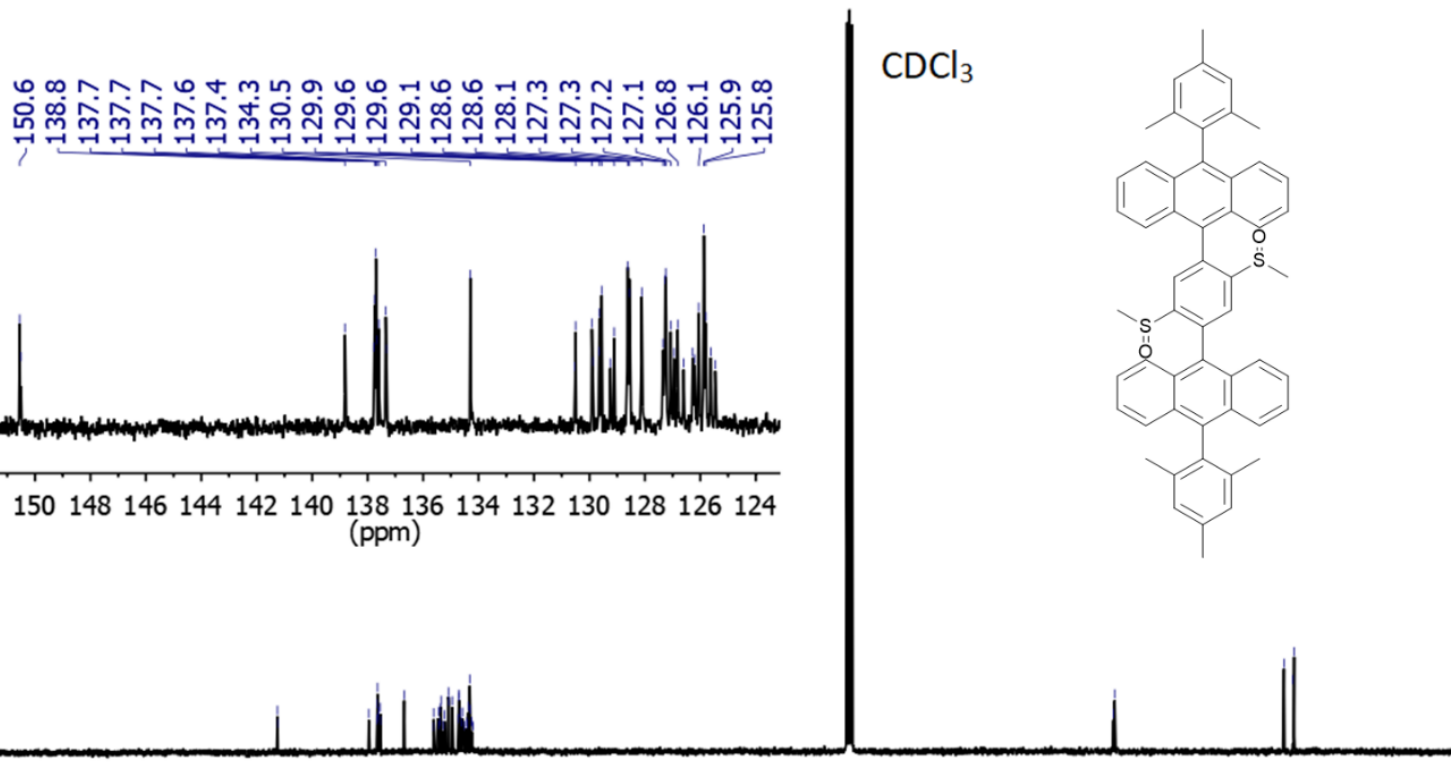

\begin{tabular}{|cccccccccc}
\hline 10 & 180 & 160 & 140 & 120 & $\begin{array}{c}100 \\
(\mathrm{ppm})\end{array}$ & 80 & 60 & 40 & 20
\end{tabular}

Figure S19. ${ }^{13} \mathrm{C}\left\{{ }^{1} \mathrm{H}\right\}$ NMR spectrum of $8 \mathbf{a}\left(400 \mathrm{MHz}, \mathrm{CDCl}_{3}, \mathrm{rt}\right)$. 


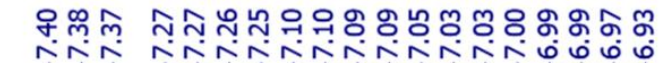
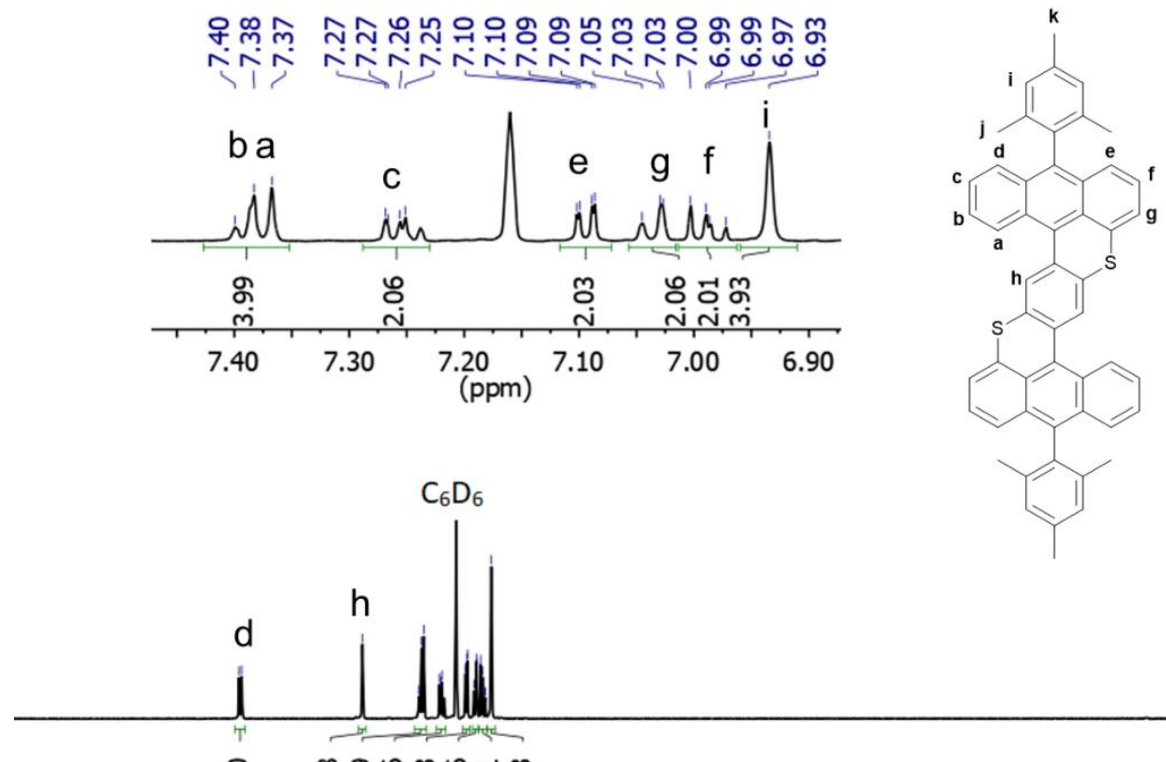

k

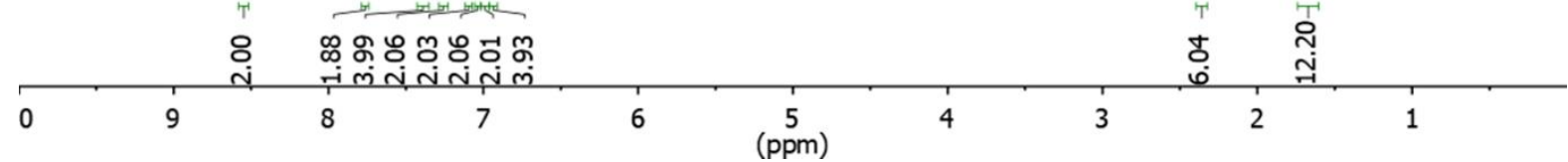

Figure S20. ${ }^{1} \mathrm{H}$ NMR spectrum of $9 \mathbf{a}\left(500 \mathrm{MHz}, \mathrm{CS}_{2} / \mathrm{C}_{6} \mathrm{D}_{6}, \mathrm{rt}\right)$.

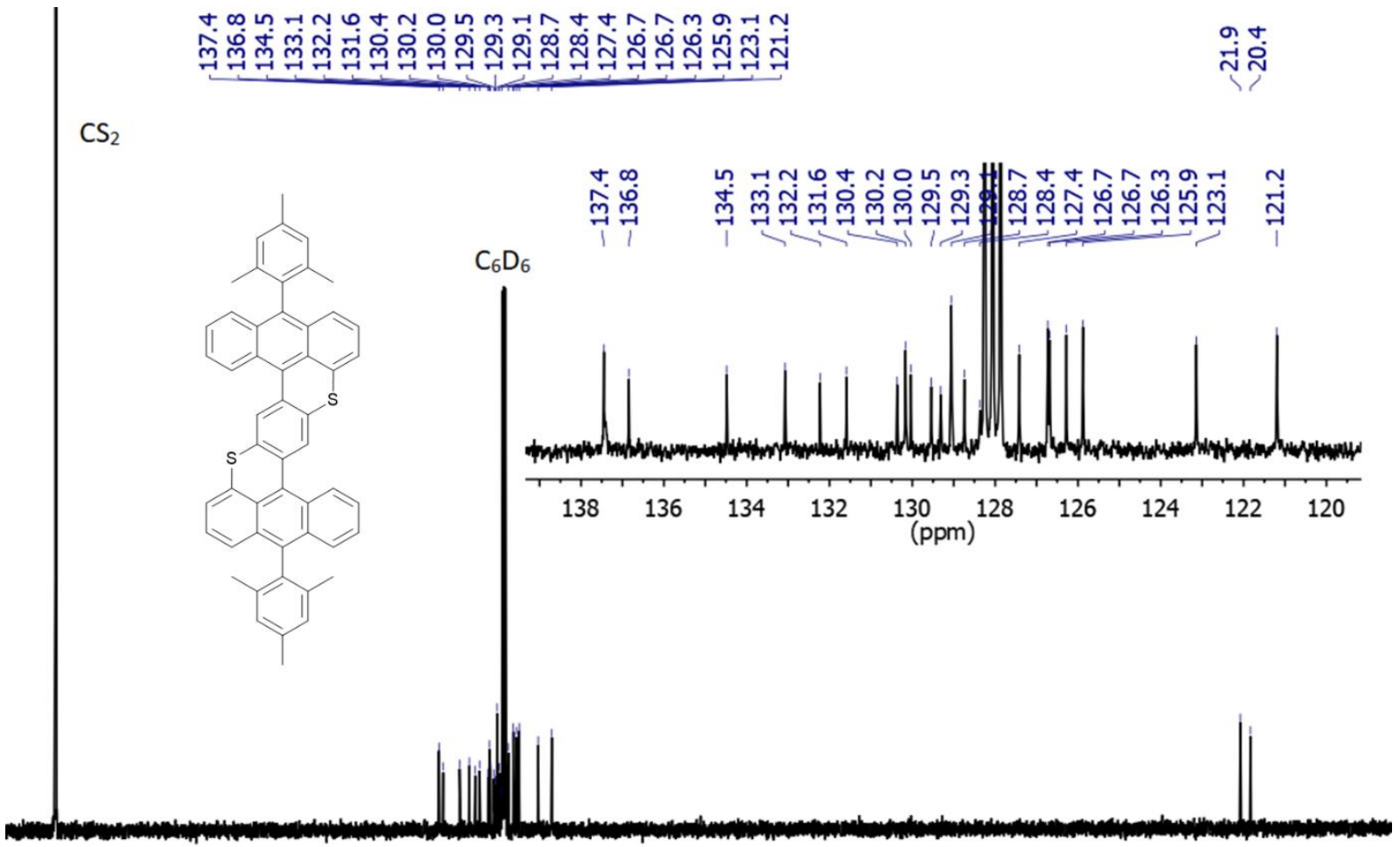

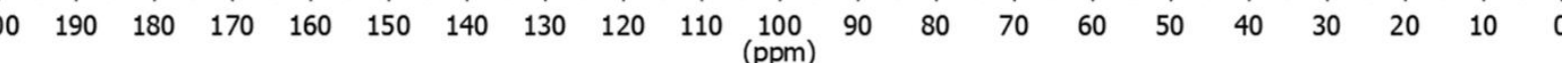

Figure S21. ${ }^{13} \mathrm{C}\left\{{ }^{1} \mathrm{H}\right\}$ NMR spectrum of 9a $\left(500 \mathrm{MHz}, \mathrm{CS}_{2} / \mathrm{C}_{6} \mathrm{D}_{6}, \mathrm{rt}\right)$. 

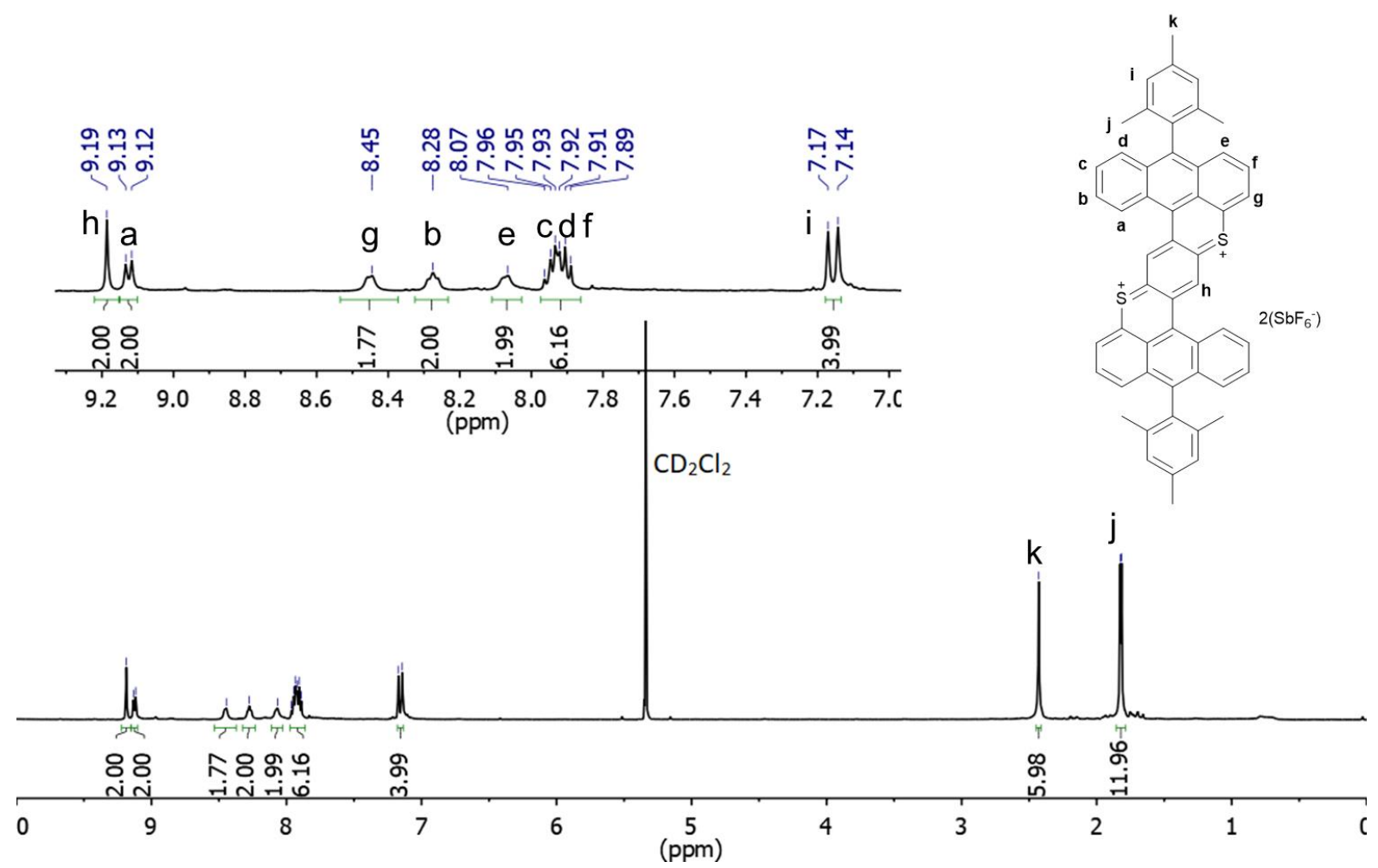

Figure S22. ${ }^{1} \mathrm{H}$ NMR spectrum of DBHZ-2S $\left(500 \mathrm{MHz}, \mathrm{CD}_{2} \mathrm{Cl}_{2},-80{ }^{\circ} \mathrm{C}\right)$.

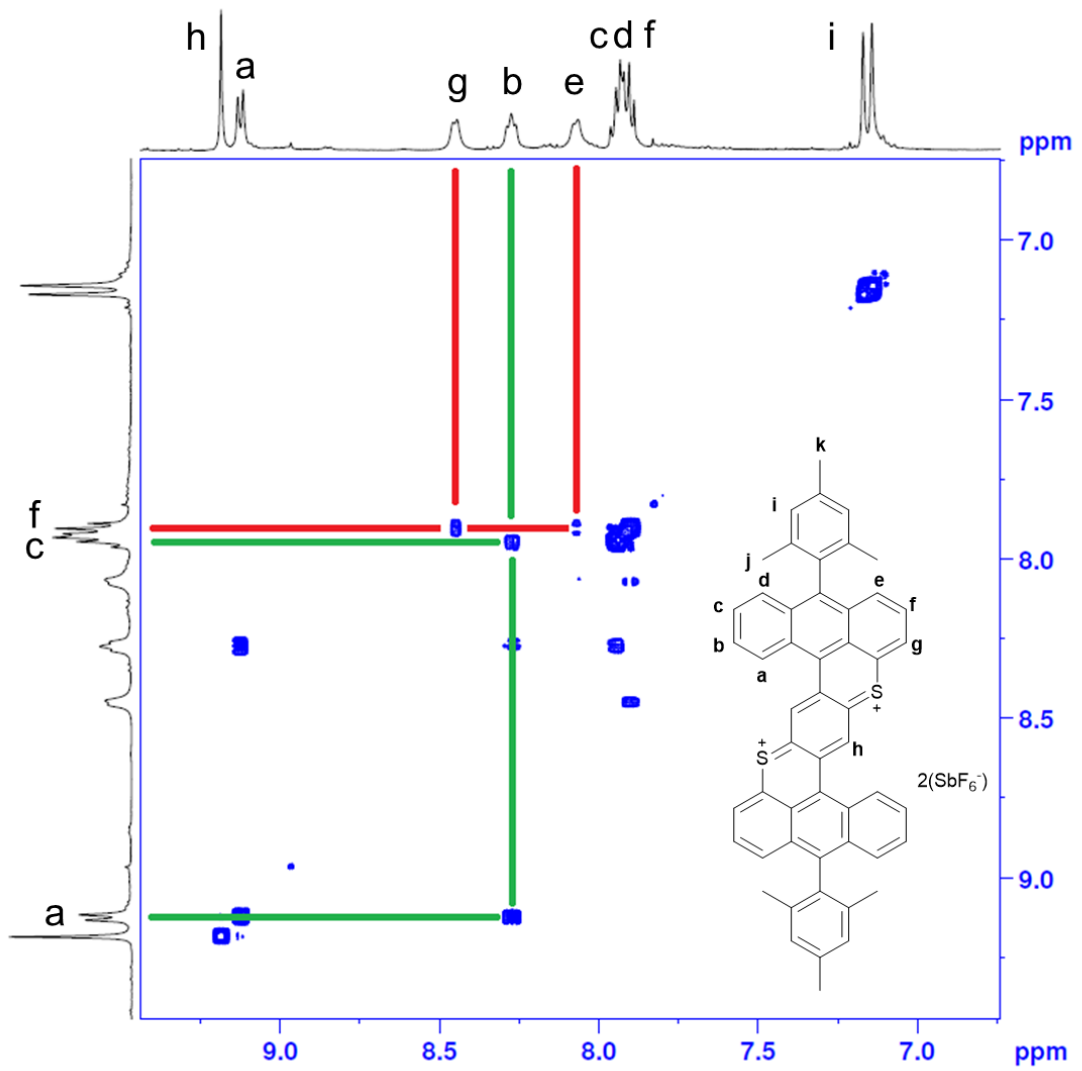

Figure S23. 2D COSY spectrum of DBHZ-2S $\left(500 \mathrm{MHz}, \mathrm{CD}_{2} \mathrm{Cl}_{2},-80{ }^{\circ} \mathrm{C}\right)$. 


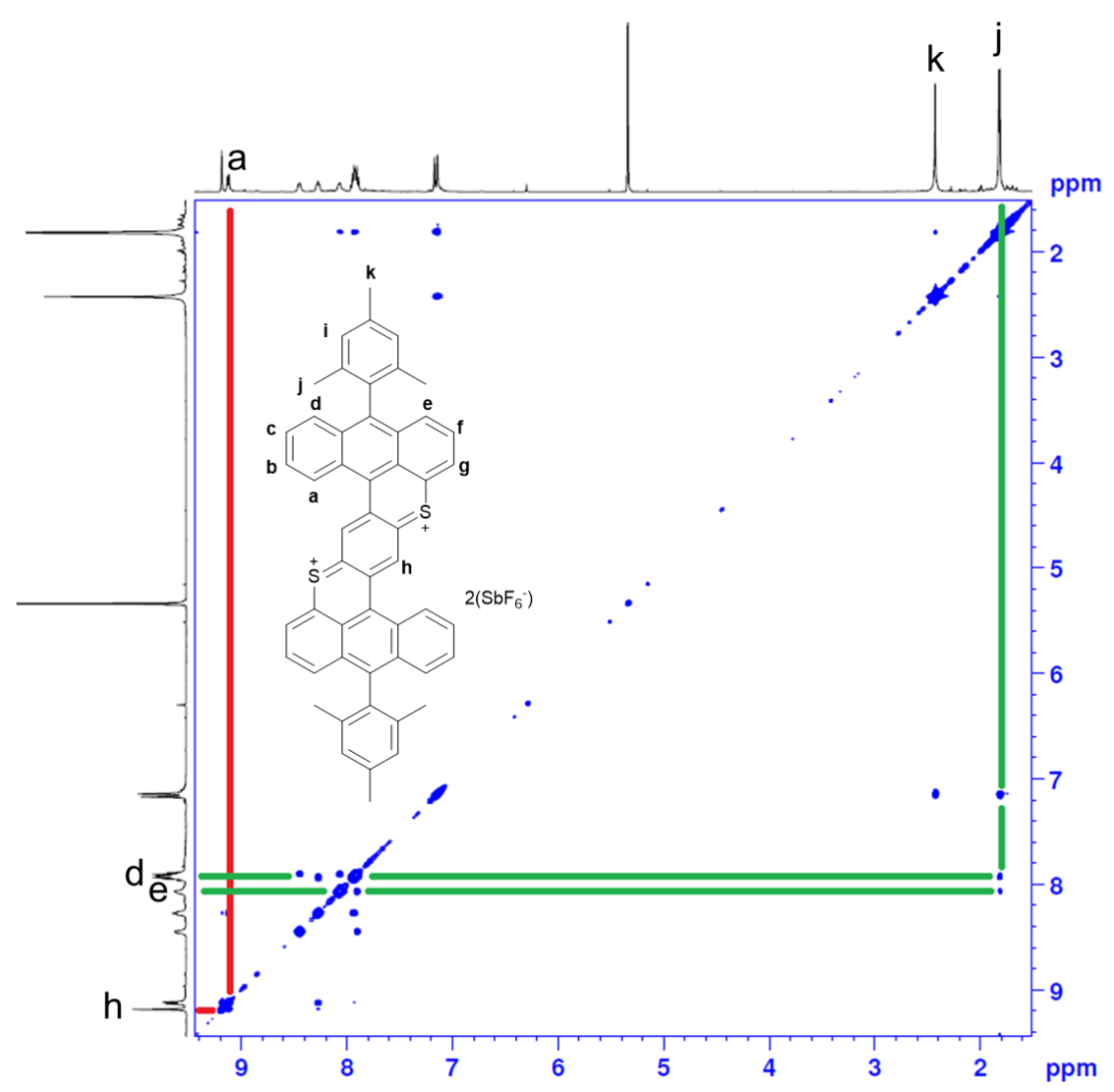

Figure S24. 2D NOESY spectrum of DBHZ-2S (500 $\left.\mathrm{MHz}, \mathrm{CD}_{2} \mathrm{Cl}_{2},-80{ }^{\circ} \mathrm{C}\right)$.

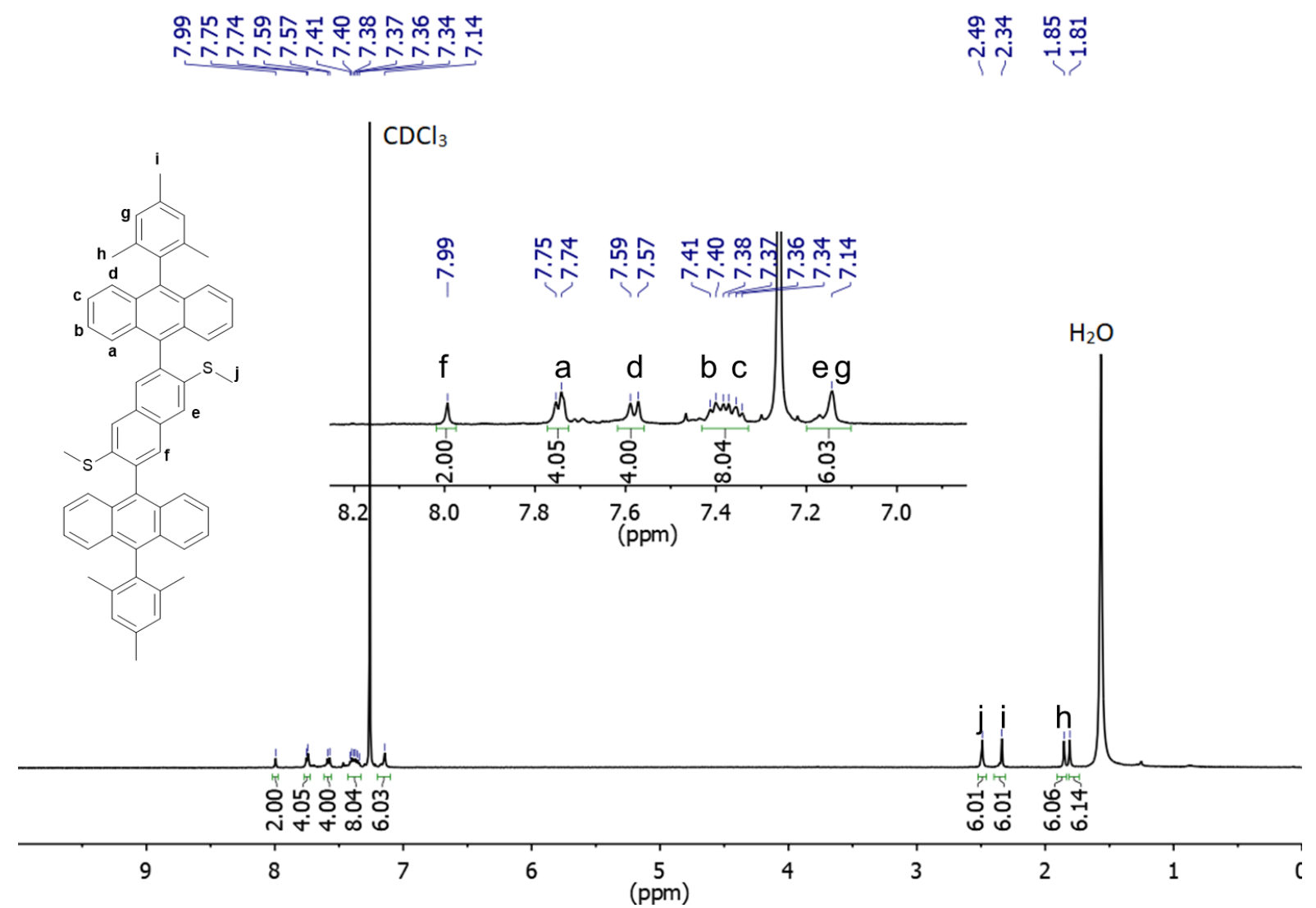

Figure S25. ${ }^{1} \mathrm{H}$ NMR spectrum of $\mathbf{7 b}\left(500 \mathrm{MHz}, \mathrm{CDCl}_{3}, \mathrm{rt}\right)$. 


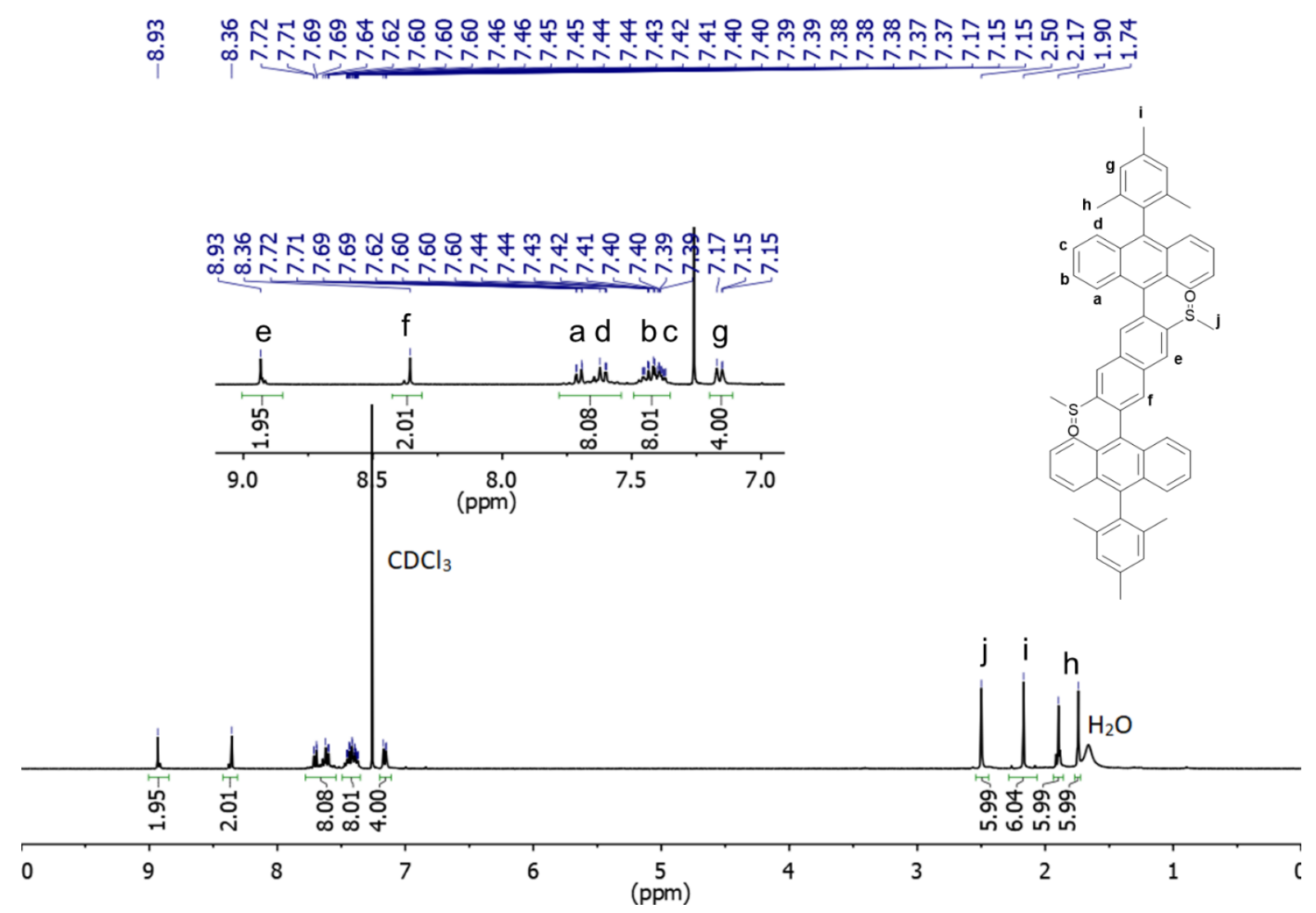

Figure S26. ${ }^{1} \mathrm{H}$ NMR spectrum of $\mathbf{8 b}\left(400 \mathrm{MHz}, \mathrm{CDCl}_{3}, \mathrm{rt}\right)$.

今. ñ

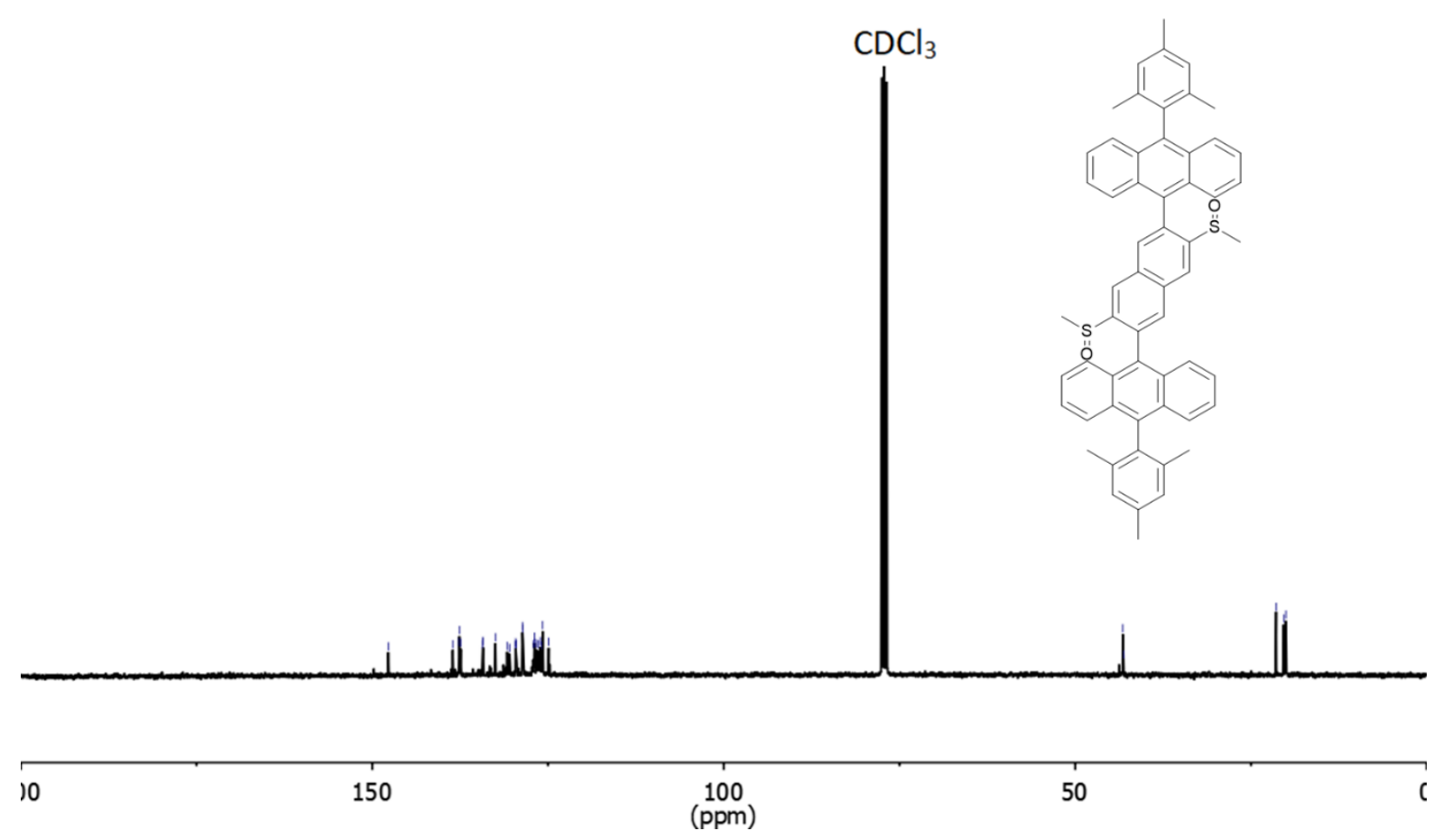

Figure S27. ${ }^{13} \mathrm{C}\left\{{ }^{1} \mathrm{H}\right\}$ NMR spectrum of $\mathbf{8 b}\left(400 \mathrm{MHz}, \mathrm{CDCl}_{3}, \mathrm{rt}\right)$. 

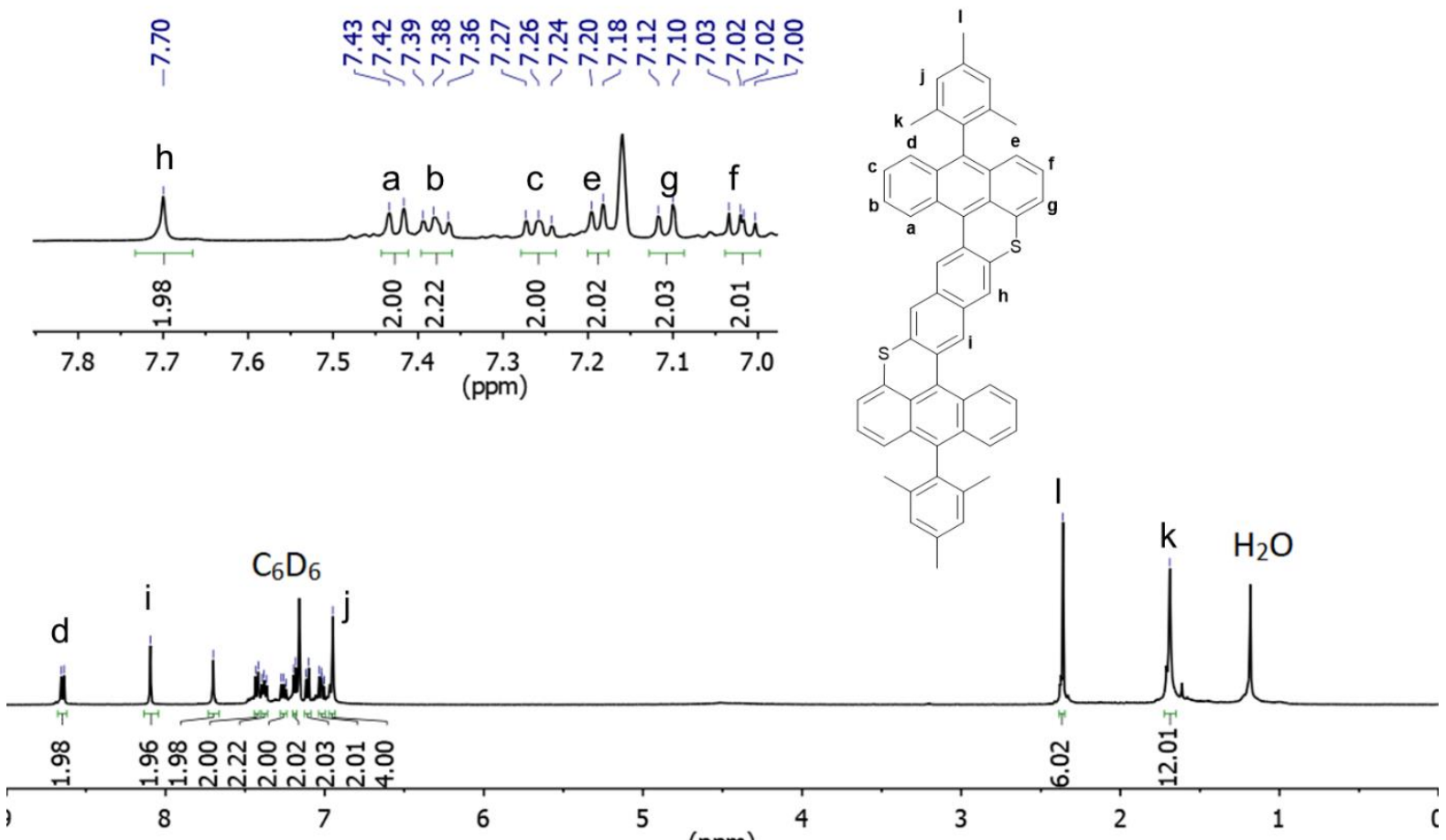

Figure S28. ${ }^{1} \mathrm{H}$ NMR spectrum of $9 \mathbf{b}\left(500 \mathrm{MHz}, \mathrm{CS}_{2} / \mathrm{C}_{6} \mathrm{D}_{6}, \mathrm{rt}\right)$.

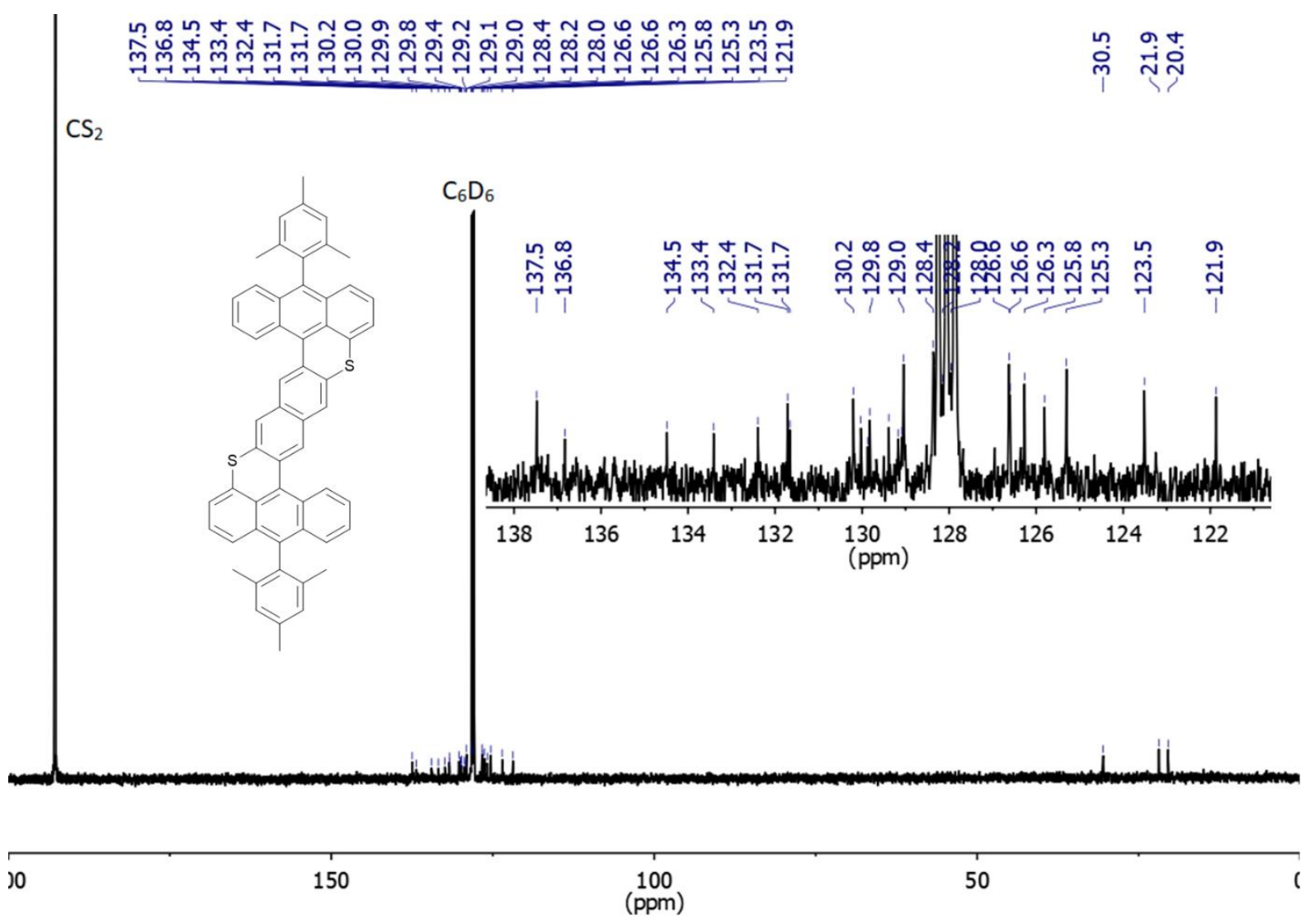

Figure S29. ${ }^{13} \mathrm{C}\left\{{ }^{1} \mathrm{H}\right\}$ NMR spectrum of $9 b\left(500 \mathrm{MHz}, \mathrm{CS}_{2} / \mathrm{C}_{6} \mathrm{D}_{6}, \mathrm{rt}\right)$. 


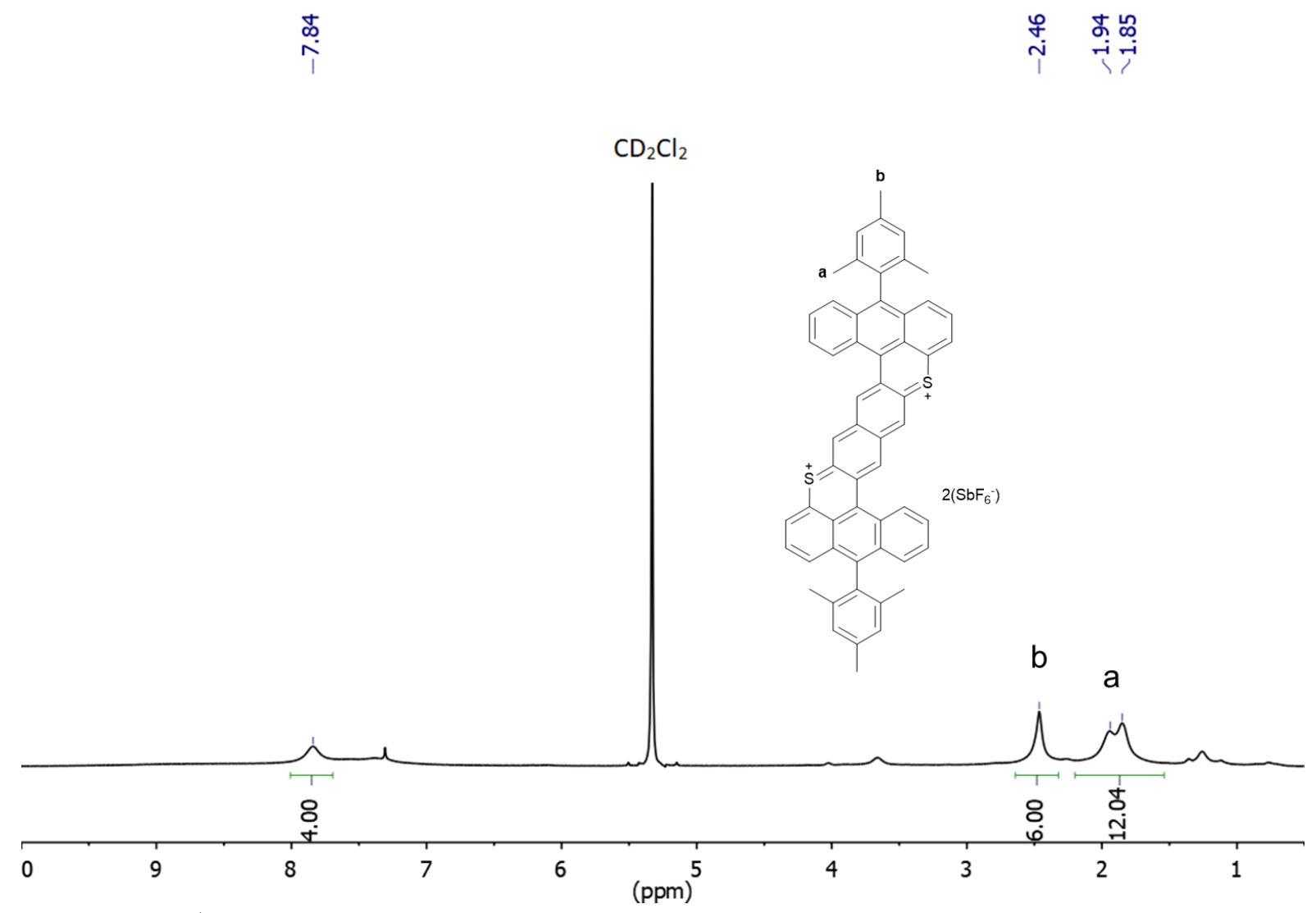

Figure S30. ${ }^{1} \mathrm{H}$ NMR spectrum of DBOZ-2S (500 MHz, $\left.\mathrm{CD}_{2} \mathrm{Cl}_{2},-80{ }^{\circ} \mathrm{C}\right)$.

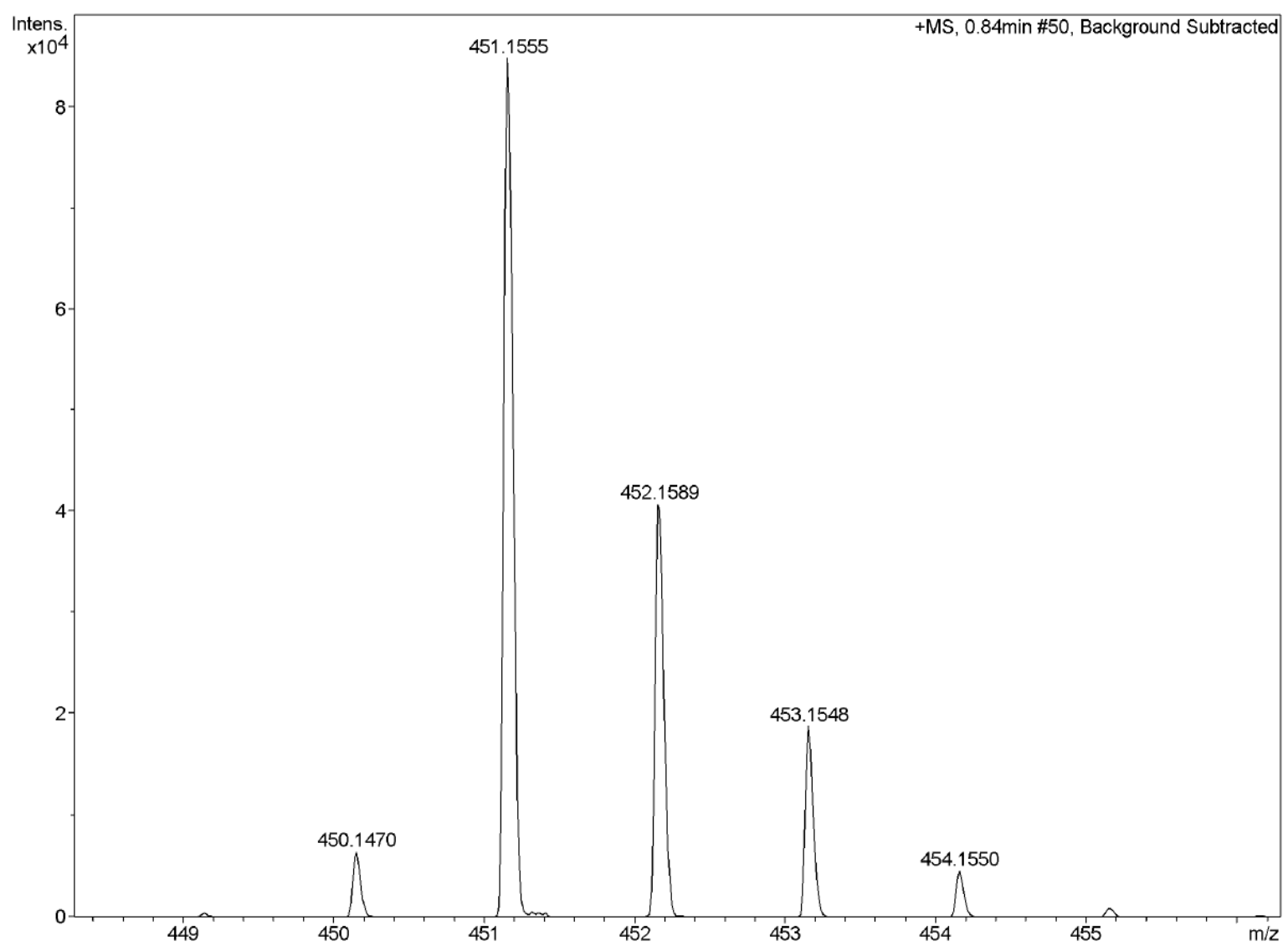

Figure S31. HR mass spectrum (APCI) of 3a. 


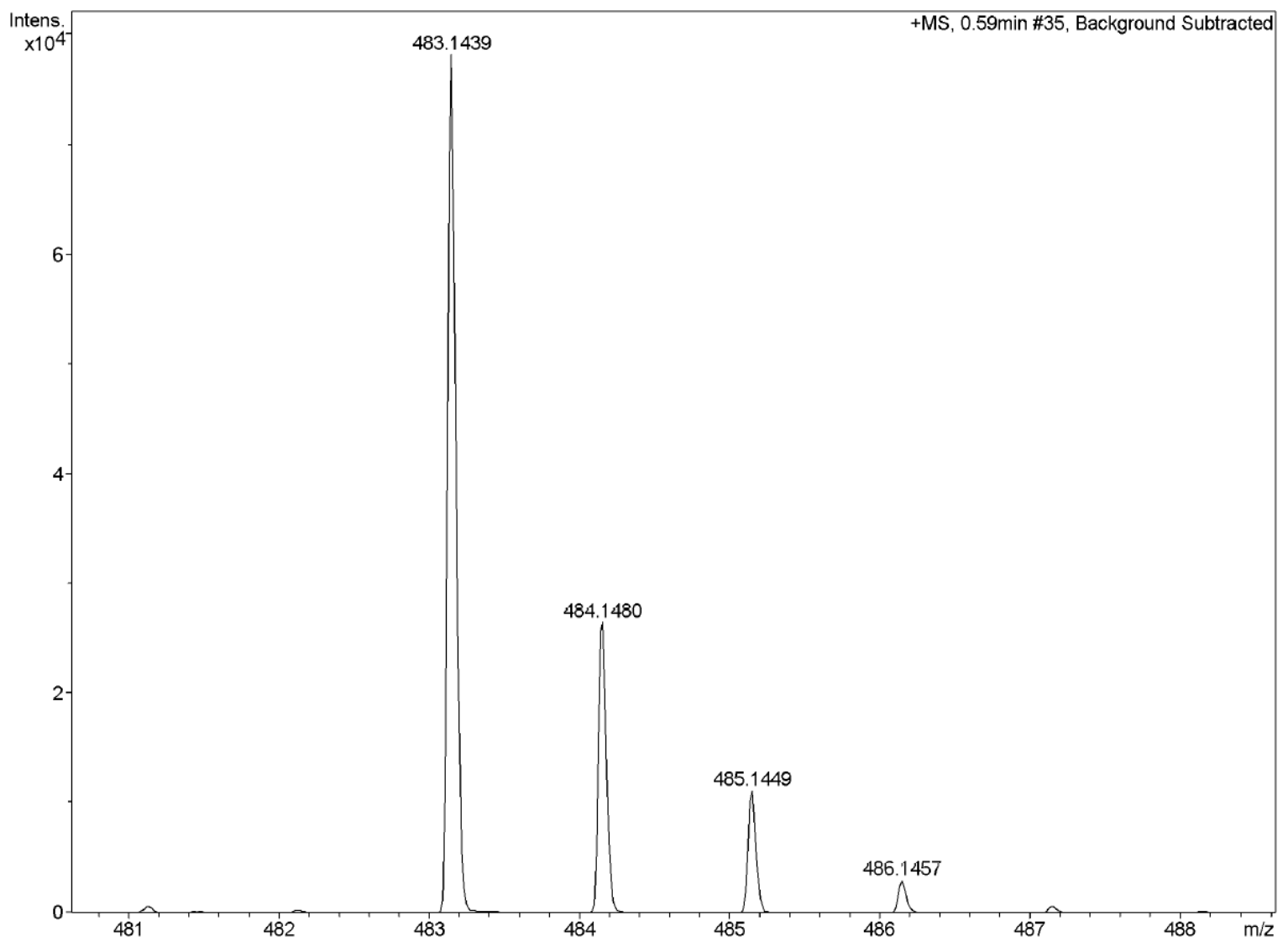

Figure S32. HR mass spectrum (APCI) of $\mathbf{4 a}$.

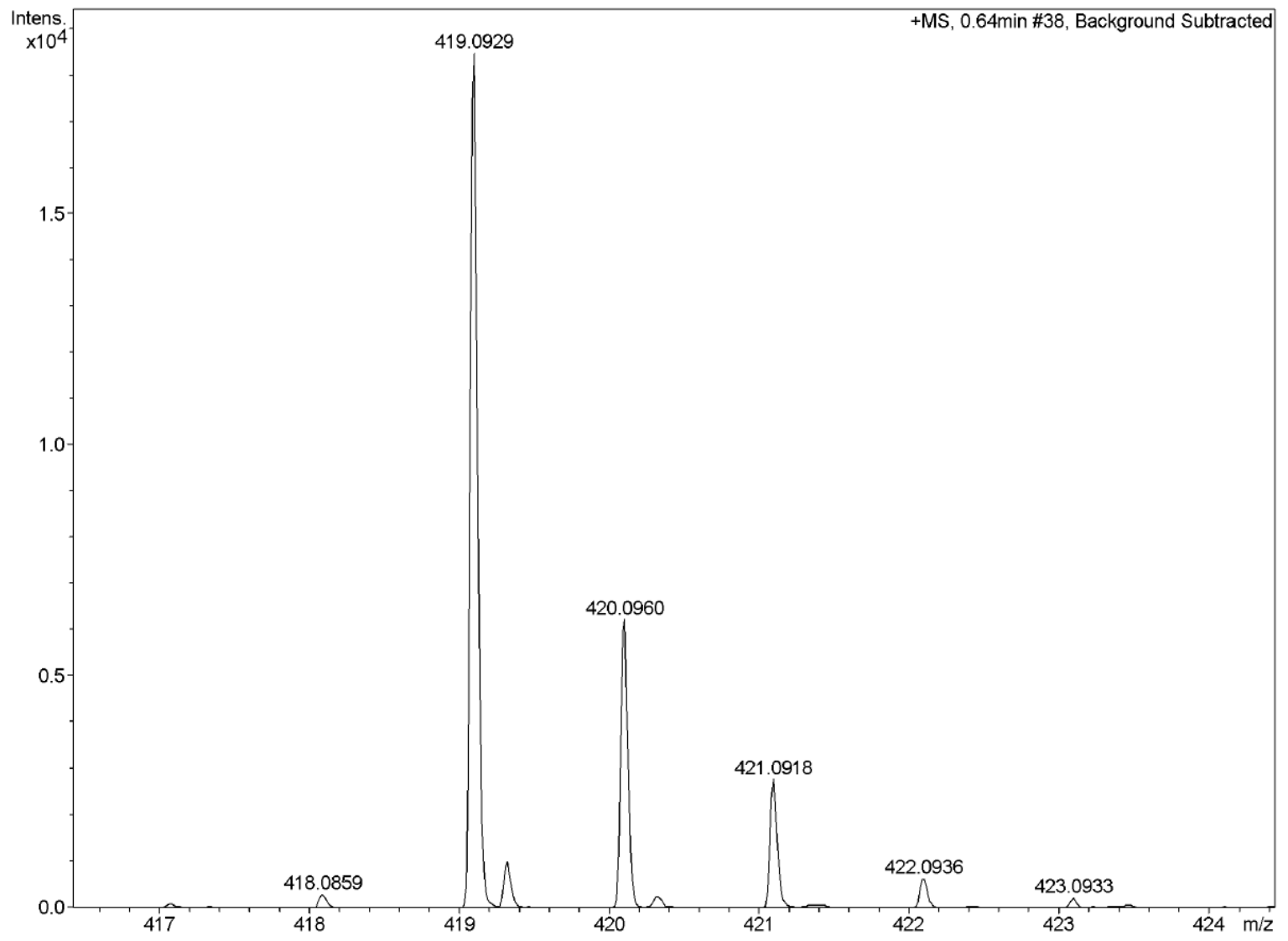

Figure S33. HR mass spectrum (APCI) of $\mathbf{5 a}$. 


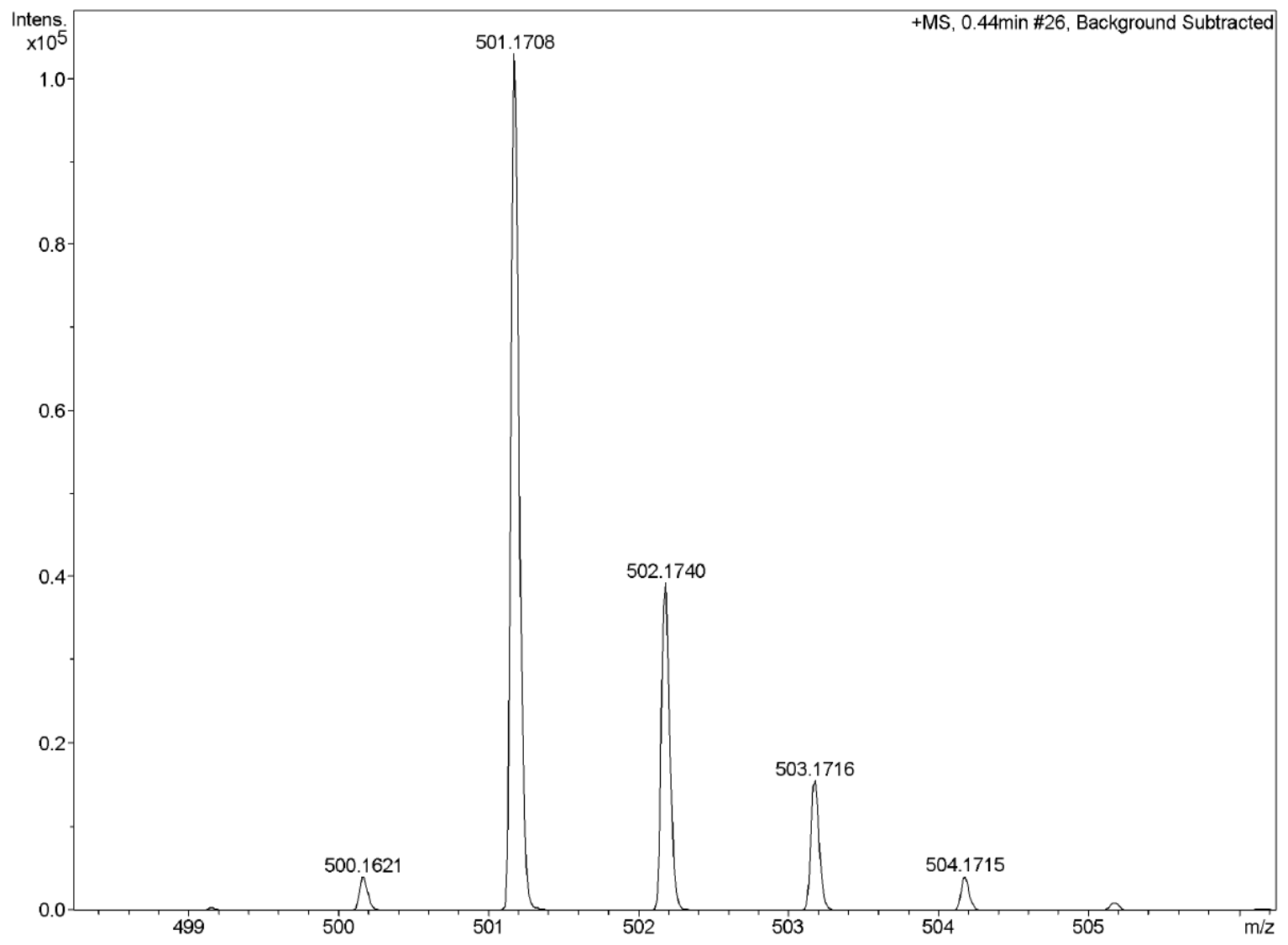

Figure S34. HR mass spectrum (APCI) of $\mathbf{3 b}$.

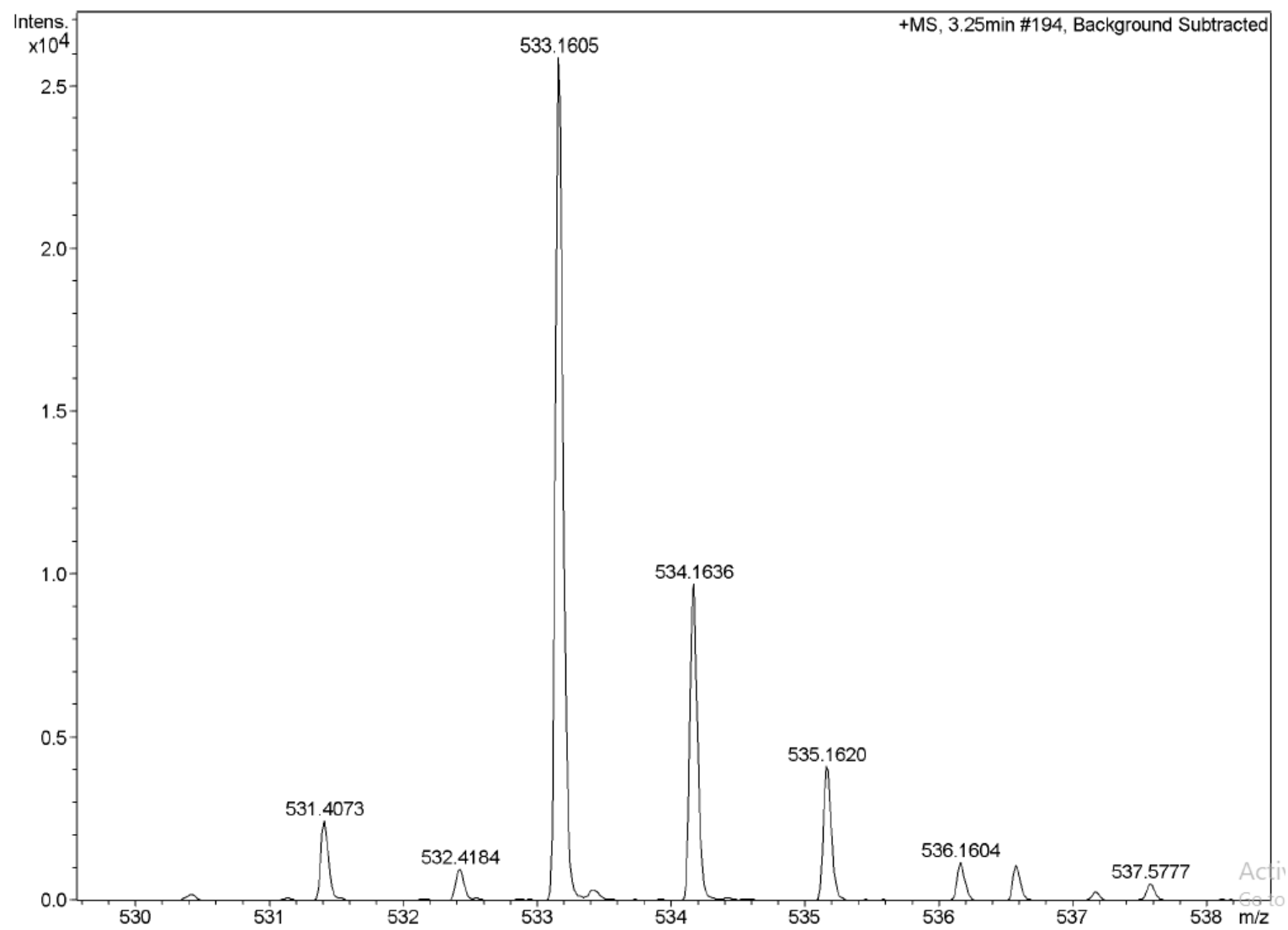

Figure S35. HR mass spectrum (APCI) of $\mathbf{4 b}$. 


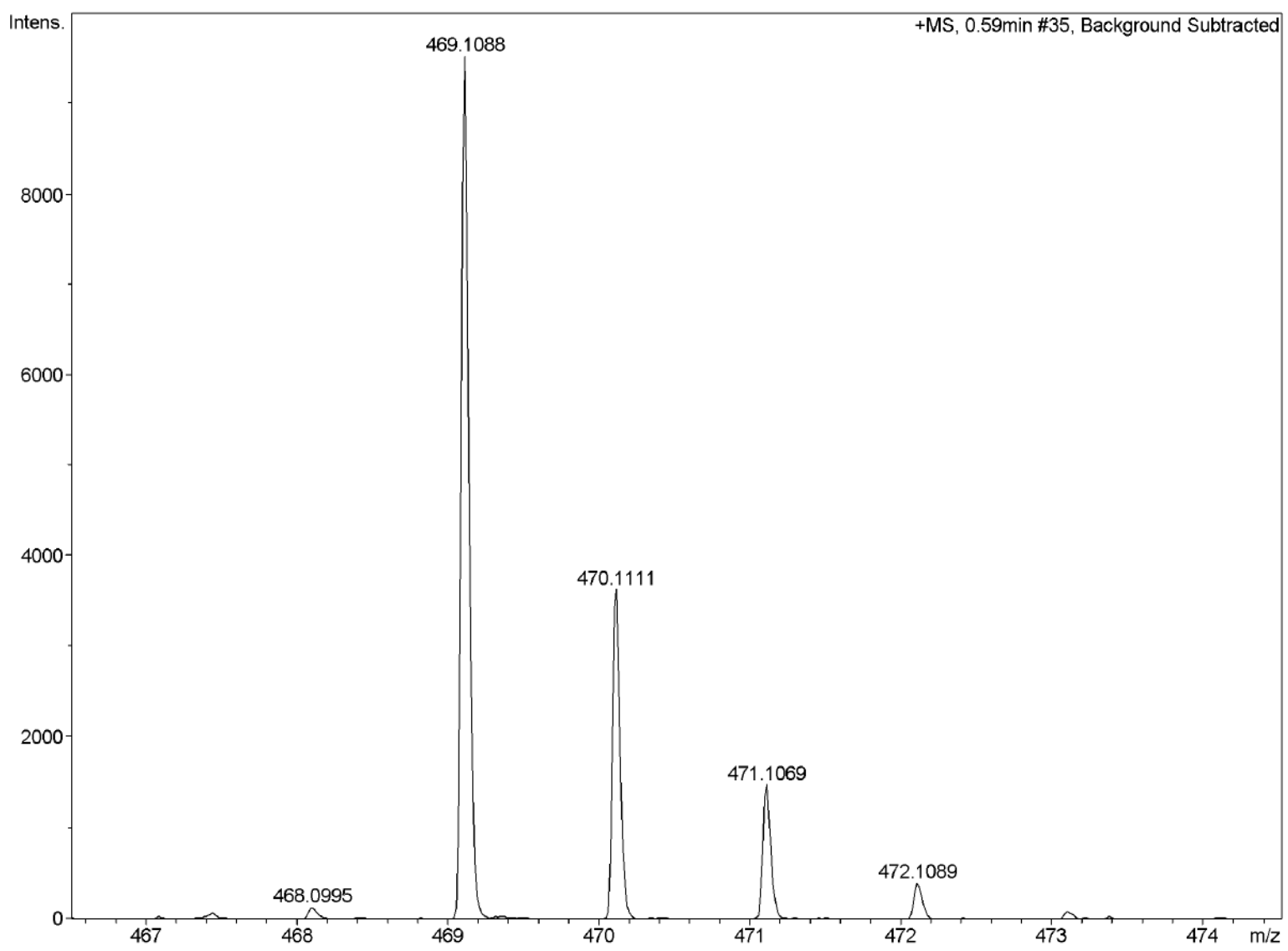

Figure S36. HR mass spectrum (APCI) of $\mathbf{5 b}$.

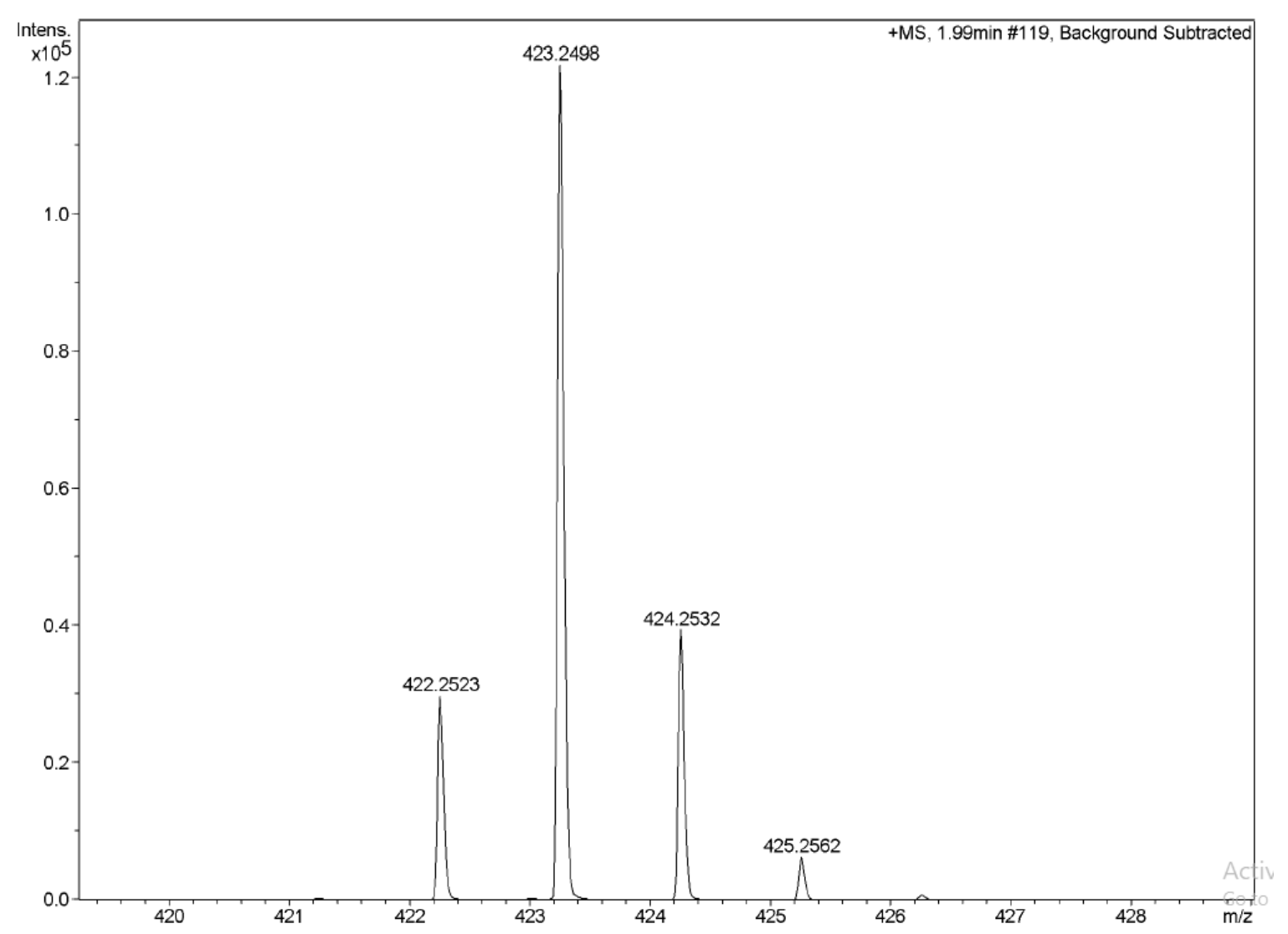

Figure S37. HR mass spectrum (APCI) of 6. 


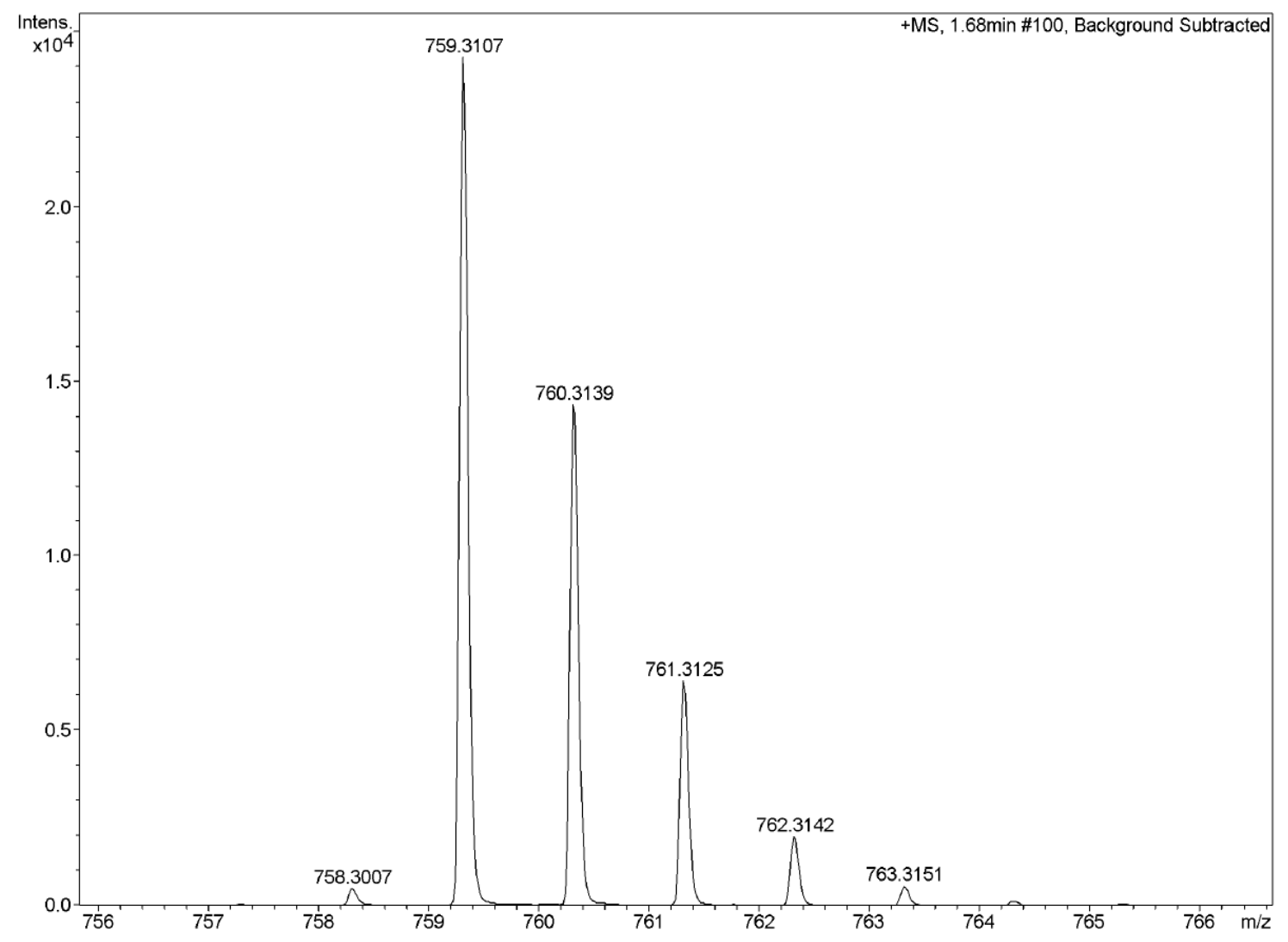

Figure S38. HR mass spectrum (APCI) of 7a.

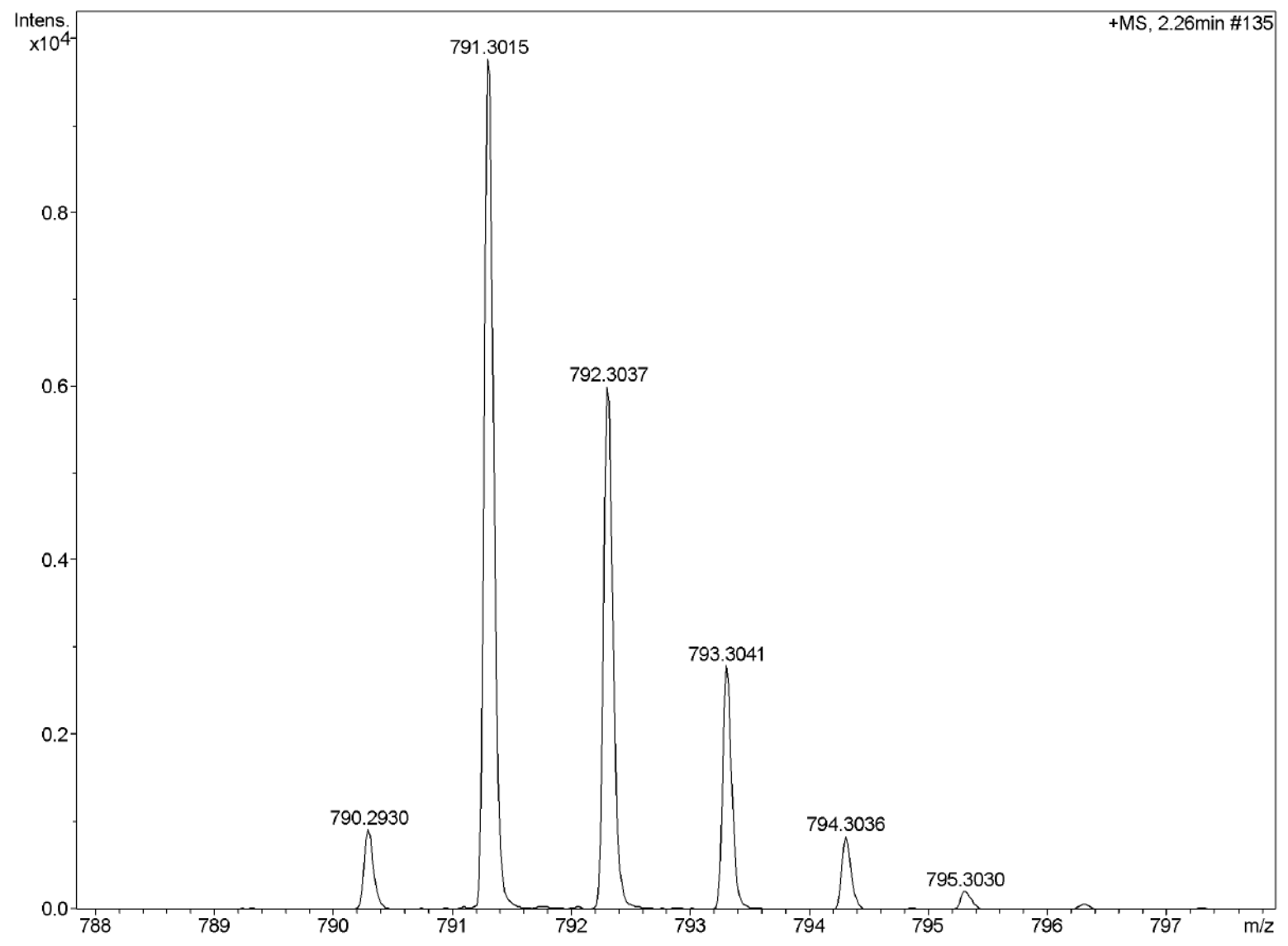

Figure S39. HR mass spectrum (APCI) of $8 \mathbf{a}$. 


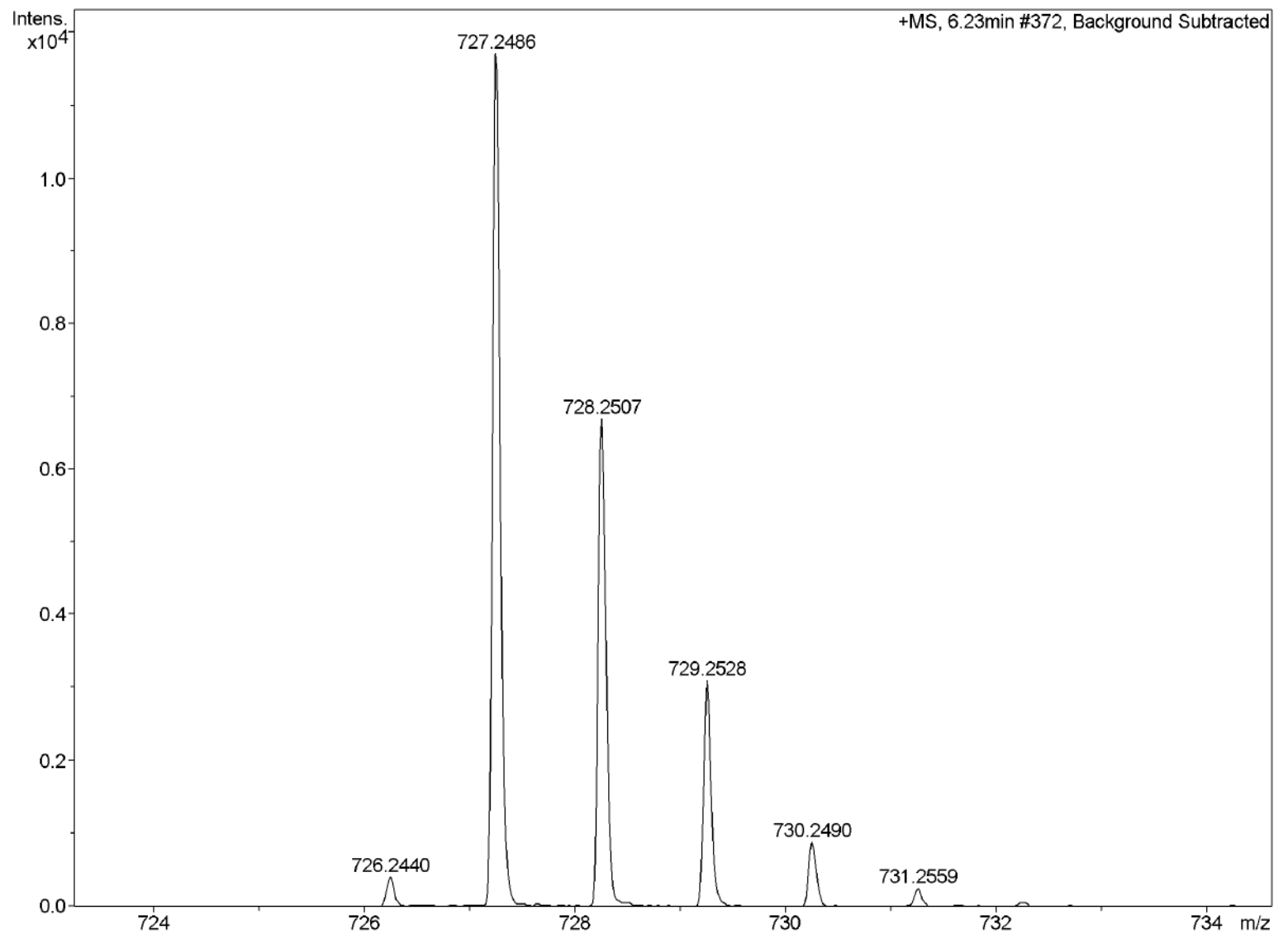

Figure S40. HR mass spectrum (APCI) of 9a.

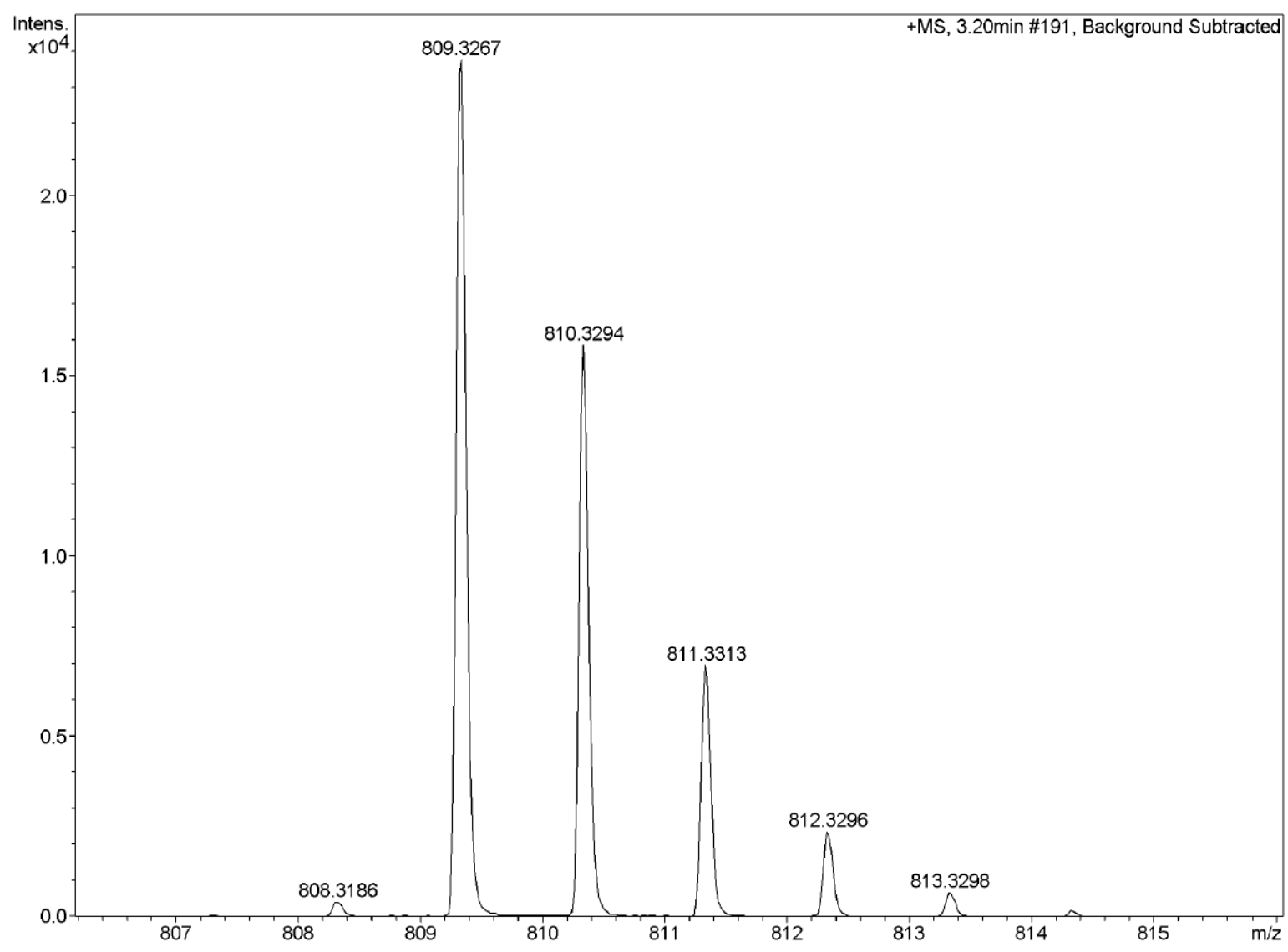

Figure S41. HR mass spectrum (APCI) of $7 \mathbf{b}$. 


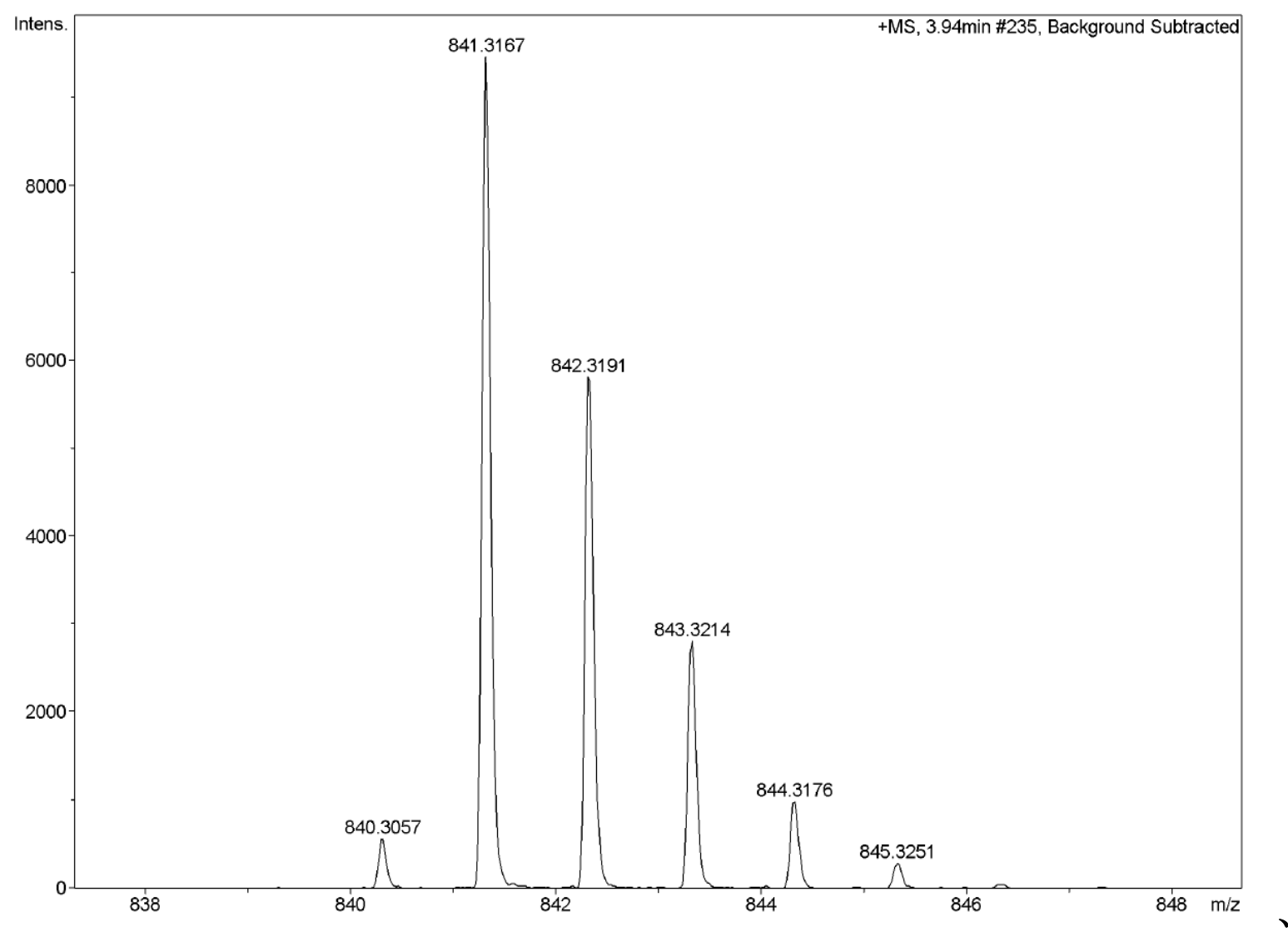

Figure S42. HR mass spectrum (APCI) of $\mathbf{8 b}$.

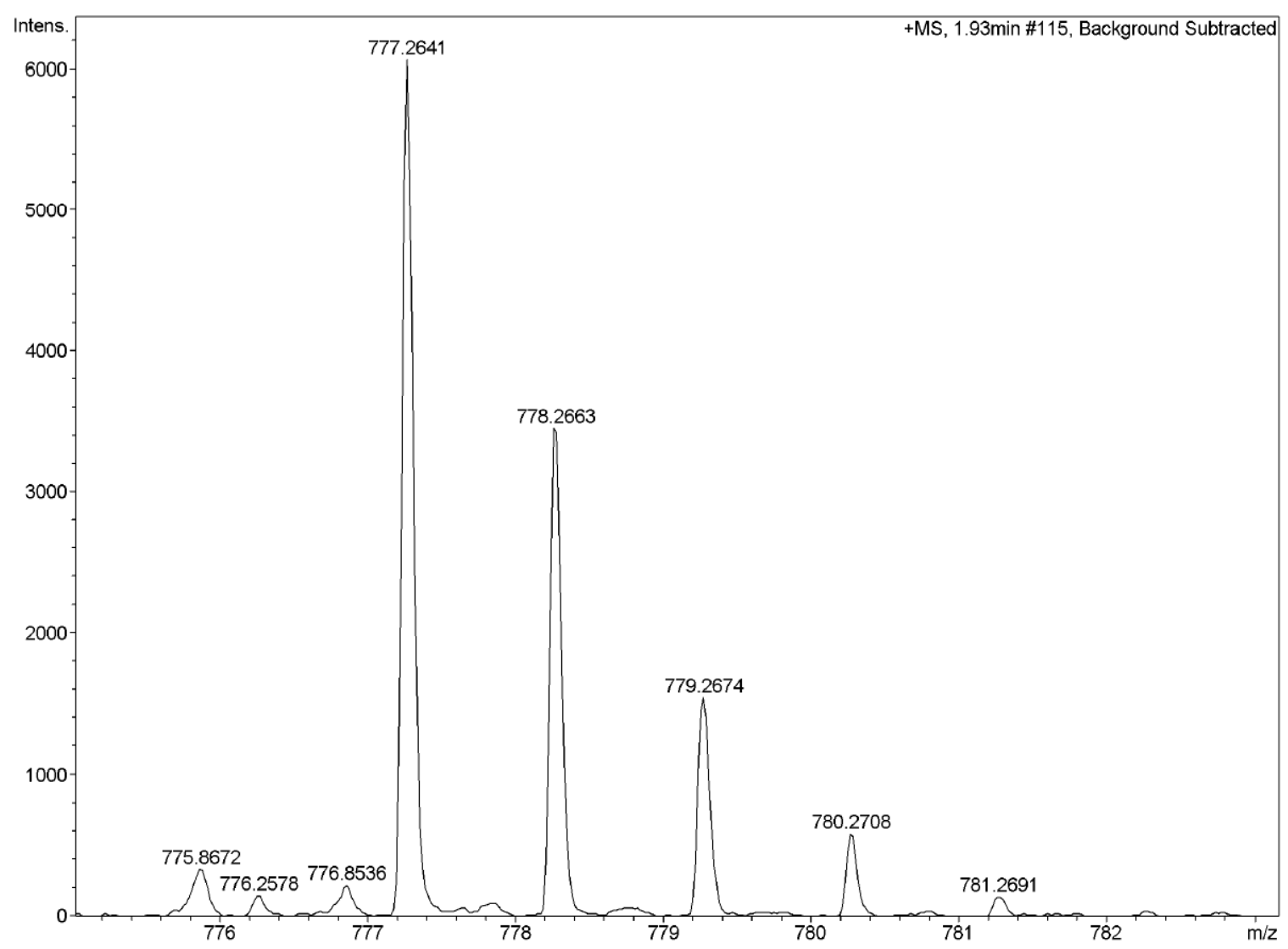

Figure S43. HR mass spectrum (APCI) of $\mathbf{9 b}$. 
4. X-ray crystallographic data of 5 a
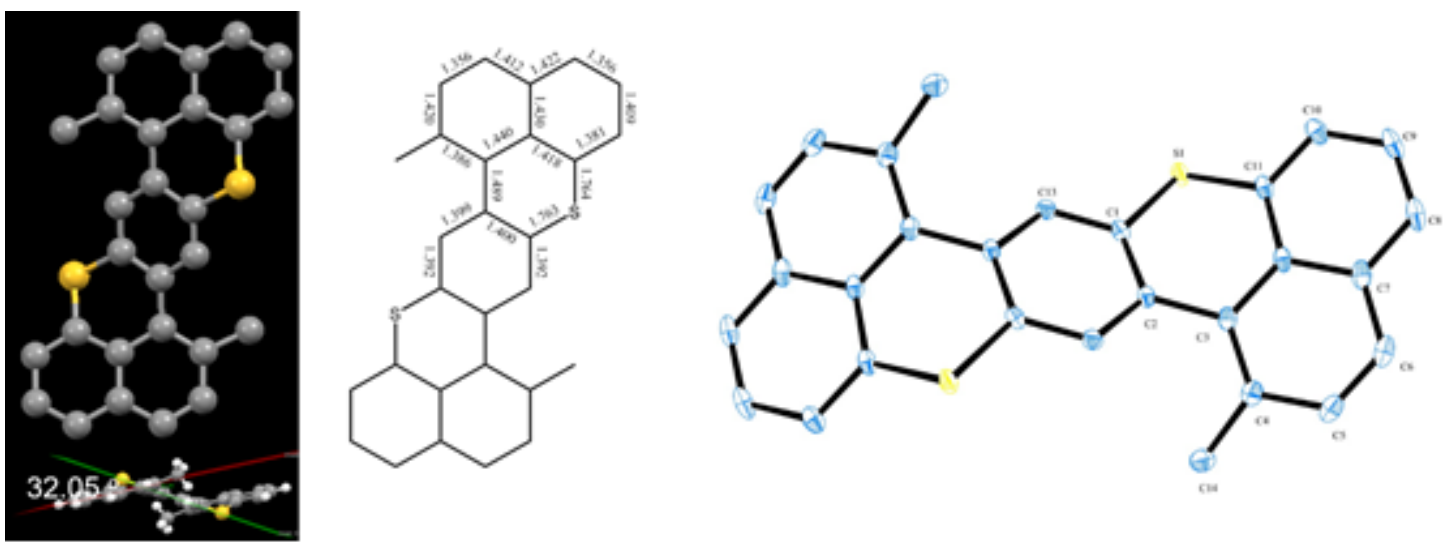

Figure S44. Crystal data and structure refinement for compound $\mathbf{5 a}$ with thermal ellipsoids at the $50 \%$ probability level. The single crystal of $\mathbf{5 a}$ was obtained by layer diffusion method with dichloromethane and hexane as binary solvent system.

Table S3. Sample and crystal data for 5a.

$\begin{array}{lll}\text { Identification code } & \mathbf{5 a} \\ \text { Chemical formula } & \mathrm{C}_{14} \mathrm{H} 9 \mathrm{~S} & 209.27 \mathrm{~g} / \mathrm{mol} \\ \text { Formula weight } & 100(2) \mathrm{K} \\ \text { Temperature } & 1.54178 \AA \\ \text { Wavelength } & (0.054 \times 0.056 \times 0.355) \mathrm{mm}^{3} \\ \text { Crystal size } & \text { monoclinic } & \\ \text { Crystal system } & \mathrm{P} 12(1) / \mathrm{c} 1 & \alpha=90^{\circ} \\ \text { Space group } & \mathrm{a}=8.148(6) \AA & \beta=94.014(19)^{\circ} \\ \text { Unit cell dimensions } & \mathrm{b}=8.347(4) \AA & \gamma=90^{\circ} \\ & \mathrm{c}=14.363(11) \AA & \\ \text { Volume } & 974.5(11) \AA^{3} & \\ \text { Z } & 4 & \\ \text { Density (calculated) } & 1.426 \mathrm{~g} / \mathrm{cm}^{3} & \\ \text { Absorption coefficient } & 2.559 \mathrm{~mm}^{-1} & \\ \text { F(000) } & 436 & \end{array}$


Table S4. Data collection and structure refinement for $\mathbf{5 a}$.

Theta range for data collection

Index ranges

Reflections collected

Independent reflections

Coverage of independent reflections

Absorption correction

Max. and min.

transmission

Refinement method

Refinement program

Function minimized

Data / restraints / parameters

Goodness-of-fit on $\mathbf{F}^{2}$

$\Delta / \sigma_{\max }$

Final $\mathbf{R}$ indices

Weighting scheme

Largest diff. peak and hole

R.M.S. deviation from mean
5.44 to $74.50^{\circ}$

$-10<=\mathrm{h}<=10,-10<=\mathrm{k}<=9,-17<=\mathrm{l}<=17$

8859

$1954[\mathrm{R}(\mathrm{int})=0.0393]$

$98.1 \%$

Multi-Scan

0.8740 and 0.4640

Full-matrix least-squares on $\mathrm{F}^{2}$

SHELXL-2014/7 (Sheldrick, 2014)

$\Sigma \mathrm{w}\left(\mathrm{F}_{\mathrm{o}}^{2}-\mathrm{F}_{\mathrm{c}}^{2}\right)^{2}$

1954 / 0 / 137

1.063

0.001

1684 data; $\mathrm{I}>2 \sigma(\mathrm{I}) \begin{aligned} & \mathrm{R} 1=0.0373, \mathrm{wR} 2= \\ & 0.0793\end{aligned}$

all data $\mathrm{R} 1=0.0471, \mathrm{wR} 2=$ 0.0835

$\mathrm{w}=1 /\left[\sigma^{2}\left(\mathrm{~F}_{\mathrm{o}}^{2}\right)+(0.0279 \mathrm{P})^{2}+0.8263 \mathrm{P}\right]$ where $\mathrm{P}=\left(\mathrm{F}_{\mathrm{o}}{ }^{2}+2 \mathrm{~F}_{\mathrm{c}}{ }^{2}\right) / 3$

0.305 and $-0.280 \mathrm{e}^{-3}$

$0.061 \mathrm{e}^{-3}$ 
Table S5. Atomic coordinates and equivalent isotropic atomic displacement parameters $\left(\AA^{2}\right)$ for $\mathbf{5 a}$.

$U(e q)$ is defined as one third of the trace of the orthogonalized $U_{i j}$ tensor.

$\mathbf{x} / \mathbf{a}$

$0.17860(5)$

$0.3576(2)$

$0.4152(2)$

$0.3350(2)$

$0.3503(2)$

$0.3093(2)$

$0.2521(2)$

$0.2144(2)$

$0.1360(2)$

$0.0895(2)$

$0.1137(2)$

0.1890(2)

$0.2495(2)$

$0.4384(2)$

$0.4008(2)$ $\mathbf{y} / \mathbf{b}$

$\mathbf{z} / \mathbf{c}$

$0.40879(3)$

$0.45861(11)$

$0.42146(11)$

$0.33619(11)$

0.31591(12)

$0.22338(13)$

$0.15438(13)$

$0.17500(12)$

$0.10759(12)$

0.13103(12)

$0.22389(12)$

0.29109(11)

$0.26829(11)$

0.53597(11)

$0.38725(13)$
$\mathbf{U}(\mathbf{e q})$

0.02021(14)

$0.0172(4)$

0.0161(4)

$0.0169(4)$

0.0204(4)

$0.0239(4)$

$0.0248(4)$

$0.0216(4)$

$0.0254(5)$

$0.0266(5)$

$0.0226(4)$

$0.0188(4)$

$0.0178(4)$

$0.0168(4)$

$0.0263(4)$

Table S6. Bond lengths ( $\mathrm{A})$ for $5 \mathbf{a}$.

$\begin{array}{llll}\text { S1-C1 } & 1.763(2) & \text { S1-C11 } & 1.764(2) \\ \text { C1-C13 } & 1.392(2) & \text { C1-C2 } & 1.400(3) \\ \text { C2-C13 } & 1.399(2) & \text { C2-C3 } & 1.489(2) \\ \text { C3-C4 } & 1.386(3) & \mathrm{C} 3-\mathrm{C} 12 & 1.440(2) \\ \text { C4-C5 } & 1.420(3) & \text { C4-C14 } & 1.507(3)\end{array}$




$\begin{array}{llll}\text { C5-C6 } & 1.356(3) & \mathrm{C} 5-\mathrm{H} 5 & 0.95 \\ \text { C6-C7 } & 1.412(3) & \mathrm{C} 6-\mathrm{H} 6 & 0.95 \\ \text { C7-C8 } & 1.422(3) & \mathrm{C} 7-\mathrm{C} 12 & 1.430(3) \\ \text { C8-C9 } & 1.356(3) & \mathrm{C} 8-\mathrm{H} 8 & 0.95 \\ \text { C9-C10 } & 1.409(3) & \mathrm{C} 9-\mathrm{H} 9 & 0.95 \\ \text { C10-C11 } & 1.381(3) & \mathrm{C} 10-\mathrm{H} 10 & 0.95 \\ \text { C11-C12 } & 1.418(3) & \mathrm{C} 13-\mathrm{C} 2 & 1.399(2) \\ \text { C13-H13 } & 0.95 & \mathrm{C} 14-\mathrm{H} 14 \mathrm{~A} & 0.98 \\ \text { C14-H14B } & 0.98 & \mathrm{C} 14-\mathrm{H} 14 \mathrm{C} & 0.98\end{array}$

Table S7. Bond angles $\left(^{\circ}\right.$ ) for $\mathbf{5 a}$.

\begin{tabular}{llll}
\hline C1-S1-C11 & $100.10(9)$ & C13-C1-C2 & $121.70(16)$ \\
C13-C1-S1 & $117.40(15)$ & C2-C1-S1 & $120.90(13)$ \\
C13-C2-C1 & $116.28(16)$ & C13-C2-C3 & $121.02(17)$ \\
C1-C2-C3 & $122.48(16)$ & C4-C3-C12 & $118.76(16)$ \\
C4-C3-C2 & $123.14(16)$ & C12-C3-C2 & $117.88(17)$ \\
C3-C4-C5 & $119.02(17)$ & C3-C4-C14 & $124.13(17)$ \\
C5-C4-C14 & $116.78(18)$ & C6-C5-C4 & $122.3(2)$ \\
C6-C5-H5 & 118.8 & C4-C5-H5 & 118.8 \\
C5-C6-C7 & $120.23(17)$ & C5-C6-H6 & 119.9 \\
C7-C6-H6 & 119.9 & C6-C7-C8 & $122.34(17)$ \\
C6-C7-C12 & $118.45(17)$ & C8-C7-C12 & $119.14(19)$ \\
C9-C8-C7 & $121.18(17)$ & C9-C8-H8 & 119.4 \\
C7-C8-H8 & 119.4 & C8-C9-C10 & $120.51(18)$ \\
C8-C9-H9 & 119.7 & C10-C9-H9 & 119.7 \\
C11-C10-C9 & $119.7(2)$ & C11-C10-H10 & 120.1 \\
\hline
\end{tabular}




$\begin{array}{llll}\text { C9-C10-H10 } & 120.1 & \text { C10-C11-C12 } & 121.54(17) \\ \text { C10-C11-S1 } & 117.38(15) & \text { C12-C11-S1 } & 120.35(13) \\ \text { C11-C12-C7 } & 117.58(16) & \text { C11-C12-C3 } & 122.62(16) \\ \text { C7-C12-C3 } & 119.70(18) & \text { C1-C13-C2 } & 121.97(17) \\ \text { C1-C13-H13 } & 119.0 & \text { C2-C13-H13 } & 119.0 \\ \text { C4-C14-H14A } & 109.5 & \text { C4-C14-H14B } & 109.5 \\ \text { H14A-C14-H14B } & 109.5 & \text { C4-C14-H14C } & 109.5 \\ \text { H14A-C14-H14C } & 109.5 & \text { H14B-C14-H14C } & 109.5\end{array}$

Table S8. Torsion angles $\left({ }^{\circ}\right)$ for $\mathbf{5 a}$.

\begin{tabular}{|c|c|c|c|}
\hline C11-S1-C1-C13 & $149.18(14)$ & C11-S1-C1-C2 & $-30.93(16)$ \\
\hline C13-C1-C2-C13 & $-2.5(3)$ & $\mathrm{S} 1-\mathrm{C} 1-\mathrm{C} 2-\mathrm{C} 13$ & $177.65(13)$ \\
\hline $\mathrm{C} 13-\mathrm{C} 1-\mathrm{C} 2-\mathrm{C} 3$ & $-177.09(16)$ & $\mathrm{S} 1-\mathrm{C} 1-\mathrm{C} 2-\mathrm{C} 3$ & $3.0(2)$ \\
\hline $\mathrm{C} 13-\mathrm{C} 2-\mathrm{C} 3-\mathrm{C} 4$ & $28.4(3)$ & $\mathrm{C} 1-\mathrm{C} 2-\mathrm{C} 3-\mathrm{C} 4$ & $-157.27(17)$ \\
\hline C13-C2-C3-C12 & $-146.26(17)$ & $\mathrm{C} 1-\mathrm{C} 2-\mathrm{C} 3-\mathrm{C} 12$ & $28.1(2)$ \\
\hline $\mathrm{C} 12-\mathrm{C} 3-\mathrm{C} 4-\mathrm{C} 5$ & $11.6(2)$ & $\mathrm{C} 2-\mathrm{C} 3-\mathrm{C} 4-\mathrm{C} 5$ & $-162.94(16)$ \\
\hline C12-C3-C4-C14 & $-165.34(16)$ & $\mathrm{C} 2-\mathrm{C} 3-\mathrm{C} 4-\mathrm{C} 14$ & $20.1(3)$ \\
\hline C3-C4-C5-C6 & $-1.5(3)$ & C14-C4-C5-C6 & $175.72(17)$ \\
\hline C4-C5-C6-C7 & $-6.9(3)$ & C5-C6-C7-C8 & $-172.31(17)$ \\
\hline C5-C6-C7-C12 & $4.6(3)$ & $\mathrm{C} 6-\mathrm{C} 7-\mathrm{C} 8-\mathrm{C} 9$ & $174.79(18)$ \\
\hline $\mathrm{C} 12-\mathrm{C} 7-\mathrm{C} 8-\mathrm{C} 9$ & $-2.1(3)$ & C7-C8-C9-C10 & $-1.9(3)$ \\
\hline C8-C9-C10-C11 & $1.6(3)$ & C9-C10-C11-C12 & $2.7(3)$ \\
\hline C9-C10-C11-S1 & $-167.48(14)$ & $\mathrm{C} 1-\mathrm{S} 1-\mathrm{C} 11-\mathrm{C} 10$ & $-153.65(15)$ \\
\hline C1-S1-C11-C12 & $36.01(16)$ & $\mathrm{C} 10-\mathrm{C} 11-\mathrm{C} 12-\mathrm{C} 7$ & $-6.5(3)$ \\
\hline $\mathrm{S} 1-\mathrm{C} 11-\mathrm{C} 12-\mathrm{C} 7$ & $163.41(13)$ & $\mathrm{C} 10-\mathrm{C} 11-\mathrm{C} 12-\mathrm{C} 3$ & $177.09(16)$ \\
\hline S1-C11-C12-C3 & $-13.0(2)$ & C6-C7-C12-C11 & $-170.88(16)$ \\
\hline
\end{tabular}




$\begin{array}{llll}\text { C8-C7-C12-C11 } & 6.1(2) & \text { C6-C7-C12-C3 } & 5.6(2) \\ \text { C8-C7-C12-C3 } & -177.39(16) & \text { C4-C3-C12-C11 } & 162.58(17) \\ \text { C2-C3-C12-C11 } & -22.6(2) & \text { C4-C3-C12-C7 } & -13.7(2) \\ \text { C2-C3-C12-C7 } & 161.12(15) & \text { C2-C1-C13-C2 } & 2.6(3) \\ \text { S1-C1-C13-C2 } & -177.51(14) & & \end{array}$

Table S9 Anisotropic atomic displacement parameters $\left(\AA^{2}\right)$ for $\mathbf{5 a}$.

The anisotropic atomic displacement factor exponent takes the form: $-2 \pi^{2}\left[h^{2} a^{* 2} U_{11}\right.$ $\left.+\ldots+2 h k a^{*} b^{*} U_{12}\right]$
$\mathrm{U}_{11}$
$\mathbf{U}_{22}$
$\mathbf{U}_{33}$
$\mathbf{U}_{23}$
$\mathbf{U}_{13}$
$\mathrm{U}_{12}$

$\begin{array}{lllllll}\text { S1 } & 0.0189(2) & 0.0249(3) & 0.0161(2) & -0.00119(18) & 0.00365(15) & 0.00728(18) \\ \text { C1 } & 0.0157(8) & 0.0212(11) & 0.0145(8) & 0.0031(7) & -0.0003(6) & 0.0021(7) \\ \text { C2 } & 0.0166(8) & 0.0192(10) & 0.0123(8) & 0.0009(7) & 0.0001(6) & -0.0006(7) \\ \text { C3 } & 0.0147(8) & 0.0222(11) & 0.0140(8) & -0.0010(7) & 0.0016(6) & -0.0010(7) \\ \text { C4 } & 0.0159(8) & 0.0247(12) & 0.0206(9) & -0.0035(7) & 0.0014(7) & -0.0003(7) \\ \text { C5 } & 0.0194(9) & 0.0266(12) & 0.0260(10) & -0.0097(8) & 0.0024(7) & -0.0014(8) \\ \text { C6 } & 0.0191(9) & 0.0371(13) & 0.0182(9) & -0.0094(8) & 0.0017(7) & -0.0047(8) \\ \text { C7 } & 0.0138(8) & 0.0344(13) & 0.0165(8) & -0.0017(8) & 0.0002(7) & -0.0027(7) \\ \text { C8 } & 0.0165(9) & 0.0459(14) & 0.0132(8) & 0.0017(8) & -0.0023(7) & -0.0019(8) \\ \text { C9 } & 0.0163(9) & 0.0461(15) & 0.0169(9) & 0.0097(8) & -0.0024(7) & 0.0010(8) \\ \text { C10 } & 0.0163(9) & 0.0293(12) & 0.0219(9) & 0.0054(8) & -0.0007(7) & 0.0017(8) \\ \text { C11 } & 0.0135(8) & 0.0274(12) & 0.0152(8) & 0.0023(7) & -0.0019(6) & -0.0009(7) \\ \text { C12 } & 0.0128(8) & 0.0259(11) & 0.0143(8) & 0.0012(7) & -0.0006(6) & -0.0035(7) \\ \text { C13 } & 0.0187(9) & 0.0164(10) & 0.0155(8) & 0.0004(7) & 0.0017(6) & 0.0022(7) \\ \text { C14 } & 0.0283(10) & 0.0202(12) & 0.0300(10) & -0.0023(8) & -0.0012(8) & 0.0021(8)\end{array}$


Table S10. Hydrogen atomic coordinates and isotropic atomic displacement parameters $\left(\AA^{2}\right)$ for $\mathbf{5 a}$.

\begin{tabular}{lcccc}
\hline & $\mathbf{x} / \mathbf{a}$ & $\mathbf{y} / \mathbf{b}$ & $\mathbf{z} / \mathbf{c}$ & $\mathbf{U}(\mathbf{e q})$ \\
H5 & 0.3224 & 0.0211 & 0.2093 & 0.029 \\
H6 & 0.2374 & 0.1942 & 0.0920 & 0.03 \\
H8 & 0.1158 & 0.4617 & 0.0450 & 0.03 \\
H9 & 0.0403 & 0.7163 & 0.0845 & 0.032 \\
H10 & 0.0784 & 0.8054 & 0.2402 & 0.027 \\
H13 & 0.3927 & 0.7330 & 0.5606 & 0.02 \\
H14A & 0.5168 & 0.0315 & 0.3824 & 0.039 \\
H14B & 0.3329 & -0.0369 & 0.3758 & 0.039 \\
H14C & 0.3854 & 0.1001 & 0.4499 & 0.039
\end{tabular}




\section{References}

[1] Gaussian 09; Revision D.01; Frisch, M. J.; Trucks, G. W.; Schlegel, H. B.; Scuseria, G. E.;

Robb, M. A.; Cheeseman, J. R.; Scalmani, G.; Barone, V.; Mennucci, B.; Petersson, G. A.; Nakatsuji, H.; Caricato, M.; Li, X.; Hratchian, H. P.; Izmaylov, A. F.; Bloino, J.; Zheng, G.; Sonnenberg, J. L.; Hada, M.; Ehara, M.; Toyota, K.; Fukuda, R.; Hasegawa, J.; Ishida, M.; Nakajima, T.; Honda, Y.; Kitao, O.; Nakai, H.; Vreven, T.; Montgomery, J. A.; Jr. Peralta, J. E.; Ogliaro, F.; Bearpark, M.; Heyd, J. J.; Brothers, E.; Kudin, K. N.; Staroverov, V. N.; Kobayashi, R.; Normand, J.; Raghavachari, K.; Rendell, A.; Burant, J. C.; Iyengar, S. S.; Tomasi, J.; Cossi, M.; Rega, N.; Millam, J. M.; Klene, M.; Knox, J. E.; Cross, J. B.; Bakken, V.; Adamo, C.; Jaramillo, J.; Gomperts, R.; Stratmann, R. E.; Yazyev, O.; Austin, A. J.; Cammi, R.; Pomelli, C.; Ochterski, J. W.; Martin, R. L.; Morokuma, K.; Zakrzewski, V. G.; Voth, G. A.; Salvador, P.; Dannenberg, J. J.; Dapprich, S.; Daniels, A. D.; Farkas, Ö.: Foresman, J. B.; Ortiz, J. V.; Cioslowski, J.; Fox, D. J., Gaussian, Inc., Wallingford CT, 2009.

[2] (a) Becke, A. D. A New Mixing of Hartree-Fock and Local Density-Functional Theories. J. Chem. Phys. 1993, 98, 1372-1377; (b) Lee, C.; Yang, W.; Parr, R. G. Development of The Colle-Salvetti Correlation-Energy Formula into a Functional of the Electron Density. Phys. Rev. B: Condens. Matter 1988, 37, 785-789; (c) Yanai, T.; Tew, D.; Handy, N. A New Hybrid Exchange-Correlation Functional using the Coulomb-Attenuating Method (CAM-B3LYP). Chem. Phys. Lett. 2004, 393, 51-57; (d) Ditchfield, R.; Hehre, W. J.; Pople, J. A. SelfConsistent Molecular-Orbital Methods. IX. An Extended Gaussian-Type Basis for Molecular-Orbital Studies of Organic Molecules. J. Chem. Phys. 1971, 54, 724-728; (e) Hehre, W. J.; Ditchfield, R.; Pople, J. A. Self-Consistent Molecular-Orbital Methods. XII. Further Extensions of Gaussian-Type Basis Sets for Use in Molecular-Orbital Studies of Organic Molecules. J. Chem. Phys. 1972, 56, 2257-2261. (f) Hariharan, P. C.; Pople, J. A. The Influence of Polarization Functions on Molecular Orbital Hydrogenation Energies. Theor. Chim. Acta 1973, 28, 213-222.

[3] (a) Yamanaka, S.; Okumura, M.; Nakano, M.; Yamaguchi, K. EHF Theory of Chemical Reactions Part 4. UNO CASSCF, UNO CASPT2 and R(U)HF Coupled-Cluster (CC) Wavefunctions. J. Mol. Struct. 1994, 310, 205-218; (b) Kamada, K.; Ohta, K.; Shimizu, A.; Kubo, T.; Kishi, R.; Takahashi, H.; Botek, E.; Champagne, B.; Nakano, M. Singlet Diradical Character from Experiment. J. Phys. Chem. Lett. 2010, 1, 937-940. 


\section{Appendix: Cartesian coordinates and other computational results}

Table S11. Cartesian coordinates for optimized structure HZ-2S.

\begin{tabular}{|c|c|c|c|c|}
\hline No. & Atom No. & $X$ & $\mathrm{Y}$ & $\mathrm{Z}$ \\
\hline 1 & 6 & -3.208562 & 2.314899 & 0.000463 \\
\hline 2 & 6 & -4.571159 & 2.659076 & 0.000429 \\
\hline 3 & 6 & -2.823686 & 0.925391 & 0.000141 \\
\hline 4 & 6 & -3.891273 & -0.057188 & -0.000087 \\
\hline 5 & 6 & -5.269005 & 0.350791 & -0.000124 \\
\hline 6 & 6 & -5.581202 & 1.722425 & 0.000116 \\
\hline 7 & 6 & -3.695849 & -1.469721 & -0.000263 \\
\hline 8 & 6 & -4.764073 & -2.392661 & -0.000491 \\
\hline 9 & 6 & -6.081297 & -1.957459 & -0.000560 \\
\hline 10 & 6 & -6.327188 & -0.590989 & -0.000372 \\
\hline 11 & 6 & -1.415559 & 0.479112 & 0.000062 \\
\hline 12 & 6 & -1.029578 & -0.905977 & -0.000003 \\
\hline 13 & 16 & -2.146288 & -2.224383 & -0.000165 \\
\hline 14 & 6 & -0.304983 & 1.333444 & -0.000015 \\
\hline 15 & 6 & 1.029587 & 0.905989 & -0.000035 \\
\hline 16 & 6 & 1.415565 & -0.479099 & 0.000066 \\
\hline 17 & 6 & 0.304992 & -1.333432 & 0.000025 \\
\hline 18 & 6 & -2.291355 & 3.518486 & 0.000913 \\
\hline 19 & 16 & 2.146305 & 2.224388 & -0.000240 \\
\hline 20 & 6 & 3.695863 & 1.469720 & -0.000279 \\
\hline 21 & 6 & 3.891279 & 0.057186 & -0.000078 \\
\hline 22 & 6 & 2.823686 & -0.925382 & 0.000139 \\
\hline 23 & 6 & 4.764095 & 2.392652 & -0.000499 \\
\hline 24 & 6 & 6.081316 & 1.957439 & -0.000531 \\
\hline 25 & 6 & 6.327197 & 0.590966 & -0.000325 \\
\hline 26 & 6 & 5.269006 & -0.350806 & -0.000093 \\
\hline 27 & 6 & 5.581188 & -1.722445 & 0.000145 \\
\hline 28 & 6 & 4.571135 & -2.659086 & 0.000419 \\
\hline 29 & 6 & 3.208542 & -2.314892 & 0.000437 \\
\hline 30 & 6 & 2.291301 & -3.518455 & 0.000829 \\
\hline 31 & 1 & -4.835081 & 3.710504 & 0.000662 \\
\hline 32 & 1 & -6.620542 & 2.035905 & 0.000090 \\
\hline 33 & 1 & -4.544593 & -3.456512 & -0.000613 \\
\hline 34 & 1 & -6.896332 & -2.672209 & -0.000741 \\
\hline 35 & 1 & -7.347133 & -0.219251 & -0.000401 \\
\hline 36 & 1 & -0.446798 & 2.396490 & -0.000099 \\
\hline 37 & 1 & 0.446811 & -2.396480 & -0.000025 \\
\hline 38 & 1 & -2.887886 & 4.430659 & 0.001420 \\
\hline 39 & 1 & -1.657384 & 3.560628 & -0.890938 \\
\hline 40 & 1 & -1.657135 & 3.559775 & 0.892626 \\
\hline 41 & 1 & 4.544624 & 3.456505 & -0.000642 \\
\hline 42 & 1 & 6.896356 & 2.672182 & -0.000703 \\
\hline 43 & 1 & 7.347139 & 0.219220 & -0.000332 \\
\hline 44 & 1 & 6.620525 & -2.035936 & 0.000137 \\
\hline
\end{tabular}




$\begin{array}{rrrrr}45 & 1 & 4.835046 & -3.710517 & 0.000637 \\ 46 & 1 & 2.887804 & -4.430647 & 0.001204 \\ 47 & 1 & 1.657263 & -3.560484 & -0.890980 \\ 48 & 1 & 1.657142 & -3.559815 & 0.892582\end{array}$

Table S12. Cartesian coordinates for optimized structure OZ-2S.

\begin{tabular}{ccccc} 
No. & Atom No. & X & Y & Z \\
\hline 1 & 6 & 4.305852 & -2.291651 & 0.000601 \\
2 & 6 & 5.650963 & -2.702997 & 0.000481 \\
3 & 6 & 3.984971 & -0.887154 & 0.000209 \\
4 & 6 & 5.100970 & 0.038136 & -0.000128 \\
5 & 6 & 6.458563 & -0.437008 & -0.000238 \\
6 & 6 & 6.706590 & -1.820632 & 0.000043 \\
7 & 6 & 4.980442 & 1.462139 & -0.000353 \\
8 & 6 & 6.096565 & 2.329639 & -0.000689 \\
9 & 6 & 7.388679 & 1.828614 & -0.000827 \\
10 & 6 & 7.562547 & 0.450702 & -0.000598 \\
11 & 6 & 2.588496 & -0.376078 & 0.000136 \\
12 & 6 & 2.284312 & 1.048621 & 0.000038 \\
13 & 16 & 3.481029 & 2.300518 & -0.000195 \\
14 & 6 & 1.455749 & -1.190830 & 0.000101 \\
15 & 6 & 0.128216 & -0.702020 & 0.000094 \\
16 & 6 & -0.128212 & 0.702023 & 0.000107 \\
17 & 6 & 0.987697 & 1.552665 & 0.000054 \\
18 & 6 & -0.987694 & -1.552661 & 0.000032 \\
19 & 6 & -2.284309 & -1.048616 & 0.000031 \\
20 & 6 & -2.588494 & 0.376082 & 0.000152 \\
21 & 6 & -1.455746 & 1.190832 & 0.000126 \\
22 & 16 & -3.481023 & -2.300516 & -0.000212 \\
23 & 6 & -4.980436 & -1.462140 & -0.000345 \\
24 & 6 & -5.100969 & -0.038137 & -0.000113 \\
25 & 6 & -3.984971 & 0.887157 & 0.000224 \\
26 & 6 & -6.096557 & -2.329643 & -0.000682 \\
27 & 6 & -7.388673 & -1.828623 & -0.000815 \\
28 & 6 & -7.562544 & -0.450712 & -0.000587 \\
29 & 6 & -6.458563 & 0.437002 & -0.000230 \\
30 & 6 & -6.706596 & 1.820624 & 0.000034 \\
31 & 6 & -5.650973 & 2.702993 & 0.000459 \\
32 & 6 & -4.305861 & 2.291653 & 0.000595 \\
33 & 6 & -3.337337 & 3.454863 & 0.001206 \\
34 & 6 & 3.337317 & -3.454851 & 0.001218 \\
35 & 1 & 5.860718 & -3.766535 & 0.000761 \\
36 & 1 & 7.729417 & -2.183730 & -0.000038 \\
37 & 1 & 5.929886 & 3.402958 & -0.000843 \\
38 & 1 & 8.239885 & 2.499848 & -0.001094 \\
39 & 1 & 8.561943 & 0.026850 & -0.000679 \\
40 & 1 & 1.547694 & -2.257796 & 0.000047 \\
41 & 1 & 0.836803 & 2.628750 & -0.000007
\end{tabular}




$\begin{array}{llrrr}42 & 1 & -0.836800 & -2.628747 & -0.000045 \\ 43 & 1 & -1.547688 & 2.257798 & 0.000088 \\ 44 & 1 & -5.929875 & -3.402962 & -0.000844 \\ 45 & 1 & -8.239876 & -2.499859 & -0.001083 \\ 46 & 1 & -8.561942 & -0.026862 & -0.000671 \\ 47 & 1 & -7.729425 & 2.183718 & -0.000053 \\ 48 & 1 & -5.860732 & 3.766531 & 0.000725 \\ 49 & 1 & -3.894689 & 4.391620 & 0.001502 \\ 50 & 1 & -2.700749 & 3.468039 & -0.889363 \\ 51 & 1 & -2.701000 & 3.467327 & 0.891960 \\ 52 & 1 & 2.700830 & -3.468111 & -0.889423 \\ 53 & 1 & 2.700876 & -3.467213 & 0.891900 \\ 54 & 1 & 3.894657 & -4.391614 & 0.001668\end{array}$

Table S13. Cartesian coordinates for optimized structure DBHZ-2S.

Total Energy: -2800.42942020 hartrees (singlet)

Total Energy: -2800.42506728 hartrees (triplet)

\begin{tabular}{|c|c|c|c|c|}
\hline No. & Atom No. & $X$ & $\mathrm{Y}$ & $\mathrm{Z}$ \\
\hline 1 & 6 & -5.102891 & 3.634389 & -0.939717 \\
\hline 2 & 6 & -3.746031 & 3.587891 & -1.306024 \\
\hline 3 & 6 & -3.016724 & 2.417171 & -1.166485 \\
\hline 4 & 6 & -3.611192 & 1.229312 & -0.675064 \\
\hline 5 & 6 & -5.031650 & 1.246574 & -0.434901 \\
\hline 6 & 6 & -5.734060 & 2.480078 & -0.521085 \\
\hline 7 & 6 & -2.870744 & 0.004139 & -0.478878 \\
\hline 8 & 6 & -3.606473 & -1.220867 & -0.452614 \\
\hline 9 & 6 & -5.037845 & -1.198572 & -0.264050 \\
\hline 10 & 6 & -5.735946 & 0.039948 & -0.169825 \\
\hline 11 & 6 & -2.992573 & -2.495193 & -0.614753 \\
\hline 12 & 6 & -3.741863 & -3.677887 & -0.590803 \\
\hline 13 & 6 & -5.117837 & -3.638469 & -0.367828 \\
\hline 14 & 6 & -5.755322 & -2.417885 & -0.203083 \\
\hline 15 & 6 & -7.197682 & 0.058793 & 0.133021 \\
\hline 16 & 6 & -7.620302 & 0.017527 & 1.481823 \\
\hline 17 & 6 & -8.990945 & 0.040138 & 1.750881 \\
\hline 18 & 6 & -9.949573 & 0.092762 & 0.733633 \\
\hline 19 & 6 & -9.500484 & 0.119994 & -0.591332 \\
\hline 20 & 6 & -8.142126 & 0.110775 & -0.918970 \\
\hline 21 & 6 & -1.435591 & -0.001324 & -0.233743 \\
\hline 22 & 6 & -0.625119 & -1.161332 & -0.452845 \\
\hline 23 & 16 & -1.293340 & -2.686654 & -0.985027 \\
\hline 24 & 6 & -0.755556 & 1.128005 & 0.262633 \\
\hline 25 & 6 & 0.623194 & 1.160566 & 0.445637 \\
\hline 26 & 6 & 1.433776 & 0.000718 & 0.226470 \\
\hline 27 & 6 & 0.753645 & -1.128866 & -0.269576 \\
\hline 28 & 16 & 1.291142 & 2.687016 & 0.974721 \\
\hline 29 & 6 & 2.989555 & 2.495675 & 0.600523 \\
\hline 30 & 6 & 3.604427 & 1.220987 & 0.444419 \\
\hline
\end{tabular}




$\begin{array}{llccc}31 & 6 & 2.868985 & -0.004341 & 0.470982 \\ 32 & 6 & 3.736554 & 3.679353 & 0.563379 \\ 33 & 6 & 5.109858 & 3.640959 & 0.323676 \\ 34 & 6 & 5.747587 & 2.420427 & 0.160778 \\ 35 & 6 & 5.035576 & 1.199718 & 0.252470 \\ 36 & 6 & 5.735061 & -0.038018 & 0.166411 \\ 37 & 6 & 5.031285 & -1.244523 & 0.433716 \\ 38 & 6 & 3.609953 & -1.229288 & 0.667852 \\ 39 & 6 & 5.741102 & -2.471296 & 0.548647 \\ 40 & 6 & 5.110667 & -3.625415 & 0.969354 \\ 41 & 6 & 3.749318 & -3.582525 & 1.318098 \\ 42 & 6 & 3.016613 & -2.415468 & 1.164113 \\ 43 & 6 & 7.199229 & -0.059824 & -0.127823 \\ 44 & 6 & 7.629104 & -0.201575 & -1.466823 \\ 45 & 6 & 9.001481 & -0.209956 & -1.727812 \\ 46 & 6 & 9.954422 & -0.090546 & -0.710685 \\ 47 & 6 & 9.497595 & 0.046268 & 0.604305 \\ 48 & 6 & 8.136884 & 0.063919 & 0.923323 \\ 49 & 6 & 6.642553 & -0.333973 & -2.605911 \\ 50 & 6 & 7.703133 & 0.205034 & 2.365813 \\ 51 & 6 & 11.429842 & -0.133825 & -1.020111 \\ 52 & 6 & -7.718953 & 0.134707 & -2.371405 \\ 53 & 6 & -6.628726 & -0.031118 & 2.623220 \\ 54 & 6 & -11.422165 & 0.140122 & 1.054292 \\ 55 & 1 & -5.658586 & 4.562413 & -1.020333 \\ 56 & 1 & -3.264134 & 4.472398 & -1.710012 \\ 57 & 1 & -1.985954 & 2.404577 & -1.497015 \\ 58 & 1 & -6.791148 & 2.488123 & -0.283231 \\ 59 & 1 & -3.243612 & -4.631030 & -0.739586 \\ 60 & 1 & -5.685146 & -4.561982 & -0.331945 \\ 61 & 1 & -6.826281 & -2.378111 & -0.044696 \\ 62 & 1 & -9.318209 & 0.015215 & 2.786996 \\ 63 & 1 & -10.229200 & 0.144366 & -1.397340 \\ 64 & 1 & -1.323068 & 2.014267 & 0.513487 \\ 65 & 1 & 1.321276 & -2.015061 & -0.520528 \\ 66 & 1 & 3.237643 & 4.632718 & 0.708418 \\ 67 & 1 & 5.673914 & 4.565513 & 0.268506 \\ 68 & 1 & 6.814577 & 2.381761 & -0.022710 \\ 69 & 1 & 6.803236 & -2.472638 & 0.334068 \\ 70 & 1 & 5.671101 & -4.548663 & 1.069498 \\ 71 & 1 & 3.267329 & -4.465496 & 1.725347 \\ 72 & 1 & 1.984175 & -2.404020 & 1.489388 \\ 73 & 1 & 9.334546 & -0.310352 & -2.757550 \\ 74 & 1 & 10.221288 & 0.142125 & 1.409477 \\ 75 & 1 & 6.009885 & -1.222372 & -2.496486 \\ 76 & 1 & 7.164022 & -0.419624 & -3.561107 \\ 77 & 1 & 5.974788 & 0.532746 & -2.672562 \\ 78 & 1 & 8.570859 & 0.272065 & 3.024642 \\ 79 & 1 & 7.104075 & -0.650312 & 2.698035 \\ 80 & 1 & 7.097766 & 1.103776 & 2.529091\end{array}$




$\begin{array}{lllrl}81 & 1 & 11.642456 & 0.266172 & -2.014933 \\ 82 & 1 & 11.800458 & -1.165580 & -0.997436 \\ 83 & 1 & 12.009666 & 0.436260 & -0.289691 \\ 84 & 1 & -8.587707 & 0.051507 & -3.027089 \\ 85 & 1 & -7.040768 & -0.690078 & -2.616417 \\ 86 & 1 & -7.201193 & 1.064573 & -2.632754 \\ 87 & 1 & -6.009823 & -0.935128 & 2.591255 \\ 88 & 1 & -7.145708 & -0.023024 & 3.584605 \\ 89 & 1 & -5.948133 & 0.827874 & 2.608325 \\ 90 & 1 & -11.639011 & -0.331791 & 2.015931 \\ 91 & 1 & -12.015922 & -0.359728 & 0.284443 \\ 92 & 1 & -11.772461 & 1.177606 & 1.112866\end{array}$

Table S14. Mulliken charges and spin densities for optimized structure DBHZ-2S.
1 C $-0.076787-0.007667$
2 C $-0.071132-0.028280$
3 C $-0.151703-0.053893$
$\begin{array}{llll}4 & \mathrm{C} & 0.059285 & 0.023863\end{array}$
$\begin{array}{llll}5 & \mathrm{C} & 0.095786 & 0.044994\end{array}$
6 C $-0.113327-0.078162$
7 C $-0.018349-0.176660$
8 C $0.101406 \quad 0.038966$
9 C $0.106016 \quad 0.055193$
10 C $-0.019675-0.201775$
$\begin{array}{llll}11 & \mathrm{C} & -0.187040 & -0.057443\end{array}$
12 C $-0.085292-0.073255$
$\begin{array}{lllll}13 & \mathrm{C} & -0.068107 & 0.004241\end{array}$
$14 \mathrm{C}-0.109542-0.147103$
$\begin{array}{llll}15 & \mathrm{C} & -0.160467 & 0.016521\end{array}$
$\begin{array}{llll}16 \mathrm{C} & 0.147017 & -0.008159\end{array}$
$\begin{array}{llll}17 & \mathrm{C} & -0.176402 & 0.001090\end{array}$
$18 \mathrm{C} \quad 0.152044-0.003513$
19 C $-0.175311-0.000103$
$20 \mathrm{C} \quad 0.139365-0.006342$
$\begin{array}{llll}21 & \mathrm{C} & 0.127768 & 0.050909\end{array}$
$\begin{array}{llll}22 & \mathrm{C} & -0.168142 & -0.041118\end{array}$
$23 \mathrm{~S} \quad 0.352541-0.115605$
$24 \mathrm{C} \quad-0.145215 \quad-0.032033$
$\begin{array}{llll}25 & \mathrm{C} & -0.168280 & 0.041747\end{array}$
$\begin{array}{llll}26 & \mathrm{C} & 0.127974 & -0.050194\end{array}$
$\begin{array}{llll}27 & \mathrm{C} & -0.145443 & 0.031430\end{array}$
$\begin{array}{llll}28 \mathrm{~S} & 0.352332 & 0.116195\end{array}$
$\begin{array}{llll}29 & \mathrm{C} & -0.187381 & 0.057288\end{array}$
$30 \mathrm{C} \quad 0.101536-0.038551$
$\begin{array}{llll}31 & \mathrm{C} & -0.017845 & 0.175817\end{array}$
$\begin{array}{llll}32 & \mathrm{C} & -0.084908 & 0.074232\end{array}$
$\begin{array}{llll}33 \mathrm{C} & -0.068443 & -0.004705\end{array}$
$\begin{array}{llll}34 \mathrm{C} & -0.110686 & 0.148509\end{array}$
$\begin{array}{llll}35 & \mathrm{C} & 0.108597 & -0.056309\end{array}$ 


\begin{tabular}{|c|c|c|}
\hline & & \\
\hline & & \\
\hline $8 \mathrm{C}$ & & \\
\hline C & -0.1 & \\
\hline ) & -0.076207 & 0.00669 \\
\hline & $-0.0^{\prime}$ & \\
\hline & -0.1 & \\
\hline & -0.1 & -0 . \\
\hline & & 0.0 \\
\hline & -0.1 & -0. \\
\hline & & \\
\hline & -0.1 & -0 . \\
\hline & 0.1 & \\
\hline & -0 . & \\
\hline $0 \mathrm{C}$ & -0. & \\
\hline & -0.3 & -0 \\
\hline & -0. & \\
\hline & -0.3 & \\
\hline & -0.3 & \\
\hline & 0.1 & \\
\hline & & \\
\hline & & \\
\hline & & \\
\hline & 0.1 & \\
\hline & & \\
\hline & & \\
\hline & 0.1 & -0. \\
\hline & & \\
\hline & & \\
\hline & & \\
\hline & & -0. \\
\hline & & \\
\hline & & -0. \\
\hline & & -0. \\
\hline & & \\
\hline & & \\
\hline & & \\
\hline & & \\
\hline & & \\
\hline & 0.1 & \\
\hline & 0.1 & \\
\hline & & -0.0 \\
\hline 1011 & & -0. \\
\hline 79 & 0.1 & 0.0 \\
\hline & 0.1 & 0.000 \\
\hline & & \\
\hline & 0.14 & 0.00 \\
\hline & 747 & 0.000 \\
\hline & & \\
\hline & ${ }_{1}$ & 0.00 \\
\hline
\end{tabular}


$86 \mathrm{H} \quad 0.125665-0.000250$

$87 \mathrm{H} \quad 0.118064-0.000235$

$88 \mathrm{H} \quad 0.144061-0.000074$

$\begin{array}{llll}89 & \mathrm{H} & 0.119851 & 0.000053\end{array}$

$90 \mathrm{H} \quad 0.134052-0.000078$

$\begin{array}{lllll}91 \mathrm{H} & 0.136190 & -0.000039\end{array}$

$92 \mathrm{H} \quad 0.150210-0.000285$

Sum of Mulliken charges $=2.00000 \quad 0.00000$

Table S15. Cartesian coordinates for optimized structure DBOZ-2S.

Total Energy: -2954.08492746 hartrees (singlet)

Total Energy: -2954.08292529 hartrees (triplet)

\begin{tabular}{ccccc} 
No. & Atom No. & $\mathrm{X}$ & $\mathrm{Y}$ & $\mathrm{Z}$ \\
\hline 1 & 6 & 6.050651 & 2.887636 & -2.163058 \\
2 & 6 & 4.739397 & 2.587957 & -2.572676 \\
3 & 6 & 4.063688 & 1.503881 & -2.035229 \\
4 & 6 & 4.669569 & 0.655690 & -1.075156 \\
5 & 6 & 6.051720 & 0.897014 & -0.749372 \\
6 & 6 & 6.696210 & 2.049471 & -1.275185 \\
7 & 6 & 3.981519 & -0.465371 & -0.481116 \\
8 & 6 & 4.771211 & -1.499775 & 0.101812 \\
9 & 6 & 6.165096 & -1.263163 & 0.400969 \\
10 & 6 & 6.783192 & -0.033182 & 0.040509 \\
11 & 6 & 4.247188 & -2.789697 & 0.409029 \\
12 & 6 & 5.044911 & -3.779443 & 0.999569 \\
13 & 6 & 6.371322 & -3.512360 & 1.333679 \\
14 & 6 & 6.922443 & -2.272155 & 1.042876 \\
15 & 6 & 8.199058 & 0.246779 & 0.427930 \\
16 & 6 & 9.258647 & -0.142772 & -0.423862 \\
17 & 6 & 10.568403 & 0.138309 & -0.026680 \\
18 & 6 & 10.859859 & 0.791923 & 1.175689 \\
19 & 6 & 9.790739 & 1.162248 & 1.997102 \\
20 & 6 & 8.461517 & 0.904578 & 1.650079 \\
21 & 6 & 2.522221 & -0.515920 & -0.407989 \\
22 & 6 & 1.808818 & -1.765096 & -0.264697 \\
23 & 16 & 2.639604 & -3.294341 & -0.049368 \\
24 & 6 & 1.751532 & 0.650841 & -0.386123 \\
25 & 6 & 0.342799 & 0.630754 & -0.362639 \\
26 & 6 & -0.342792 & -0.630787 & -0.362632 \\
27 & 6 & 0.428387 & -1.812811 & -0.279664 \\
28 & 6 & 7.343807 & 1.326338 & 2.577832 \\
29 & 6 & 9.005350 & -0.851081 & -1.736573 \\
30 & 6 & 12.284082 & 1.106420 & 1.560070 \\
31 & 6 & -0.428381 & 1.812780 & -0.279688 \\
32 & 6 & -1.808810 & 1.765065 & -0.264727 \\
33 & 6 & -2.522215 & 0.515886 & -0.408000 \\
34 & 6 & -1.751525 & -0.650873 & -0.386119 \\
35 & 16 & -2.639595 & 3.294318 & -0.049444
\end{tabular}




$\begin{array}{llccc}36 & 6 & -4.247176 & 2.789683 & 0.408982 \\ 37 & 6 & -4.771200 & 1.499757 & 0.101793 \\ 38 & 6 & -3.981513 & 0.465337 & -0.481117 \\ 39 & 6 & -5.044895 & 3.779443 & 0.999505 \\ 40 & 6 & -6.371305 & 3.512369 & 1.333626 \\ 41 & 6 & -6.922429 & 2.272160 & 1.042847 \\ 42 & 6 & -6.165086 & 1.263157 & 0.400954 \\ 43 & 6 & -6.783191 & 0.033177 & 0.040508 \\ 44 & 6 & -6.051724 & -0.897038 & -0.749355 \\ 45 & 6 & -4.669570 & -0.655728 & -1.075140 \\ 46 & 6 & -6.696218 & -2.049503 & -1.275151 \\ 47 & 6 & -6.050662 & -2.887684 & -2.163009 \\ 48 & 6 & -4.739406 & -2.588016 & -2.572631 \\ 49 & 6 & -4.063693 & -1.503935 & -2.035202 \\ 50 & 6 & -8.199062 & -0.246761 & 0.427937 \\ 51 & 6 & -8.461534 & -0.904495 & 1.650110 \\ 52 & 6 & -9.790765 & -1.162145 & 1.997138 \\ 53 & 6 & -10.859869 & -0.791842 & 1.175708 \\ 54 & 6 & -10.568399 & -0.138287 & -0.026698 \\ 55 & 6 & -9.258645 & 0.142761 & -0.423887 \\ 56 & 6 & -7.343835 & -1.326233 & 2.577887 \\ 57 & 6 & -9.005321 & 0.850951 & -1.736657 \\ 58 & 6 & -12.284118 & -1.106224 & 1.560105 \\ 59 & 1 & 6.565309 & 3.751664 & -2.569647 \\ 60 & 1 & 4.253970 & 3.201071 & -3.325142 \\ 61 & 1 & 3.071109 & 1.273810 & -2.400560 \\ 62 & 1 & 7.723828 & 2.239825 & -0.989088 \\ 63 & 1 & 4.618947 & -4.756445 & 1.206138 \\ 64 & 1 & 6.971041 & -4.278018 & 1.813589 \\ 65 & 1 & 7.956533 & -2.062868 & 1.289207 \\ 66 & 1 & 11.384991 & -0.163836 & -0.677338 \\ 67 & 1 & 9.994190 & 1.666419 & 2.938214 \\ 68 & 1 & 2.246614 & 1.613219 & -0.354489 \\ 69 & 1 & -0.079615 & -2.768173 & -0.185081 \\ 70 & 1 & 7.742493 & 1.835552 & 3.457367 \\ 71 & 1 & 6.760063 & 0.468294 & 2.930878 \\ 72 & 1 & 6.644160 & 2.013194 & 2.087925 \\ 73 & 1 & 8.397799 & -0.245480 & -2.418475 \\ 74 & 1 & 8.478615 & -1.801675 & -1.595915 \\ 75 & 1 & 9.946449 & -1.070732 & -2.244324 \\ 76 & 1 & 12.965830 & 0.307313 & 1.255964 \\ 77 & 1 & 12.386233 & 1.251954 & 2.638273 \\ 78 & 1 & 12.624379 & 2.026544 & 1.070360 \\ 79 & 1 & 0.079622 & 2.768143 & -0.185115 \\ 80 & 1 & -2.246607 & -1.613251 & -0.354469 \\ 81 & 1 & -4.618927 & 4.756448 & 1.206052 \\ 82 & 1 & -6.971022 & 4.278038 & 1.813522 \\ 83 & 1 & -7.956521 & 2.062881 & 1.289177 \\ 84 & 1 & -7.723838 & -2.239844 & -0.989053 \\ 85 & 1 & -6.565322 & -3.751717 & -2.569585\end{array}$




$\begin{array}{lllll}86 & 1 & -4.253982 & -3.201143 & -3.325089 \\ 87 & 1 & -3.071114 & -1.273871 & -2.400538 \\ 88 & 1 & -9.994221 & -1.666278 & 2.938269 \\ 89 & 1 & -11.384986 & 0.163831 & -0.677370 \\ 90 & 1 & -6.760047 & -0.468187 & 2.930855 \\ 91 & 1 & -6.644224 & -2.013162 & 2.088029 \\ 92 & 1 & -7.742534 & -1.835358 & 3.457467 \\ 93 & 1 & -8.397973 & 0.245171 & -2.418583 \\ 94 & 1 & -8.478356 & 1.801432 & -1.596108 \\ 95 & 1 & -9.946421 & 1.070777 & -2.244330 \\ 96 & 1 & -12.386312 & -1.251494 & 2.638342 \\ 97 & 1 & -12.965819 & -0.307154 & 1.255794 \\ 98 & 1 & -12.624421 & -2.026445 & 1.070590\end{array}$

Table S16. Mulliken charges and spin densities for optimized structure DBOZ-2S.
1 C $-0.076584-0.004933$
$\begin{array}{llll}2 \text { C } & -0.074581 & -0.039628\end{array}$
3 C $-0.142445-0.056647$
$\begin{array}{llll}4 \mathrm{C} & 0.055498 & 0.019581\end{array}$
$\begin{array}{llll}5 \mathrm{C} & 0.093590 & 0.055746\end{array}$
$\begin{array}{llll}6 \text { C } & -0.113993 & -0.094168\end{array}$
$\begin{array}{llll}7 \mathrm{C} & -0.010237 & -0.187041\end{array}$
$\begin{array}{llll}8 \mathrm{C} & 0.096481 & 0.040999\end{array}$
$\begin{array}{llll}9 \mathrm{C} & 0.110957 & 0.067629\end{array}$
10 C $-0.024086-0.243710$
$\begin{array}{llll}11 & \mathrm{C} & -0.182212 & -0.065348\end{array}$
12 C $-0.086409-0.088622$
$\begin{array}{llll}13 & \mathrm{C} & -0.067021 & 0.007383\end{array}$
$14 \mathrm{C}-0.113834-0.171793$
$\begin{array}{lllll}15 & \mathrm{C} & -0.160998 & 0.022081\end{array}$
16 C $0.140190 \quad-0.007380$
$\begin{array}{llll}17 & \mathrm{C} & -0.175693 & 0.000504\end{array}$
18 C $\quad 0.151528-0.000717$
$\begin{array}{llll}19 & \mathrm{C} & -0.177877 & 0.000284\end{array}$
20 C $\quad 0.147524-0.008031$
$\begin{array}{llll}21 & \mathrm{C} & 0.096171 & 0.036198\end{array}$
22 C $-0.162135-0.029576$
$23 \mathrm{~S} \quad 0.347252-0.118672$
$24 \mathrm{C}-0.172848-0.050448$
$\begin{array}{llll}25 & \mathrm{C} & 0.124342 & 0.030688\end{array}$
$26 \mathrm{C} \quad 0.124343 \quad-0.030688$
$\begin{array}{llll}27 & \mathrm{C} & -0.155763 & 0.017122\end{array}$
$\begin{array}{llll}28 & \mathrm{C} & -0.387505 & 0.000290\end{array}$
$\begin{array}{llll}29 & \mathrm{C} & -0.387932 & 0.000161\end{array}$
$\begin{array}{llll}30 & \mathrm{C} & -0.386957 & 0.000034\end{array}$
$\begin{array}{llll}31 & \mathrm{C} & -0.155762 & -0.017122\end{array}$
$\begin{array}{llll}32 & \mathrm{C} & -0.162134 & 0.029576\end{array}$
$\begin{array}{llll}33 & \mathrm{C} & 0.096171 & -0.036199\end{array}$
$\begin{array}{llll}34 & \mathrm{C} & -0.172849 & 0.050449\end{array}$ 


\begin{tabular}{|c|c|c|}
\hline & & \\
\hline & 211 & \\
\hline & & \\
\hline & & \\
\hline & & \\
\hline & -0.0 & \\
\hline & & \\
\hline & $\gamma_{1}$ & \\
\hline & $C \quad-0.0$ & \\
\hline & 0.0 & \\
\hline & & \\
\hline & $C-0$. & \\
\hline & $C \quad-0$. & \\
\hline & $C \quad-0$. & \\
\hline & & \\
\hline & $C \quad-0$. & \\
\hline & 0.1 & \\
\hline & $C \quad-0$. & \\
\hline & & \\
\hline & $C \quad-0$. & \\
\hline & 0.1 & \\
\hline & $C-0$. & \\
\hline & $C-0$ & \\
\hline & -0. & \\
\hline & & \\
\hline & & \\
\hline & $H$ & \\
\hline & 0.1 & \\
\hline & & \\
\hline & & \\
\hline & & \\
\hline & 0.1 & \\
\hline & & \\
\hline & & \\
\hline & & \\
\hline & & \\
\hline & & \\
\hline & & \\
\hline & & \\
\hline & & \\
\hline & $\begin{array}{ll}H & 0.1\end{array}$ & \\
\hline & & \\
\hline & & \\
\hline & & \\
\hline & & \\
\hline & 0.1 & -0.0 \\
\hline & & \\
\hline & & -0.0 \\
\hline & 0.16 & -0.0 \\
\hline & 0.15488 & -0.0036 \\
\hline
\end{tabular}


$85 \mathrm{H} \quad 0.146283-0.000690$

$\begin{array}{llll}86 \mathrm{H} & 0.139471 & -0.002020\end{array}$

$\begin{array}{lllll}87 \mathrm{H} & 0.131970 & -0.002186\end{array}$

$\begin{array}{llll}88 \mathrm{H} & 0.101541 & 0.000722\end{array}$

$\begin{array}{llll}89 \mathrm{H} & 0.102845 & 0.000581\end{array}$

$90 \mathrm{H} \quad 0.119695-0.000015$

$\begin{array}{llll}91 \mathrm{H} & 0.118035 & 0.000015\end{array}$

$\begin{array}{llll}92 \mathrm{H} & 0.141013 & 0.000110\end{array}$

$\begin{array}{llll}93 \mathrm{H} & 0.122573 & 0.000091\end{array}$

$94 \mathrm{H} \quad 0.119045-0.000023$

$\begin{array}{llll}95 & \mathrm{H} & 0.142262 & -0.000037\end{array}$

$\begin{array}{llll}96 & \mathrm{H} & 0.131306 & 0.000004\end{array}$

$\begin{array}{llll}97 \mathrm{H} & 0.135677 & 0.000009\end{array}$

$\begin{array}{llll}98 & \mathrm{H} & 0.147579 & 0.000036\end{array}$

Sum of Mulliken charges $=2.00000 \quad 0.00000$ 